\title{
Sampling the Radio Transient Universe: Studies of Pulsars and the Search for Extraterrestrial Intelligence
}

\author{
Jayanth Chennamangalam \\ West Virginia University
}

Follow this and additional works at: https://researchrepository.wvu.edu/etd

\section{Recommended Citation}

Chennamangalam, Jayanth, "Sampling the Radio Transient Universe: Studies of Pulsars and the Search for Extraterrestrial Intelligence" (2014). Graduate Theses, Dissertations, and Problem Reports. 202.

https://researchrepository.wvu.edu/etd/202

This Dissertation is protected by copyright and/or related rights. It has been brought to you by the The Research Repository @ WVU with permission from the rights-holder(s). You are free to use this Dissertation in any way that is permitted by the copyright and related rights legislation that applies to your use. For other uses you must obtain permission from the rights-holder(s) directly, unless additional rights are indicated by a Creative Commons license in the record and/ or on the work itself. This Dissertation has been accepted for inclusion in WVU Graduate Theses, Dissertations, and Problem Reports collection by an authorized administrator of The Research Repository @ WVU.

For more information, please contact researchrepository@mail.wvu.edu. 
Sampling the Radio Transient Universe:

Studies of Pulsars and the Search for Extraterrestrial Intelligence

Jayanth Chennamangalam

Dissertation submitted

to the Eberly College of Arts and Sciences

at West Virginia University

in partial fulfillment of the requirements for the degree of

Doctor of Philosophy in Physics

Duncan Lorimer, Ph.D., Chair

Maura McLaughlin, Ph.D.

D. J. Pisano, Ph.D.

Dan Werthimer, M.S.

Kenneth Showalter, Ph.D.

Department of Physics and Astronomy

Morgantown, West Virginia

2014

Keywords: neutron stars, pulsars, extraterrestrial intelligence, instrumentation, statistics

Copyright 2014 Jayanth Chennamangalam 


\begin{abstract}
Sampling the Radio Transient Universe:

Studies of Pulsars and the Search for Extraterrestrial Intelligence
\end{abstract}

Jayanth Chennamangalam

The transient radio universe is a relatively unexplored area of astronomy, offering a variety of phenomena, from solar and Jovian bursts, to flare stars, pulsars, and bursts of Galactic and potentially even cosmological origin. Among these, perhaps the most widely studied radio transients, pulsars are fast-spinning neutron stars that emit radio beams from their magnetic poles. In spite of over 40 years of research on pulsars, we have more questions than answers on these exotic compact objects, chief among them the nature of their emission mechanism. Nevertheless, the wealth of phenomena exhibited by pulsars make them one of the most useful astrophysical tools. With their high densities, pulsars are probes of the nature of ultra-dense matter. Characterized by their high timing stability, pulsars can be used to verify the predictions of general relativity, discover planets around them, study bodies in the solar system, and even serve as an interplanetary (and possibly some day, interstellar) navigation aid. Pulsars are also used to study the nature of the interstellar medium, much like a flashlight illuminating airborne dust in a dark room. Studies of pulsars in the Galactic center can help answer questions about the massive black hole in the region and the star formation history in its vicinity. Millisecond pulsars in globular clusters are long-lived tracers of their progenitors, low-mass X-ray binaries, and can be used to study the dynamical history of those clusters. Another source of interest in radio transient astronomy is the hitherto undetected engineered signal from extraterrestrial intelligence. The Search for Extraterrestrial Intelligence (SETI) is an ongoing attempt at discovering the presence of technological life elsewhere in the Galaxy. In this work, I present my forays into two aspects of the study of the radio transient universe - pulsars and SETI.

Firstly, I describe my work on the luminosity function and population size of pulsars in the globular clusters Terzan 5, 47 Tucanae, and M 28. Applying Bayesian statistics to our data set consisting of the number of detected pulsars, their flux densities, and the amount of diffuse radio emission from the direction of these clusters, we show that the number of potentially observable pulsars in Terzan 5 should be within a 95 per cent credible interval of $147_{-65}^{+112}$. For 47 Tucanae and M 28, our results are $83_{-35}^{+54}$ and $100_{-52}^{+91}$, respectively. We also constrain the luminosity function parameters for the pulsars in these clusters.

The Galactic center pulsar population has been an interesting target for various studies, especially given that only one pulsar has been detected in the region, when we expect hundreds of pulsars to be present. In this work, we use the scattering 
measurements from recent observations of PSR J1745-2900, the Galactic center pulsar, and show that the size of the potentially observable pulsar population has a conservative upper limit of $\sim 200$. We show that the observational results so far are consistent with this number and make predictions for future radio pulsar surveys of the region.

The Versatile GBT Astronomical Spectrometer (VEGAS) is a heterogeneous instrument used mainly for pulsar studies with the Green Bank Telescope. I describe our work on the GPU spectrometer that we developed as part of VEGAS. The GPU code supports a dual-polarization bandwidth of up to $600 \mathrm{MHz}$.

In the field of SETI, I discuss two works. SERENDIP VI is a heterogeneous SETI spectrometer to be installed both at the Green Bank Telescope and at the Arecibo Observatory. In this work, we describe the design of the GPU spectrometer that forms part of SERENDIP VI. In the second work, we speculate on a novel search strategy for SETI, based on the idea that technological civilizations lacking the advancement required to build high-powered beacons may choose to build a modulator situated around a nearby pulsar, depending on whether it is energetically favorable. We discuss observational signatures to search for, using a model of artificially-nulled pulsars. 


\section{Acknowledgements}

This dissertation would not have been possible had it not been for the support of a large number of people, whom I would like to thank here. Firstly, I would like to thank my advisor, Duncan Lorimer, for being a fantastic guide. Dunc has been a very patient and considerate advisor, and provided me with excellent research opportunities and mentoring. I would also like to thank Dan Werthimer for giving me the opportunity to work with the CASPER group at UC Berkeley, and for his guidance. I would like to thank my other committee members as well - D. J. Pisano, Maura McLaughlin, and Ken Showalter - for their advise on various occasions, including their comments on this dissertation.

Special thanks go to my office mates over the past few years, Nipuni, Ben, and Lucas - thanks to you, office life was never boring! Nipuni was especially helpful with research problems that I struggled with on numerous occasions. I would also like to express my gratitude to the current and past members of the astronomy group, including Manjari, Nate, and Priya, for their support at various stages of my PhD. Sanjay, Oshadha, Justin, and Justin also deserve thanks for their support during the coursework era. The department office staff, especially Sherry Puskar, are appreciated for their help with the paperwork that cropped up now and again. My collaborator Ilya Mandel is appreciated for discussions related to the globular cluster paper, and answering questions on Bayesian statistics.

Among the people I interacted with whilst at Berkeley, several people deserve acknowledgments. In addition to help rendered during the VEGAS project, Mark deserves special appreciation for introducing me to the San Francisco Bay Area nightlife, and other interesting things in and around the area. Unfortunately, our foray into the Mumbai nightlife after the Pune CASPER workshop did not quite go as planned, but has provided fodder for beer-time merriment in the years that followed. The debates with him over beer at Jupiter have been very illuminating as well. Andrew has been an excellent collaborator and sounding board. Simon, Glenn, Hong, Nie Jun, and Anish were superb colleagues during the VEGAS days. The members of the SETI and RAL groups at Berkeley have also been instructive to me, especially, Jeff Cobb, Dave MacMahon, and Matt Dexter.

My life in Morgantown would have been boring had it not been for friends Nikola, Tom, and Brian, among others. Nikola and his interest in gastronomy made sure I was well-fed at home, and I enjoyed our discussions on statistics and life in general. I enjoyed my stay at Big Blue, and I thank Jessika and Todd for providing a pleasant home for us. Tom has been a first-class host, and having Cuba Libres with him has become a tradition that goes back to our Arecibo days. Tom and his wonderful family - fiancée Lauren and mother Mary - are much appreciated for their hospitality on innumerable occasions. I would also like to thank Kurian, my old buddy from college, his wonderful wife Haritha, and their family, for their hospitality throughout the past few years, on my many stopovers in the DC area.

The teachers and students of the Chinese courses I took provided me with much-needed distraction from work, and allowed me to exercise parts of my brain that otherwise might have lain unused. Although my Chinese is still far from con- 
versational - which is entirely my fault! - it was a thoroughly enjoyable experience, and I am appreciative of that, and I look forward to continuing my Chinese studies in the future.

My list of people to thank would not be complete without thanking Avinash Deshpande of RRI, who gave me my first chance to work in astronomy, in spite of me having no background in the field. Many of the things I learned during my time at RRI have proven useful in the course of my Ph.D.

Finally, I thank my partner, Geethmala, for inspiring and supporting me throughout the past few years. I also thank my parents, especially my mother, who cultivated my childhood scientific interests by buying me science encyclopedias that I read voraciously. 


\section{Table of Contents}

List of Tables viii

List of Figures $\quad$ ix

1 Introduction 1

1.1 The Radio Transient Universe . . . . . . . . . . . . . . . . . . . 1

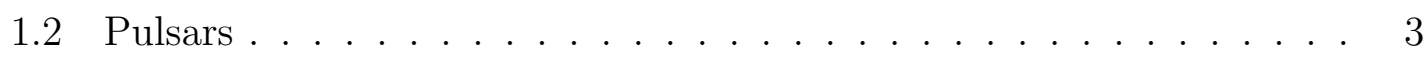

1.2.1 Observing Pulsars . . . . . . . . . . . . . . . . . 8

1.2.2 Pulsar Population Statistics . . . . . . . . . . . . . . 10

1.2.3 Instrumentation for Pulsar Studies . . . . . . . . . . . . . 17

1.3 SETI . . . . . . . . . . . . . . . . . . . . 19

1.3.1 Detection Considerations . . . . . . . . . . . . 20

1.3.2 SETI Instrumentation . . . . . . . . . . . . . . . 21

1.4 Dissertation Outline . . . . . . . . . . . . . . . 22

2 Constraining the luminosity function parameters and population size of radio

$\begin{array}{ll}\text { pulsars in globular clusters } & 23\end{array}$

2.1 Introduction . . . . . . . . . . . . . . . . . 23

2.2 Bayesian parameter estimation . . . . . . . . . . . . . . . . 26

2.2.1 Using pulsar flux densities . . . . . . . . . . . . . 30

2.2.2 Incorporating the number of observed pulsars . . . . . . . . 30

2.2.3 Considering diffuse emission . . . . . . . . . . . . . . . 31

2.2.4 Transformation to luminosity domain . . . . . . . . . . 33 
2.3 Applications . . . . . . . . . . . . . . . . . 34

2.3 .1 Using prior information . . . . . . . . . . . . . . . 41

2.4 Discussion . . . . . . . . . . . . . . . . 43

2.4.1 Effect of flux density measurement errors on credible intervals 43

2.4.2 Effect of increasing detections on credible intervals . . . . . . 44

2.5 Summary and conclusions $\ldots \ldots \ldots \ldots \ldots$

3 The Galactic center pulsar population $\quad 48$

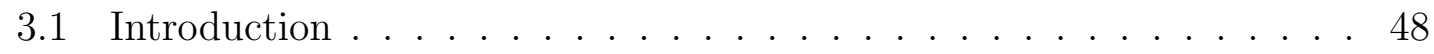

3.2 Constraining the GC pulsar content . . . . . . . . . . 50

3.2 .1 Bayesian Approach . . . . . . . . . . . . . . . . 50

3.2 .2 Monte Carlo Approach . . . . . . . . . . . . . . . . 56

3.3 Discussion . . . . . . . . . . . . . . . . 60

4 A GPU-Based Wide-Band Radio Spectrometer 65

4.1 Introduction . . . . . . . . . . . . . 65

4.2 The GPU-Programming Paradigm . . . . . . . . . . . . . 67

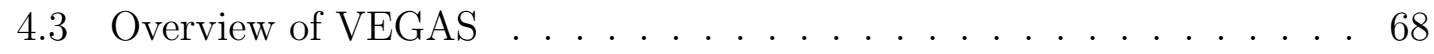

4.4 The GPU Spectrometer $\ldots \ldots \ldots \ldots \ldots$

4.4.1 The Spectrometer Algorithm . . . . . . . . . . . . . . . . 72

4.5 Benchmarking and Performance Results . . . . . . . . . . 74

4.6 Conclusions . . . . . . . . . . . . . . . . . 75

5 SERENDIP VI 76 


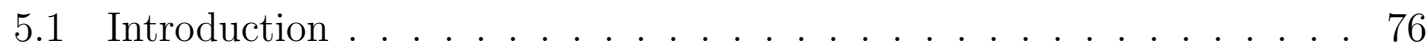

5.2 System Architecture . . . . . . . . . . . . . . . . . 77

$5.3 \quad$ FPGA Design $\ldots \ldots \ldots \ldots \ldots \ldots \ldots \ldots \ldots \ldots$

5.4 Data Transport Framework _. . . . . . . . . . . . . . 81

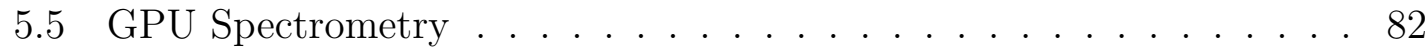

5.5.0.1 Handling Long Transforms . . . . . . . . . . . . . . 83

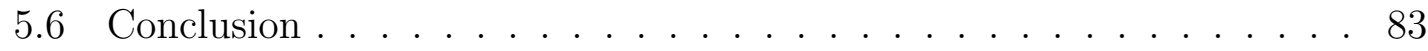

6 Jumping the energetics queue: Modulation of pulsar signals by extraterrestrial civilizations

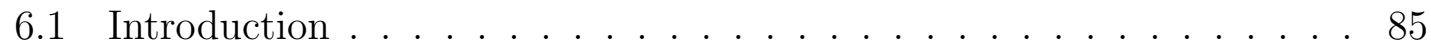

6.2 Modulation mechanism . . . . . . . . . . . . . . . . . . . . 89

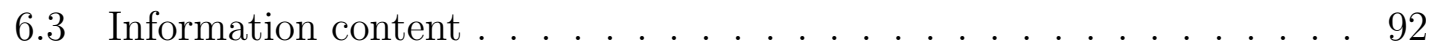

6.4 Observable effects . . . . . . . . . . . . . . . . . . 93

6.5 Discussion . . . . . . . . . . . . . . 97

6.6 Conclusion . . . . . . . . . . . . . . . . . . 99

$\begin{array}{lll}7 & \text { Conclusion } & 100\end{array}$

7.1 Instrumentation for transient search/studies . . . . . . . . 100

7.2 Population statistics of globular cluster pulsars . . . . . . . . . . 101

7.3 The Galactic center pulsar population . . . . . . . . . . . . . . . 101

7.4 SETI . . . . . . . . . . . . . . . . . 102 


\section{List of Tables}

2.1 Flux densities used in the analysis. . . . . . . . . . . . . 36

2.2 Median and credible intervals. . . . . . . . . . . . . . . . 43

3.1 Results of Bayesian analysis for a few GC surveys. . . . . . . . . . 56

3.2 Predictions for surveys, both past and future. . . . . . . . . 63

4.1 VEGAS heterogeneous modes . . . . . . . . . . . 68 


\section{List of Figures}

1.1 Emission geometry of a pulsar. . . . . . . . . . . . . . . 4

$1.2 P-\dot{P}$ diagram. . . . . . . . . . . . . . . . . . . 12

1.3 Pulsar positions - Aitoff projection. . . . . . . . . . . . . . 14

1.4 Pulsar positions - top-view of the Galaxy. . . . . . . . . . . . . 15

1.5 Schematic of a typical pulsar back-end. . . . . . . . . . . . . . 18

2.1 Logical flow of the Bayesian analysis . . . . . . . . . . . . . . 29

2.2 Results of the Bayesian analysis for Terzan 5 . . . . . . . . . . . 40

2.3 Posteriors on $N$ after applying priors on $\mu$ and $\sigma \quad \ldots . . . . . . \quad 42$

2.4 Results of the Monte Carlo simulation described in $§ 2.4 .2 . \quad$. . . . . 45

3.1 Posterior probability density functions of $N \ldots \ldots \ldots 5$

3.2 The 99 per cent upper limit on $N$ as a function of magnetar fraction. $\quad 57$

$3.3 n_{\mathrm{obs}}$ versus $\langle N\rangle$ and $\left\langle N_{\mathrm{GC}}\right\rangle \ldots \ldots \ldots \ldots \ldots$

4.1 Data flow diagram of the VEGAS software pipeline. . . . . . . . . 69

4.2 Single-bin frequency response comparison of PFB vs. direct FFT. . . 71

4.3 GPU spectrometry flowchart. . . . . . . . . . . . . 73

4.4 GPU spectrometer performance. . . . . . . . . . . . . 75

5.1 SERENDIP VI system architecture. . . . . . . . . . . . . 79

5.2 SERENDIP VI FPGA design. . . . . . . . . . . . . . . . 80

5.3 SERENDIP VI UDP packet payload format. . . . . . . . . . . . . 80

5.4 SERENDIP VI data transport framework. . . . . . . . . . . . . . 82 
5.5 Spectra from simulated data. . . . . . . . . . . . . . . . 84

6.1 Pulsar modulation schematics. . . . . . . . . . . . . . . . 90

6.2 Example of a non-natural nulling process. . . . . . . . . . . . . . 95 


\section{List of Abbreviations and Symbols}

\begin{tabular}{|c|c|}
\hline $\mathrm{ADC}$ & Analog to digital converter \\
\hline ATNF & Australia Telescope National Facility \\
\hline CASPER & $\begin{array}{l}\text { Collaboration for Astronomy Signal Processing and Electronics Re- } \\
\text { search }\end{array}$ \\
\hline CUDA & Compute Unified Device Architecture \\
\hline DFT & Discrete Fourier Transform \\
\hline ETI & Extraterrestrial intelligence \\
\hline FFT & Fast Fourier Transform \\
\hline FPGA & Field programmable gate array \\
\hline FRB & Fast radio burst \\
\hline GBT & Green Bank Telescope \\
\hline $\mathrm{GC}$ & Globular cluster or Galactic center, depending on the context \\
\hline GPU & Graphics processing unit \\
\hline $\mathrm{HPC}$ & High Performance Computing \\
\hline ISM & Interstellar medium \\
\hline LMC & Large Magellanic Cloud \\
\hline LMXB & Low-mass X-ray binary \\
\hline MSP & Millisecond pulsar \\
\hline PFB & Polyphase filter bank \\
\hline RAM & Random Access Memory \\
\hline $\mathrm{ROACH}$ & Reconfigurable Open Architecture Computing Hardware \\
\hline RRAT & Rotating radio transient \\
\hline SERENDIP & $\begin{array}{l}\text { Search for Extraterrestrial Radio Emissions from Nearby Developed } \\
\text { Intelligent Populations }\end{array}$ \\
\hline SETI & Search for Extraterrestrial Intelligence \\
\hline $\mathrm{SMC}$ & Small Magellanic Cloud \\
\hline SNR & Supernova remnant \\
\hline UDP & User Datagram Protocol \\
\hline VEGAS & Versatile GBT Astronomical Spectrometer \\
\hline$B$ & Surface magnetic field \\
\hline$c$ & Velocity of light in vacuum \\
\hline$\dot{E}$ & Spin-down luminosity \\
\hline$I$ & Moment of inertia \\
\hline$k$ & Boltzmann constant \\
\hline$M_{\odot}$ & Solar mass \\
\hline$P$ & Pulsar spin period \\
\hline$\dot{P}$ & First derivative of pulsar spin period \\
\hline$S / N$ & Signal-to-noise ratio \\
\hline$T_{B}$ & Brightness temperature \\
\hline
\end{tabular}




\section{Chapter 1}

\section{Introduction}

\subsection{The Radio Transient Universe}

Time domain astronomy - the study of time-dependent variability in what at first glance seems to be a static or slowly-changing universe - is an important field in astronomy. Radio astronomy has a long history of time domain work, thanks to the discovery of radio pulsations from what came to be known as pulsars - rapidly spinning, highly magnetized neutron stars (Hewish et al., 1968, see also §1.2). But pulsars are not alone in the class of 'fast' (i.e., short-duration, time scale $\lesssim 10$ s) radio transients ${ }^{1}$. Fast radio transients include, in addition to regular pulsars, Rotating Radio Transients (RRATs; McLaughlin et al., 2006) solar bursts (Ellis \& McCulloch, 1967), Jovian bursts (for a recent example, see Arkhypov \& Rucker, 2009), flare stars (Lang et al., 1983), and the recently confirmed class of sources known as Fast Radio Bursts (FRBs; Lorimer et al., 2007; Thornton et al., 2013). In addition to these known fast transients, more have been speculated, including prompt emission from supernovae (Colgate \& Noerdlinger, 1971), exploding black holes (Rees, 1977), beacons from intelligent civilizations elsewhere in the Galaxy (see, for example, Siemion et al., 2013a), etc. For an overview of the techniques involved in searching for fast radio transients, see Cordes \& McLaughlin (2003).

\footnotetext{
'Slow' (i.e., long-duration) transients such as supernova light-curves are beyond the scope of this dissertation.
} 
Many kinds of fast radio transients are interesting because they are indicators of energetic processes associated with compact objects. Whereas the origin of most fast radio transients are understood to some extent, the recently confirmed class of sources known as FRBs is still a mystery. Unlike the other transients that originate in the Galaxy or in nearby satellite galaxies, FRBs are short-duration, bright radio bursts believed to originate at cosmological distances. Proposed progenitors include flaring magnetars (Popov \& Postnov, 2013), binary neutron star mergers (Totani, 2013), and gravitational collapse of neutron stars to black holes (Falcke \& Rezzolla, 2013). There is at least one Galactic explanation for FRBs, in the form of nearby flare stars (Loeb et al., 2014). In any case, it is clear that searching for fast radio transients opens up possibilities for serendipitous discoveries, and studying such phenomena has the potential to shed light on extreme physics in action.

One of the less conventional searches that astronomers undertake is that for Extraterrestrial Intelligence (ETI). The Search for Extraterrestrial Intelligence (SETI) is a loose collection of different kinds of searches that have been undertaken to detect signs of intelligent technological life elsewhere in the Galaxy. While such efforts have sampled all over the electromagnetic spectrum, from radio (Siemion et al., 2013a, for instance) to infrared (Carrigan, Jr., 2009, for instance), the major band of interest to SETI is the radio. An unambiguous detection of an ETI signal will change not just the field of astrobiology, but has the potential to affect human society profoundly.

This dissertation deals with two categories of radio transients, namely, pulsars and ETI. In the following chapters, I describe my work on pulsar instrumenta- 
tion and pulsar population statistics, and SETI instrumentation and a novel SETI search strategy, involving pulsars. This chapter introduces the relevant background required for the remainder of this dissertation.

\subsection{Pulsars}

A pulsar is a neutron star that has a high magnetic field, rotates rapidly, and emits electromagnetic radiation, most prominently in a beamed fashion, directed outward away from its two magnetic poles (see Figure 1.1). Pulsars are most commonly detected by virtue of the fact that their rotational axes and magnetic dipolar axes are not necessarily aligned. This leads to the radio emission of the pulsar to appear as periodic pulses to a distant, stationary (within limits) observer as the emission beam crosses the line of sight, with period equal to the spin period of the pulsar. This is akin to what one experiences from a lighthouse, where a pulse of light is observed when the light beam from the rotating lens assembly crosses one's line of sight. The emitted radiation is usually in the radio region of the electromagnetic spectrum, although there exist pulsars that emit at higher frequencies, including optical, X-rays, and gamma rays. Pulsars that emit in the radio are known as 'radio pulsars', and are the most common kind of pulsars. In fact, the very first pulsar discovered was detected in the radio (Hewish et al., 1968). Since then, about 2300 pulsars have been discovered ${ }^{2}$.

Radio pulsars have spin periods ranging from about $1.4 \mathrm{~ms}$ (Hessels et al.,

\footnotetext{
${ }^{2}$ As per the ATNF Pulsar Catalogue v1.48 (as of November 2013; Manchester et al., 2005): http://www .atnf.csiro.au/people/pulsar/psrcat
} 


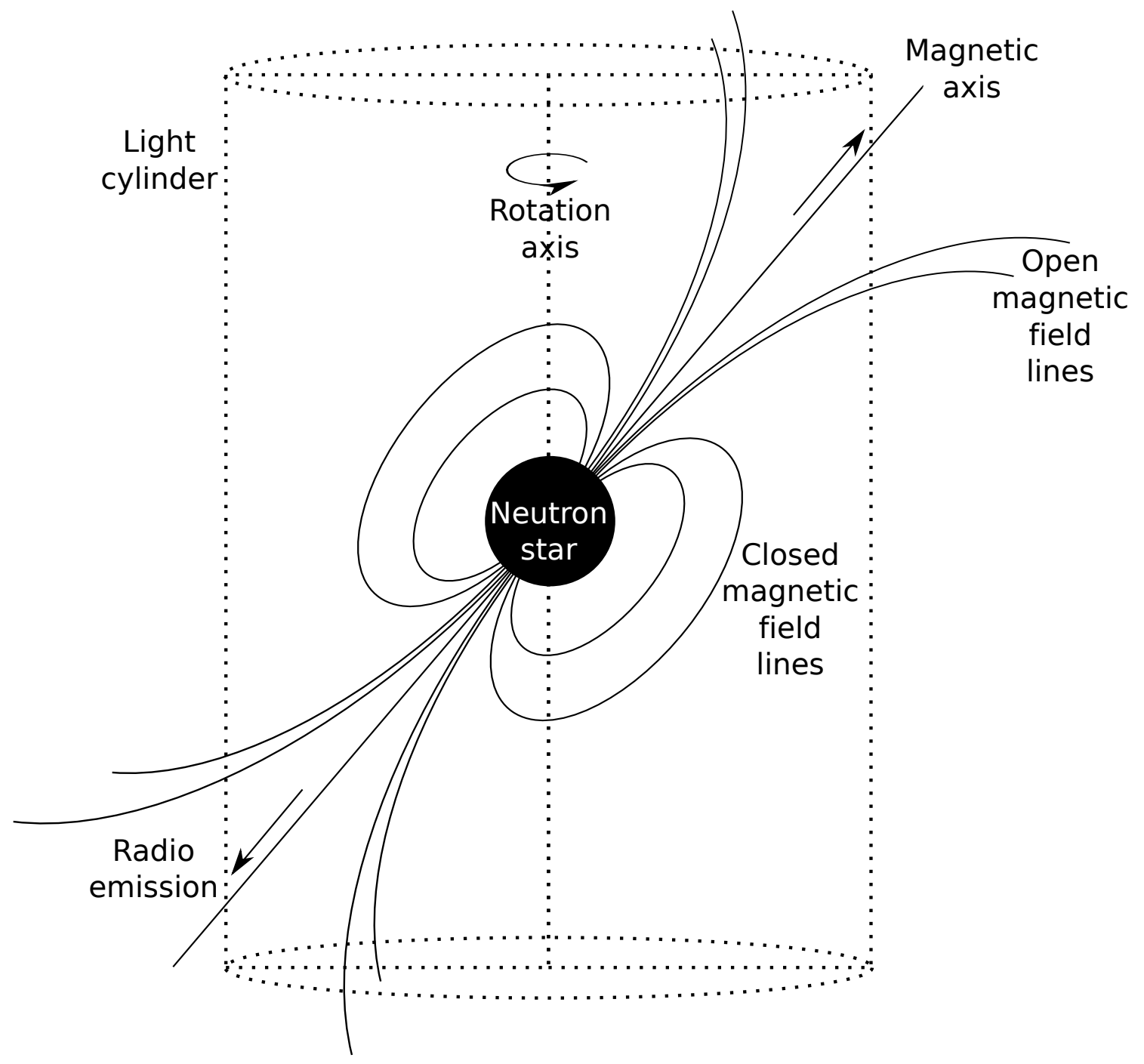

Figure 1.1: Emission geometry of a pulsar. 
2006) to about $8.5 \mathrm{~s}$ (Young et al., 1999), and duty cycles $^{3}$ in the range 0.6 per cent to 79 per cent, with a median duty cycle of around 5 per cent. Light-traveltime arguments, together with the short periodicity that intuitively suggests fast rotation, imply that the source of such emission must be of small physical size. Pulsars are therefore understood to be compact objects. The luminosity of their thermal emission, where observable, can be used to infer the size of the emission region, and hence their radii (see, for example, Golden \& Shearer, 1999; Lattimer \& Prakash, 2001), with a typical radius of $10 \mathrm{~km}$. Studies of pulsars in binary systems have shown that their masses are around $1.4 M_{\odot}$, with the most massive known pulsars having a mass of $\sim 2 M_{\odot}$ (Demorest et al., 2010; Antoniadis et al., 2013), showing that these are highly dense objects. The central density of pulsars reach $\sim 10^{15} \mathrm{~g} \mathrm{~cm}^{-3}$, at which point matter can only exist in the form of degenerate neutrons. In other words, pulsars are neutron stars.

Pulsars originate in supernova explosions, and are the remnant core of massive stars $\left(\sim 8 M_{\odot}-20 M_{\odot}\right)$. They have a high surface magnetic field, which is due to the conservation of magnetic flux during core collapse. The high spin rate is due to the conservation of angular momentum during core collapse. The rotation of these massive stars is quite stable, which leads to the stability in the timing of their pulses. Pulsars do slow down, however, by a tiny amount. The first derivate of the pulse period, or the pulsar's 'spin-down rate', $\dot{P}$, can be as low as $\sim 10^{-21} \mathrm{~s} \mathrm{~s}^{-1}$. In fact, it is this spin-down that is the source of the energy radiated by the pulsar in

\footnotetext{
${ }^{3}$ The pulsar duty cycle, $\delta=W_{\text {eq }} / P$, where $W_{\text {eq }}$ is the equivalent pulse width and $P$ is the spin period.
} 
the radio. That is, radio pulsars are 'rotation-powered'. It can be shown that the energy loss of a pulsar due to its spin-down - known as the 'spin-down luminosity' - is given by

$$
\dot{E}=4 \pi^{2} I \dot{P} P^{-3}
$$

where $I$ is the moment of inertia of the star, $P$ is the spin period of the pulsar, and $\dot{P}$ is the first derivative of the spin period. For the canonical value of $I$ of $10^{45} \mathrm{~g}$ $\mathrm{cm}^{2}$

$$
\dot{E} \approx 3.95 \times 10^{31}\left(\frac{\dot{P}}{10^{-15}}\right)\left(\frac{P}{\mathrm{~s}}\right)^{-3} .
$$

Of this spin-down energy loss, only a small fraction appears as radio emission, with the rest dissipated through other mechanisms, such as magnetic dipole radiation and the pulsar wind.

The brightness temperature of radio pulsars - i.e, the equivalent temperature of a blackbody with the same observed intensity at a given frequency - can be calculated in the Rayleigh-Jeans regime as

$$
T_{B}=\frac{I_{\nu} c^{2}}{2 k \nu^{2}}
$$

where $I_{\nu}$ is the intensity of radio emission, $c$ is the velocity of light in vacuum, $k$ is the Boltzmann constant, and $\nu$ is the frequency of observation. The intensity of the radio emission, or the luminosity of a pulsar with spin period $P$ situated at a 
distance $d$ from an observer is given in terms of the measured flux density as

$$
L=\frac{4 \pi d^{2}}{\delta} \sin ^{2}\left(\frac{\rho}{2}\right) \int_{\nu_{1}}^{\nu_{2}} S_{\text {mean }}(\nu) d \nu
$$

where $\delta$ is the pulse duty cycle, $\rho$ is the radius of the pulsar emission cone, the integrand is the mean flux density of the pulsar as a function of frequency $\nu$, and $\nu_{1}$ and $\nu_{2}$ are the bounds of the spectral range of observation. Using the median values of measured flux densities and distances for pulsars in the ATNF Pulsar Catalogue, it can be shown that $T_{B}$ is on the order of $10^{24} \mathrm{~K}$. The high brightness temperature of pulsars is thought to imply a coherent mechanism (see, for example, Gold, 1968), although the validity of that conclusion has been examined by Zhang, Hong \& Qiao (1999), who conclude that coherence may not be essential for pulsar radio emission, and that mechanisms such as inverse Compton scattering may be invoked to explain the emission.

The exact mechanism behind the observed emission of pulsars is poorly understood. The most commonly used model is due to Goldreich \& Julian (1969), wherein the authors show that the electric field just above the surface of the neutron star is strong enough to pull charged particles out of the surface charge layer, forming a magnetosphere around the star. The charged particles so extracted, follow the magnetic field lines that also turn out to be electric equipotentials. Closer to the equator of the neutron star, the magnetic field lines are closed, i.e., they reconnect with the surface of the star. Closer to the poles, however, the magnetic field lines are open, and the plasma streams out along these lines, forming a 'pulsar wind'. The 
charged particles in the magnetosphere are forced to co-rotate with the pulsar, and this co-rotation can be maintained only up to what is known as the 'light cylinder', beyond which particles would have to have superluminal velocities to keep up with the rotation. The locations of the last open field lines define the polar cap of the pulsar (see Figure 1.1).

In spite of over 40 years of research, pulsar astronomy remains largely phenomenological in nature. The physical mechanism of pulsar emission, the true nature of the stellar magnetosphere, and the structure of the pulsar itself are not well understood. Despite this, however, the pulsar phenomenology is so rich and varied that pulsars are used as a very useful tool in dealing with problems as diverse as the equation of state of dense matter, validity of general relativity, the nature of the interstellar medium (ISM), studies of the solar system, planet formation, and even spacecraft navigation.

\subsubsection{Observing Pulsars}

Radio pulsars are broad-band emitters, with most pulsars being visible from an observing frequency of a few 10 s of $\mathrm{MHz}$ to a few $\mathrm{GHz}$. The lower limit is due to ISM effects and the opacity of the Earth's atmosphere at low frequencies, and the upper limit is mainly due to the steep spectral index characteristic of pulsars, that causes a decrease of luminosity with increasing frequency. The spectral index has a power-law form, with the measured flux density $S(\nu) \propto \nu^{\alpha}$, where $\nu$ is the observing frequency and $\alpha$ is the spectral index. The spectral index is not the same for all 
pulsars, but can be modelled as a Gaussian distribution with a mean of -1.4 and a standard deviation of 1 (Bates et al., 2013). The steep spectral index is thought to be another indicator that the pulsar emission is due to a coherent mechanism, as the coherence volume decreases with increasing frequency (decreasing wavelength).

Pulsar observations are affected by the properties of the ISM. The ISM is made up of neutral and ionized gases, both diffuse (as part of the Galactic disk), and clumpy (molecular clouds, supernova remnants, etc.). For the purpose of simplicity and ease of modelling, in pulsar astronomy, the ISM is usually taken to be completely ionized, and made up of just electrons. The column density of electrons in the line of sight towards a pulsar determines how the pulsar signal is received by the observer. The most striking effect of the ISM on the pulsations is that the broad-band signal is dispersed, following a quadratic law. That is, the lower frequencies are progressively delayed compared to the higher frequencies. This means that if one were to observe a pulsar with an instrument that only computed the total power received at each instant of time, the pulsar's pulses would be observed to be much broader than its intrinsic width and weaker than expected, and, depending on the distance travelled by the signal, can be completely smeared out. It is necessary, therefore, to correct for this ISM effect, using a process known as 'dedispersion'. To dedisperse a signal, the signal is Fourier transformed to multiple channels, such that the dispersion within each channel is negligible. This setup is called a 'filterbank'.

The ISM causes another effect on the pulsar signal, namely, scattering. Scattering, which originates due to inhomogeneities in the ISM, follows a $\nu^{-4}$ law, wherein the lower frequencies are much more scattered than the higher frequen- 
cies. This reduces the signal-to-noise ratio $(S / N)$ at low frequencies, so, in spite of the fact that pulsars are intrinsically brighter at those frequencies, depending on the distance, or the density of electrons in their direction, they may be rendered undetectable. In fact, scattering may be a reason for the paucity of detections in the high-electron-density Galactic Center region (Cordes \& Lazio, 1997).

Another ISM effect of importance to pulsar observations is that due to the turbulence in the inhomogeneous ISM, namely, interstellar scintillation. Scintillation serves to either brighten or dim the pulsar signal. This is also a time-dependent effect. Scintillation can make previously undetected pulsars detectable, but can also render previously detected pulsars undetectable. The observed flux density of pulsars varies due to scintillation, which makes determining their luminosities difficult. An extreme example is the case of 47 Tucanae, a globular cluster that harbours 23 pulsars, in which most pulsars remain undetectable on most days (Camilo et al., 2000).

\subsubsection{Pulsar Population Statistics}

Statistical studies of the ensemble of pulsars in the Galaxy offer insights into their formation mechanisms, time evolution, emission mechanisms, and also, the true size of the potentially observable population. Faucher-Giguère \& Kaspi (2006) have estimated that there are $\gtrsim 120,000$ potentially observable pulsars in the Galaxy, indicating that, in spite of the large number of detections so far, we have only sampled the tip of the iceberg. 
There are three distinct populations of radio pulsars, differentiated based on their periodicity and surface magnetic field strength - 'normal' pulsars, 'recycled' pulsars, and 'magnetars'. The first, and most populous category is made up of pulsars with periods greater than $\sim 30 \mathrm{~ms}$, and having a typical surface magnetic field strength of $10^{12}$ G. Recycled pulsars, also known as 'millisecond pulsars' ('MSPs'), have periods $\lesssim 30 \mathrm{~ms}$, and have typical magnetic field strengths of $10^{8} \mathrm{G}$. Magnetars are pulsars with periods in the range $\sim 2-12 \mathrm{~s}$ and a surface magnetic field strength on the order of $10^{14} \mathrm{G}$. Normal pulsars are young pulsars with a typical characteristic age of $10^{7}$ yr. MSPs, on the other hand, have typical characteristic ages of $10^{9} \mathrm{yr}$ and are believed to have been spun up, or recycled, by accretion from a companion in a binary system (Alpar et al., 1982; Archibald et al., 2009). Indeed, around 80 per cent of all MSPs are in binary systems, when only about 1 per cent of normal pulsars are found in binaries. Figure 1.2 shows a scatter plot of pulsar period and period derivate, and the two populations of normal pulsars and MSPs are readily distinguishable.

Magnetars are the most mysterious of pulsars, and as yet, it is unclear what stage of the evolution of pulsars magnetars occupy. In addition to their unusually high magnetic fields, magnetars are also characterized by a flat spectral index, suggesting that the emission mechanism of magnetars and pulsars may not be the same, and perhaps not coherent at all. Magnetars are also the rarest breed of pulsars, with only 26 known so far ${ }^{4}$.

\footnotetext{
${ }^{4}$ As of February 2014, per the McGill Magnetar Catalog: http://www.physics.mcgill.ca/ $\sim$ pulsar/magnetar/main.html
} 


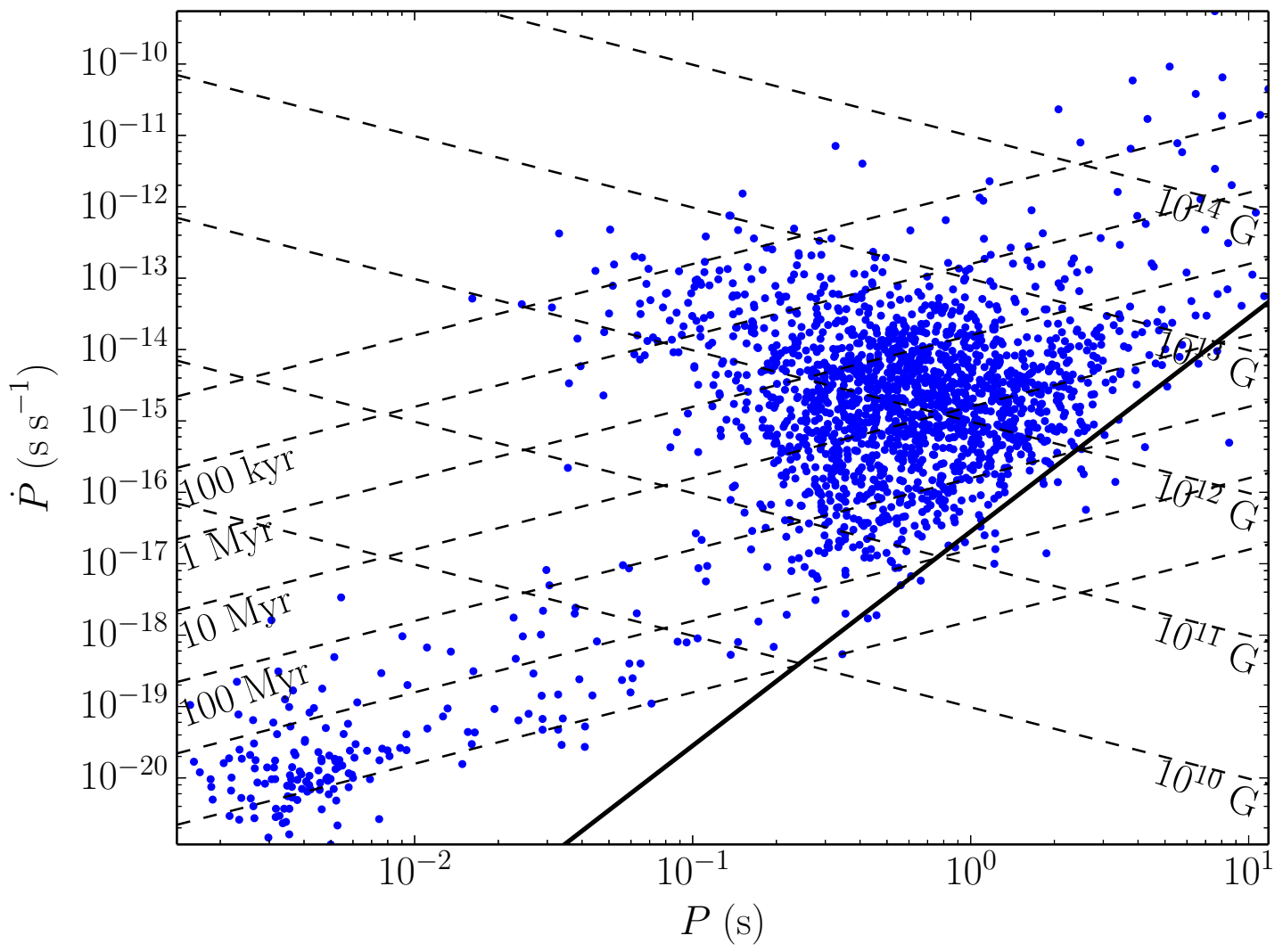

Figure 1.2: A scatter plot of pulsar period versus first derivative of the period, popularly known as the ' $P-\dot{P}$ diagram', for 2005 of the known pulsars. The big cluster is mostly made up of normal pulsars, while the smaller cluster in the lower left are the MSPs. Lines of constant surface magnetic field and constant characteristic age are also shown. The dark line that runs from the bottom left to the top right is the so-called 'death line', to the bottom right of which pulsars do not emit in the radio. Normal pulsars evolve from the top left to the bottom right of the plot, cross the death line, and become radio quiet. A fraction of these pulsars that happens to be in binary systems gets recycled, and turns up at a later time in the MSP cluster. 
Pulsar number density as a function of location typically follows the stellar number density, and as expected, pulsars are mostly found in the disk of the Galaxy. Figure 1.3 shows an Aitoff projection of the locations of most pulsars in the ATNF Pulsar Catalogue, and the Galactic disk traced out by these objects can be clearly seen. Many pulsars outside the Galactic disk - most of them MSPs - are found in globular clusters, while others have been kicked away from the disk during their progenitor supernovae. Pulsars have also been discovered in the Large Magellanic Cloud (LMC) and the Small Magellanic Cloud (SMC), and these are visible as the small clusters in the bottom left of the figure. Many pulsars, especially disk pulsars, are associated with supernova remnants (SNRs), lending credence to the supernova origin of these objects. Figure 1.4 shows a top view of the Galaxy, with the locations of the pulsars inferred based on the NE2001 model of electron density in the Galaxy (Cordes \& Lazio, 2002b). As can be seen, the observed sample is heavily biased in favour of the nearest pulsars, with only a few bright pulsars detected from farther away.

The large number of detected pulsars makes this class of objects suitable for a wide range of statistical analyses. One of the major fields of study of the pulsar population in the Galaxy relates to its luminosity distribution. The luminosity distribution of pulsars is numerically computed usually using one of two methods - a dynamical method, and a snapshot method (see Bagchi et al., 2011, for a brief description of the two methods). Faucher-Giguère \& Kaspi (2006) have performed a full dynamical Monte Carlo simulation of the normal pulsar population in the Galaxy, and have shown that the luminosity distribution of these pulsars follows a 


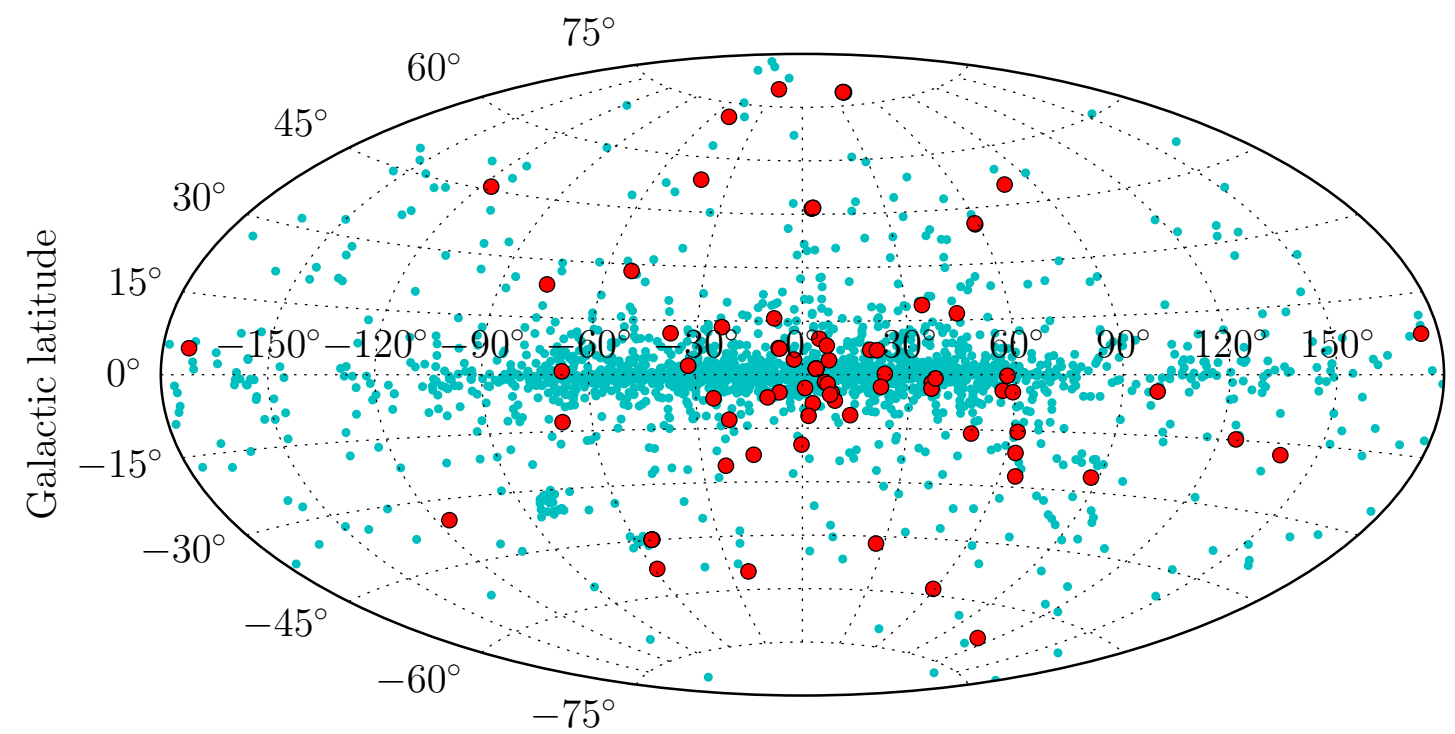

Galactic longitude

Figure 1.3: The locations of known pulsars shown in an Aitoff projection. As can be seen, the number density of pulsars is greatest in the disk of the Galaxy. The clusters in the lower left of the figure are the Large Magellanic Cloud and the Small Magellanic Cloud. The cyan dots are normal pulsars and the red circles are MSPs. It is evident that the locations of MSPs do not follow the structure of the Galactic disk. Indeed, most MSPs reside outside the disk, in globular clusters. 


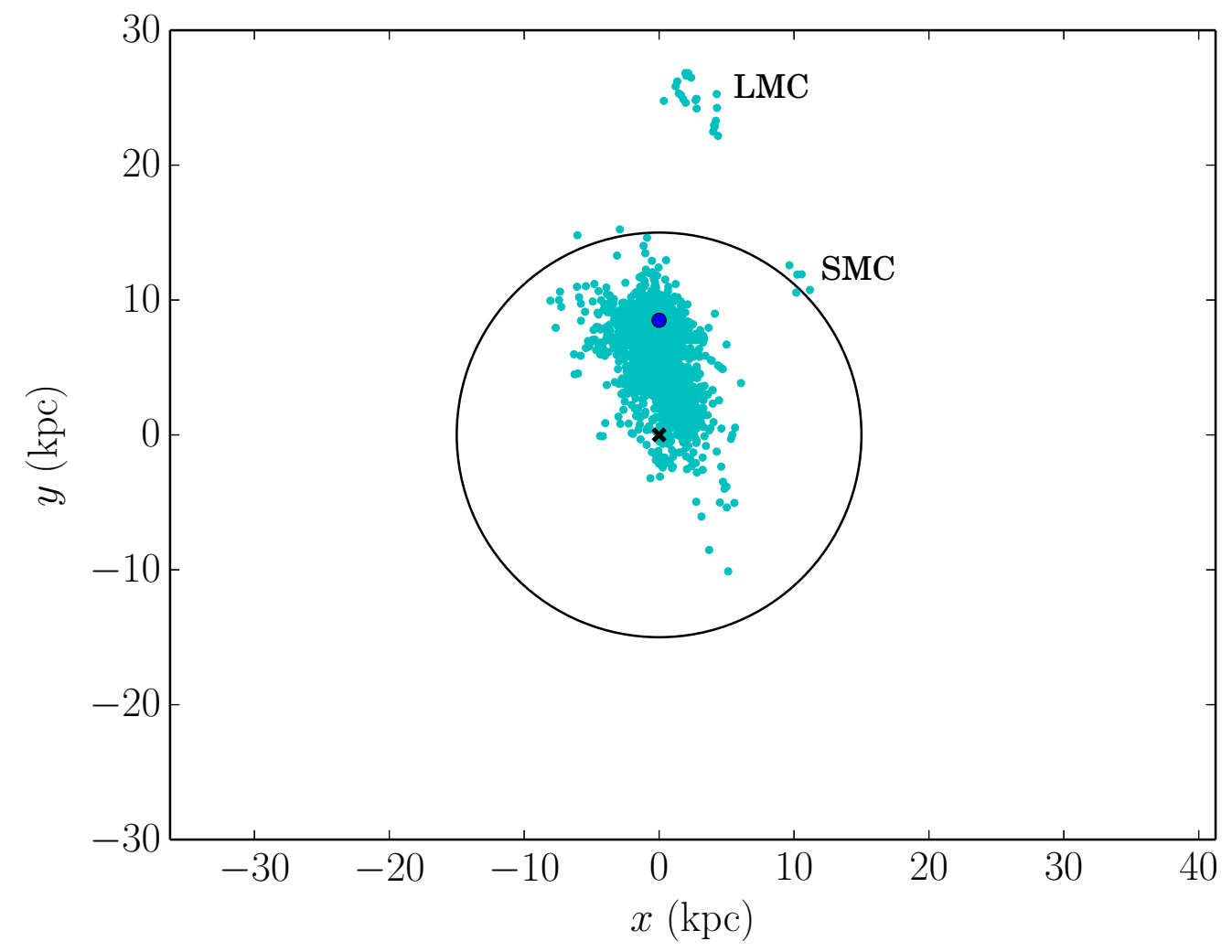

Figure 1.4: The locations of 2213 pulsars (cyan dots) in a top view of the Galaxy and its surroundings, using distance estimates based on the NE2001 model of Galactic electron density (Cordes \& Lazio, 2002b). The black cross indicates the Galactic Center, and the circle, of radius $15 \mathrm{kpc}$, represents the extent of the Galaxy. The blue circle indicates the location of the Earth. The observational bias is readily apparent, with detections clustered around Earth. The Magellanic Clouds can also be seen. Note that this is a two-dimensional projection, and is the reason the SMC appears to be at the edge of the Galaxy. 
log-normal (base-10), with parameters $\mu=-1.1$ and $\sigma=0.9$.

Pulsar population statistics can also be applied to certain regions of the Galaxy. Two regions of interest are globular clusters and the Galactic Center. Globular clusters are spherical agglomerations of stars located throughout the haloes of galaxies. Once thought to be composed entirely of old metal-poor population II stars, they are now also believed to form during interactions or collisions of galaxies, therefore containing younger stars having higher metallicities (see Zepf, 2003). The core stellar number density in globular clusters reach $10^{6} \mathrm{pc}^{-3}$, and such high densities lead to dynamical interactions between stellar systems that are not found commonly in the Galactic plane. For example, globular clusters favour the formation of low-mass X-ray binaries (LMXBs) that are believed to be the progenitors of MSPs, and hence, the fraction of MSPs among all pulsars in globular clusters is much larger than that in the Galactic field ( $\sim 97 \%$ versus $\sim 11 \%$ ). In addition, the binary MSPs in globular clusters tend to have higher eccentricities compared to their field counterparts, due to exchange or fly-by encounters. MSPs, due to their formation history, can be considered long-lived tracers of LMXBs, and therefore, constraints on the MSP content of globular clusters provide unique insights into binary evolution and the integrated dynamical history of globular clusters, while determining the radio luminosity function of these pulsars helps shed light on the radio emission mechanism in action in these compact objects. Pulsar searches of globular clusters have yielded impressive returns in recent years (see Camilo \& Rasio, 2005), with currently 144 pulsars known in 28 clusters $^{5}$. This enables us to

\footnotetext{
${ }^{5}$ See Paulo Freire's globular cluster pulsar catalogue at http://www.naic.edu/ pfreire/
} 
perform statistical studies about the luminosity function, as well as to estimate the total number of pulsars in globular clusters.

The Galactic Center is another region of interest in pulsar astronomy, but for the opposite reason - in spite of decades of observations of the region, only one pulsar has been discovered. Discovering radio pulsars in the Galactic Center holds the promise of probing the gravitational field of the massive black hole in the region (e.g., Pfahl \& Loeb, 2004; Liu et al., 2012), and in deciphering the nature of the interstellar medium in its vicinity. The only pulsar in the inner parsec of the Galactic Center is an $\sim 3.76$ s magnetar (Kennea et al., 2013; Mori et al., 2013; Eatough et al., 2013b; Buttu et al., 2013), located 2.4" from Sgr A* (Rea et al., 2013). At the GC distance of $8.25 \mathrm{kpc}$ (Genzel et al., 2010), this corresponds to a minimum distance of $\sim 0.1 \mathrm{pc}$. Interstellar scattering was thought to be a major problem in detecting pulsars in the Galactic Center, with a predicted scattering timescale of up to $1240 \mathrm{~s}$ at $1 \mathrm{GHz}$. However, Spitler et al. (2013) have measured the scattering in the direction of the magnetar to be $\sim 1.3 \mathrm{~s}$ at $1 \mathrm{GHz}$, much less severe than anticipated. Even though observations of the region have revealed only one pulsar so far, that, along with the scattering measurement allows us to place constraints on the observable pulsar content in the region (see Chapter 3).

\subsubsection{Instrumentation for Pulsar Studies}

Radio pulsars, being fast transients, require special instrumentation for observation.

These 'pulsar back-ends' have high time resolution (typically a few 10s of $\mu$ s), and GCpsr.html 


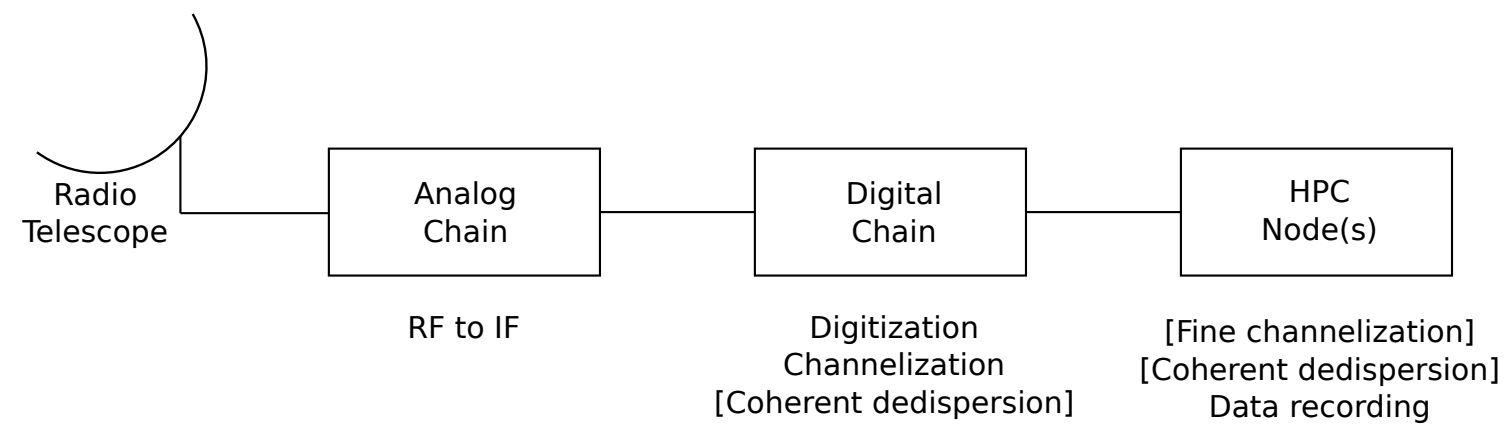

Figure 1.5: Schematic of a typical pulsar back-end. The operations in brackets are optional.

large bandwidths (a few hundred $\mathrm{MHz}$ to a few $\mathrm{GHz}$ ). The former is required to resolve the pulses, especially of MSPs, while the latter is typically required to increase the $S / N$. The schematic of a typical digital pulsar back-end is shown in Figure 1.5. The radio frequency (RF) signal for each polarization is converted to an intermediate frequency (IF) signal in the analog part of the receiver chain. In the digital part of the chain, the IF signals are digitized using fast-sampling Analog to Digital Converters (ADCs), and fed to a signal processing board equipped with a Field Programmable Gate Array (FPGA) chip. The FPGA performs the preliminary signal processing, primarily, channelization, and in some cases, online, coherent dedispersion (for known pulsars). The channelized data are then sent to one or more data processing and recording high-performance computing (HPC) nodes.

In recent years, the amount of online data processing that the computer performs has increased, thanks especially to the advent of high-performance Graphics Processing Units (GPUs), that, due to their inherently parallel design, are wellsuited for data processing applications. Instruments that combine FPGAs and GPUs are colloquially known as 'heterogeneous' instruments. Such heterogeneous 
back-ends include the Versatile GBT Astronomical Spectrometer (Roshi et al., 2011, also see Chapter 4) and SERENDIP VI (see Chapter 5).

\subsection{SETI}

Organic molecules, the constituents of life on Earth, are long known to be present in the ISM (see Ehrenfreund \& Charnley, 2000, for a review). Organic molecules discovered in the ISM include the sugar glycoaldehyde (Jørgensen et al., 2012) and even the amino acid glycine (Kuan et al., 2003), although the latter detection has been disputed (Snyder et al., 2005; Jones et al., 2007; Cunningham et al., 2007). The formation of relatively complex organic molecules in the interstellar medium points to the idea that such pre-biotic molecules may have been transported to the early Earth by comets and/or meteoroids, seeding life on this planet. By induction, one can come to the conclusion that life may, likewise, have originated on other planets as well.

The emergence of intelligent life, on the other hand, may be more difficult, especially given that it took almost 3.5 billion years since the first living organisms on this planet, for human beings to evolve. Currently, the only feasible way to answer the question of whether other planets also evolved intelligent life that invented technology is to perform a massive, blind, continuous survey of the sky. Due to the technological challenges involved in such an endeavour, so far, only a fraction of the parameter space has been covered, most notably by the project SERENDIP (the Search for Extraterrestrial Radio Emissions from Nearby Developed Intelligent 
Populations; see also Chapter 5).

The number of confirmed extrasolar planets in the Galaxy is $\sim 1000$, while the number of candidate planets is about five times as much ${ }^{6}$. This large (and growing) number of planets provides plenty of opportunities for targeted observations. Siemion et al. (2013a) report targeted observations of 86 potentially habitable extrasolar planets with the GBT, along with a search for narrow-band $(<5 \mathrm{~Hz})$ radio emission. The non-detection of any unambiguously artificial signal yields an upper limit of 1 per cent of transiting planetary systems hosting technological civilizations that transmit within the search parameter space.

\subsubsection{Detection Considerations}

SETI surveys are most commonly done in the radio, infrared, and optical wavelengths. The main focus of most SETI surveys, though, is the radio. Signals that radio SETI mainly focusses on are narrow band emission of spectral line widths not more than a few Hz, and pulsed signals. The expected sources of these emissions include intentional beacons as well as leakage - for example, radio or television broadcasts, or planetary radar (Siemion et al., 2013a).

The aim of most of these surveys is the direct detection of an unambiguously engineered signal. Indirect evidence is also probed, especially in infrared surveys, in the search for 'Dyson spheres' (Dyson, 1960), astro-engineered shells made by hypothetical highly advanced civilizations around a star. These shells would emit secondary thermal radiation in the infrared, and there have been attempts to detect

\footnotetext{
${ }^{6}$ http://planetquest.jpl.nasa.gov
} 
its signature (see, for example, Carrigan, Jr., 2009).

More exotic sources that have been speculated include ETI signals amplified using astrophysical masers (Cordes, 1993), or the artificially modulated emission of radio pulsars (see Chapter 6 ).

\subsubsection{SETI Instrumentation}

SETI instruments are similar to pulsar back-ends in that they are spectrometers. The search is mainly for extremely narrow band signals, with spectral widths on the order of $1 \mathrm{~Hz}$. The rationale behind this is that the narrowest naturally-occurring spectral lines have widths not less than $\sim 550 \mathrm{~Hz}$ (Cohen et al., 1987), so any signal with a bandwidth much less than this is potentially of artificial origin. For this task, unlike pulsar back-ends, SETI spectrometers do not require high time

resolution. Spectra are usually accumulated for about one second to increase the $\mathrm{S} / \mathrm{N}$. Each spectrum is then corrected for its baseline, and a power threshold is applied. Signals above this threshold are stored as potential candidates for later inspection.

Some SETI spectrometers also support searches for pulsed signals. Pulsed signals, like pulsar signals, need to be corrected for dispersion. Data analysis for these kind of signals typically use modified pulsar search pipelines (see, for example, Siemion et al., 2013a). 


\subsection{Dissertation Outline}

The outline of this dissertation is as follows. In Chapter 2, I discuss a Bayesian technique to constraining the luminosity function parameters and population size of radio pulsars in globular clusters. In Chapter 3, I present Bayesian and Monte Carlo approaches to constraining the number of pulsars in the Galactic Center. In Chapter 4, I describe a GPU-based wide-bandwidth spectrometer that forms part of the VEGAS back-end at the GBT. In Chapter 5, I describe the heterogeneous SETI spectrometer SERENDIP VI, to be installed at the GBT. In Chapter 6, I present a novel SETI strategy involving modulation of pulsar signals by ETI. Finally, in

Chapter 7, I present the main conclusions from this work and make suggestions for future progress. 


\section{Chapter 2}

\section{Constraining the luminosity function parameters and population size}

\section{of radio pulsars in globular clusters}

The work in this chapter was originally published as: Jayanth Chennamangalam, D. R. Lorimer, Ilya Mandel and Manjari Bagchi, 'Constraining the luminosity function parameters and population size of radio pulsars in globular clusters', MNRAS, vol. 431, p. 874,2013

\subsection{Introduction}

Globular clusters are spherical collections of stars located throughout the haloes of galaxies. Once thought to be composed entirely of old metal-poor population II stars, they are now also believed to form during interactions or collisions of galaxies, therefore containing younger stars having higher metallicities (see Zepf, 2003). The total masses of globular clusters range up to the order of $10^{6} M_{\odot}$ (see Meylan \& Heggie, 1997), and core stellar number densities reach $10^{6} \mathrm{pc}^{-3}$. The high core densities lead to dynamical interactions between stellar systems that are found less commonly in the Galactic plane. For example, globular clusters favour the formation of low-mass X-ray binaries (LMXBs) that are believed to be the progenitors of millisecond pulsars (MSPs; Alpar et al., 1982; Archibald et al., 2009), and hence, the fraction of MSPs among all pulsars in globular clusters is much larger than that 
in the Galactic field ( $\sim 97$ per cent versus $\sim 11$ per cent). In addition, the binary MSPs in globular clusters tend to have higher eccentricities compared to their field counterparts, due to exchange or fly-by encounters. MSPs, due to their formation history, can be considered long-lived tracers of LMXBs, and therefore, constraints on the MSP content of globular clusters provide unique insights into binary evolution and the integrated dynamical history of globular clusters, while determining the radio luminosity function of these pulsars helps shed light on the radio emission mechanism in action in these compact objects.

Pulsar searches of globular clusters have yielded impressive returns in recent years (see Camilo \& Rasio, 2005), with currently 144 pulsars known in 28 clusters $^{1}$. Of these, three clusters, Terzan 5, 47 Tucanae and M 28 are known to harbour more than 10 pulsars each, the most populous being Terzan 5 with 34 (Ransom S. M., private communication). In this chapter, we describe a Bayesian method that we have developed to compute an estimate of the true number of pulsars in a given cluster, given an observed population. There have been many attempts to constrain the population size of pulsars in all globular clusters in the Galaxy (see, for example, Kulkarni, Narayan \& Romani, 1990; Bagchi, Lorimer \& Chennamangalam, 2011). This work is different in that it treats clusters individually instead of dealing with the total population. Bayesian approaches to constrain the pulsar population of individual globular clusters have been used specifically for the case of young (nonrecycled) pulsars by Boyles et al. (2011). This work focuses on the entire radio

\footnotetext{
${ }^{1}$ See Paulo Freire's globular cluster pulsar catalogue at http://www.naic.edu/ pfreire/ GCpsr.html
} 
pulsar content of the cluster - the majority of which is made up of old (recycled) pulsars - and additionally, attempts to constrain luminosity function parameters jointly with population size.

Faucher-Giguère \& Kaspi (2006) have shown that the luminosity distribution of non-recycled pulsars in the Galactic field appears to be log-normal in form. More recently, Bagchi et al. (2011) have verified that the observed luminosities of recycled pulsars in globular clusters are consistent with this result. Assuming, therefore, that there is no significant difference between the nature of Galactic and cluster populations, we investigate some of the consequences that occur when one applies this functional form to populations of pulsars in individual globular clusters.

For a log-normal (base-10) distribution of pulsar luminosities, the luminosity function is given by

$$
f(\log L)=\frac{1}{\sigma \sqrt{2 \pi}} e^{-\frac{(\log L-\mu)^{2}}{2 \sigma^{2}}},
$$

where $L$ is the luminosity in $\mathrm{mJy} \mathrm{kpc}^{2}, \mu$ is the mean and $\sigma$ is the standard deviation of the distribution. We are interested in the situation where we observe $n$ pulsars with luminosities above some limiting luminosity $L_{\min }$. Given this sample of pulsars, we ask what constraints we can place on their luminosity function parameters, in addition to the potentially observable population size $N$ (that is, the population of pulsars beaming towards the Earth). Another way of thinking about this problem is that there is a family of luminosity function parameters and population sizes that is consistent with an observation of $n$ pulsars above the luminosity limit of the survey, and we are analyzing the posterior probabilities of different members of this family 
given the observations.

This chapter is organized as follows: In $\S 2.2$, we describe our technique. In $\S 2.3$, we apply the technique to observations of a few globular clusters to determine the constraints on the luminosity function parameters and population size. Later, we refine our results using a priori information on the luminosity function parameters to get a better estimate of the number of pulsars in those clusters. A summary and our conclusions are presented in $\S 2.5$.

\subsection{Bayesian parameter estimation}

Bayes' theorem (see Wall \& Jenkins, 2003; Gregory, 2005), for the purpose of parameter estimation, can be stated mathematically as

$$
p(\boldsymbol{\theta} \mid D, M)=\frac{p(D \mid \boldsymbol{\theta}, M) p(\boldsymbol{\theta} \mid M)}{p(D \mid M)},
$$

where $\boldsymbol{\theta}$ is a set of parameters, $D$ is some data and $M$ is a model describing the parameters. In this notation, $p(\boldsymbol{\theta} \mid D, M)$ represents the probability of obtaining a set of parameter values given the data and the model, and is termed the joint posterior probability density. Similarly, $p(D \mid \boldsymbol{\theta}, M)$ is the probability of having obtained the observed data, given the parameter values and the model, and is termed the likelihood, and $p(\boldsymbol{\theta} \mid M)$, the a priori probability dictated by the model, is termed the prior probability density. The denominator, $p(D \mid M)$ is called the evidence, and is

just a normalizing factor that can be dropped since we are only interested in relative 
probabilities, thereby giving

$$
p(\boldsymbol{\theta} \mid D, M) \propto p(D \mid \boldsymbol{\theta}, M) p(\boldsymbol{\theta} \mid M) .
$$

In this chapter, we use Bayes' theorem to find the joint posterior probability density functions of the model parameters $\mu, \sigma$ and $N$ given some data. In our case, the data are the individual pulsar flux densities that we call $\left\{S_{i}\right\}$, the observed number of pulsars, $n$, and the total diffuse flux density of the cluster, $S_{\text {obs }}$.

Luminosity is a property intrinsic to pulsars, while flux density is the corresponding observable. The relationship between the two quantities is given by

$$
L=\frac{4 \pi r^{2}}{\delta} \sin ^{2}\left(\frac{\rho}{2}\right) \int_{\nu_{1}}^{\nu_{2}} S_{\text {mean }}(\nu) d \nu,
$$

where $r$ is the distance to the pulsar, $\delta$ is the pulse duty cycle, $\rho$ is the radius of the pulsar emission cone, $S_{\text {mean }}(\nu)$ is the mean flux density of the pulsar as a function of observing frequency and $\nu_{1}$ and $\nu_{2}$ are the bounds of the frequency range over which the pulsar is observed (see Lorimer \& Kramer, 2005). Due to the uncertainty associated with the beam geometry, the values of $\delta$ and $\rho$ are not generally reliable for luminosity calculations. Therefore, we use a simplified model of the luminosity, the 'pseudo-luminosity', that is defined as $L_{\nu}=S_{\nu} r^{2}$ at a given frequency $\nu$ (the subscript $\nu$ on $L$ and $S$ will be dropped for the rest of the chapter). As can be inferred from Equation (2.4) and the aforementioned pseudo-luminosity equation, the luminosity function is inevitably corrupted by uncertainties in distance. To 
mitigate this, we decided to perform our analysis initially in terms of the measured flux densities, and later, use a model of distance uncertainty to convert our results to the luminosity domain. We take the distance to all pulsars in a globular cluster to be the same. The log-normal in luminosity can then alternatively be written in terms of the flux density. The probability of detecting a pulsar with flux density $S$ in the range $\log S$ to $\log S+d(\log S)$ is then given by a $\log$-normal in $S$ as

$$
p(\log S) d(\log S)=\frac{1}{\sigma_{S} \sqrt{2 \pi}} e^{-\frac{\left(\log S-\mu_{S}\right)^{2}}{2 \sigma_{S}^{2}}} d(\log S),
$$

where $S$ is in mJy, and $\mu_{S}$ and $\sigma_{S}$ are the mean and standard deviation of the flux density distribution. The probability of observing a pulsar above the limit $S_{\min }$ is then

$$
\begin{aligned}
p_{\text {obs }} & =\int_{\log S_{\min }}^{\infty} p(\log S) d(\log S) \\
& =\frac{1}{2} \operatorname{erfc}\left(\frac{\log S_{\min }-\mu_{S}}{\sqrt{2} \sigma_{S}}\right) .
\end{aligned}
$$

Our analysis involves computing three likelihoods in the flux domain based on three sets of data, computing the total likelihood as the product of these three likelihoods, converting this flux domain likelihood to the luminosity domain, and subsequently, applying priors to obtain the posterior. This procedure is depicted graphically in the block diagram of Figure 2.1. 


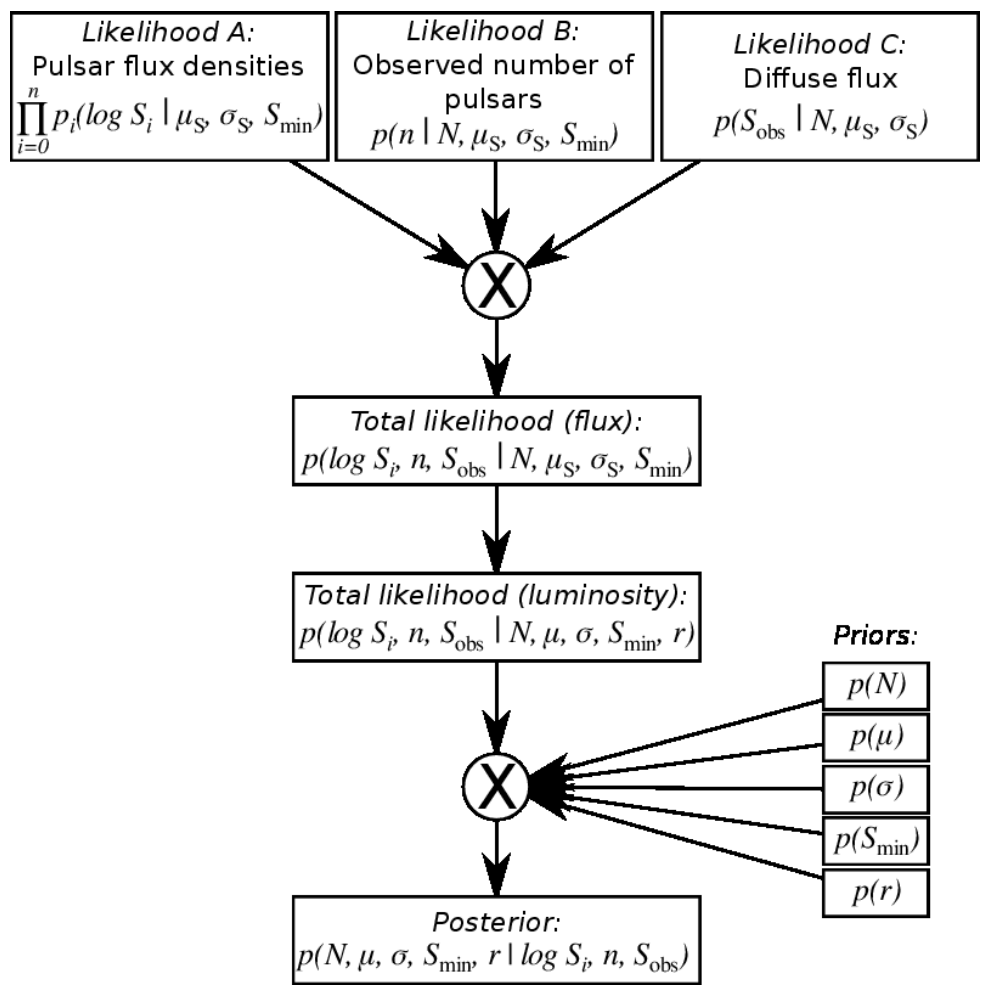

Figure 2.1: Logical flow of the Bayesian analysis. The circle with the $\times$ sign symbolizes a multiplication operation. 


\subsubsection{Using pulsar flux densities}

In the first step, we consider as data the measured flux densities of the pulsars in the cluster under scrutiny, that we call $\left\{S_{i}\right\}$. Ideally, the survey sensitivity limit $S_{\min }$ can be taken as another datum, but its exact value is not always known. The effects of radiometer noise, Doppler smearing, interference, and in some cases, interstellar scintillation, result in a distribution of $S_{\min }$. We decided, therefore, to parametrize $S_{\min }$. The likelihood of observing a set of pulsars with fluxes $\left\{S_{i}\right\}$ is represented as

$$
\prod_{i=1}^{n} p_{i}\left(\log S_{i} \mid \mu_{S}, \sigma_{S}, S_{\min }\right)
$$

where $n$ is the number of observed pulsars in the cluster. Each term in this product is given by

$$
p_{i}\left(\log S_{i} \mid \mu_{S}, \sigma_{S}, S_{\text {min }}\right)=\frac{1}{p_{\text {obs }} \sigma_{S} \sqrt{2 \pi}} e^{-\frac{\left(\log S_{i}-\mu_{S}\right)^{2}}{2 \sigma_{S}^{2}}},
$$

where $p_{\text {obs }}$ is as given in Equation (2.6). This likelihood is represented as 'Likelihood

A' in Figure 2.1. Uncertainties in the flux density measurements are not considered here, but note that ignoring them will have the effect of underestimating the width of posterior credible intervals. In $\S 2.4 .1$, we discuss the effects of ignoring the errors associated with flux density measurements.

\subsubsection{Incorporating the number of observed pulsars}

To infer the total number of pulsars $N$ in the cluster, we follow Boyles et al. (2011) to take as likelihood the probability of observing $n$ pulsars in a cluster with $N$ pulsars, 
given by the binomial distribution

$$
p\left(n \mid N, \mu_{S}, \sigma_{S}, S_{\mathrm{min}}\right)=\frac{N !}{n !(N-n) !} p_{\mathrm{obs}}^{n}\left(1-p_{\mathrm{obs}}\right)^{N-n}
$$

where $p_{\text {obs }}$ is computed as in Equation (2.6). This likelihood is shown as 'Likelihood B' in Figure 2.1.

\subsubsection{Considering diffuse emission}

Next, we incorporate information about the observed diffuse flux $S_{\text {obs }}$ from the direction of the globular cluster. We assume that all radio emission is due to the pulsars in the cluster, including both detected pulsars and the unresolved background. Unlike standard pulsar searches, imaging the diffuse radio emission of a cluster to estimate the number of pulsars therein, is not affected by phenomena that cause pulse broadening, such as dispersion or scattering, or the fact that some of the pulsars in the cluster are in accelerating frames (Fruchter \& Goss, 1990). For the likelihood of measuring the diffuse flux $S_{\text {obs }}$, we choose

$$
p\left(S_{\text {obs }} \mid N, \mu_{S}, \sigma_{S}\right)=\frac{1}{\sigma_{\text {diff }} \sqrt{2 \pi}} e^{-\frac{\left(S_{\text {obs }}-S_{\text {diff }}\right)^{2}}{2 \sigma_{\text {diff }}^{2}}},
$$

where $S_{\text {diff }}$ is the expectation of the total diffuse flux of a cluster whose flux density distribution is a log-normal with parameters $\mu_{S}$ and $\sigma_{S}$, and having $N$ pulsars, and $\sigma_{\text {diff }}$ is the standard deviation of this distribution. This likelihood is referred to as 'Likelihood C' in Figure 2.1. Assuming that the cluster contains $N$ pulsars, each 
having average luminosity,

$$
S_{\text {diff }}=N\langle S\rangle,
$$

and

$$
\sigma_{\text {diff }}=\sqrt{N} \mathrm{SD}(S)
$$

where the expectation of $S$,

$$
\langle S\rangle=10^{\mu_{S}+\frac{1}{2} \sigma_{S}^{2} \ln (10)},
$$

and the standard deviation of $S$,

$$
\mathrm{SD}(S)=10^{\mu_{S}+\frac{1}{2} \sigma_{S}^{2} \ln (10)} \sqrt{10^{\sigma_{S}^{2} \ln (10)}-1} .
$$

We do not consider the uncertainty in the diffuse flux measurement, and as mentioned in $\S 2.2 .1$, this has the effect of underestimating the credible intervals on our posteriors.

The total likelihood, then,

$$
\begin{aligned}
p\left(\log S_{i}, n, S_{\mathrm{obs}} \mid N, \mu_{S}, \sigma_{S}, S_{\mathrm{min}}\right) \\
=\prod_{i=1}^{n} p_{i}\left(\log S_{i} \mid \mu_{S}, \sigma_{S}, S_{\min }\right) \\
\quad \times p\left(n \mid N, \mu_{S}, \sigma_{S}, S_{\min }\right) \\
\quad \times p\left(S_{\mathrm{obs}} \mid N, \mu_{S}, \sigma_{S}\right) .
\end{aligned}
$$




\subsubsection{Transformation to luminosity domain}

The flux density distribution of pulsars in a globular cluster, although proportional to their luminosity distribution, is not suitable for comparing the populations in different clusters, as it depends on the distance to the cluster. It is, therefore, useful to transform the total likelihood obtained in the previous subsection to the luminosity domain. We convert the total likelihood of Equation (2.14) to the luminosity domain in the following way. Since the pseudo-luminosity equation can be written in terms of $\log$ arithms as $\log L=\log S+2 \log r$, where $L$ is in $\mathrm{mJy} \mathrm{kpc}^{2}, S$ is in mJy, and $r$ is in $\mathrm{kpc}$, the means of the two distributions are related additively by the term $2 \log r$, while the standard deviations are the same. Taking into account the uncertainty in distance, we have a distribution of distances, $p(r)$. The total likelihood in the luminosity domain is

$$
\begin{aligned}
p\left(\log S_{i}, n, S_{\text {obs }} \mid N, \mu, \sigma, S_{\min }, r\right) & \\
= & p\left(\log S_{i}, n, S_{\text {obs }} \mid N, \mu_{S}, \sigma_{S}, S_{\text {min }}\right),
\end{aligned}
$$

where $\mu$ and $\mu_{S}$ are related additively as mentioned above, and $\sigma$ and $\sigma_{S}$ are equal. The final joint posterior in luminosity is then given by

$$
\begin{aligned}
& p\left(N, \mu, \sigma, S_{\min }, r \mid \log S_{i}, n, S_{\text {obs }}\right) \\
& \propto \quad p\left(\log S_{i}, n, S_{\text {obs }} \mid N, \mu, \sigma, S_{\min }, r\right) \\
& \quad \times p(N) p(\mu) p(\sigma) p\left(S_{\min }\right) p(r) .
\end{aligned}
$$


The prior on $N$ is taken to be uniform from $n$ to $\infty$, where the upper limit, for the sake of computation, would be a sufficiently large value. We also use uniform priors on the model parameters $\mu$ and $\sigma$. Due to the nature of the uncertainty in determining the exact value of $S_{\min }$, we choose a uniform prior on it in the range $\left(0, \min \left(S_{i}\right)\right]$, where the upper limit is the flux density of the least bright pulsar in the cluster. The prior on $r$ is taken to be a Gaussian. This joint posterior is then integrated over various sets of model parameters to obtain marginalized posteriors.

\subsection{Applications}

We applied our Bayesian technique ${ }^{2}$ to the globular clusters Terzan 5, 47 Tucanae and M 28 (although the clusters we consider contain only recycled known pulsars, the analysis would remain the same even if there were young pulsars in the sample). The choice of clusters was based on the amount of data available. Terzan 5 is the cluster most-suited for this analysis due to the fact that it has a relatively large number of pulsars for which flux density measurements are available. Although Terzan 5 has 34 known pulsars (Ransom S. M., private communication), we take $n=25$, the number of pulsars for which we have flux density measurements. The flux densities of the individual pulsars were collected in a literature survey (Bagchi et al., 2011, and references therein), with the values relevant to this work reproduced in Table 2.1. For Terzan 5, the flux densities we used were scaled from those reported at $1950 \mathrm{MHz}$ by Ransom et al. (2005) and Hessels et al. (2006) to $1400 \mathrm{MHz}$ using

\footnotetext{
${ }^{2}$ The software package that we developed to perform the analysis described in this chapter is available freely for download from http://psrpop.phys.wvu.edu/gcbayes/.
} 
a spectral index, $\alpha=-1.9$ (the mean value for globular cluster MSPs), using the power law $S(\nu) \propto \nu^{\alpha}$. Hessels et al. (2007) and Bagchi et al. (2011) discuss the choice of spectral index in detail. The observed diffuse flux density at $1400 \mathrm{MHz}$ is taken to be $S_{\text {obs }}=5.2 \mathrm{mJy}$ (the sum of the diffuse flux and the fluxes of point sources as reported by Fruchter \& Goss, 2000). The priors used were formed in the following ways. The prior on $N$ was chosen to be a uniform distribution in the range $[n, 500]$, where the upper limit is 150 per cent of the upper limit obtained by Bagchi et al. (2011) (using the values of $\mu$ and $\sigma$ as found by Faucher-Giguère \& Kaspi, 2006) above their upper limit. We note that this prior is sufficiently wide to ensure that the posterior does not rail against the prior boundaries. We chose uniform distributions in the same range of $\mu$ and $\sigma$ as used by Bagchi et al. (2011) as our priors, namely, $[-2.0,0.5]$ and $[0.2,1.4]$, respectively. Survey sensitivity limits were not always available, and additionally, due to a variety of factors mentioned in $\S 2.2 .1$, for all of our analyses, we took $S_{\min }$ to be a uniform distribution in the range $\left(0, \min \left(S_{i}\right)\right]$. The most recent measurement of the distance to Terzan 5 , $r=5.5 \pm 0.9 \mathrm{kpc}$ (Ortolani et al., 2007), was used to model the distance prior. We modelled the distance prior as a Gaussian with mean $5.5 \mathrm{kpc}$ and standard deviation $0.9 \mathrm{kpc}$. Figure 2.2 shows the results of the analysis for Terzan 5 . The mode of the marginalized posterior on $N$, shown in Figure 2.2(b), is 43 and the median with the surrounding 95 per cent credible interval is $142_{-110}^{+310}$. As can be seen from Figures 2.2(b), 2.2(c) and 2.2(d), the constraints we obtain on $N, \mu$ and $\sigma$, respectively, are broad, due to a dearth of flux density measurements. The marginalized posterior on $S_{\min }$, plotted in Figure 2.2(e), shows a strong preference 
for values away from 0 and closer to that of the least bright pulsar observed. The main results are tabulated in Table 2.2 .

For 47 Tucanae and M 28, containing 14 and 9 pulsars each, the individual flux densities used are given in Table 2.1. We took $S_{\text {obs }}=2.0 \mathrm{mJy}(1400 \mathrm{MHz}$ flux as reported by McConnell et al., 2004) for 47 Tucanae, and $S_{\text {obs }}=1.8 \mathrm{mJy}(1400$ MHz flux as reported by Kulkarni et al., 1990) for M 28. The priors on $N$ were taken to be uniform in the intervals $[n, 225]$ for 47 Tucanae and $[n, 400]$ for M 28 , where the upper limits were computed in the same way as we did for Terzan 5. Priors on $S_{\text {min }}$ were formed as in the case of Terzan 5 , in the range $\left(0, \min \left(S_{i}\right)\right]$. The latest distance measurement of $4.69 \pm 0.17 \mathrm{kpc}$ (Woodley et al., 2012) was used to form the distance prior for 47 Tucanae. For M 28, we used $r=5.5 \pm 0.3 \mathrm{kpc}$ (Servillat et al., 2012). The main results for these clusters are tabulated in Table 2.2.

Table 2.1: Flux densities used in the analysis.

\begin{tabular}{lr}
\hline Pulsar & Flux density (mJy) \\
\hline Terzan 5 & $1.91^{3}$ \\
\hline J1748-2446A & $0.68^{3}$ \\
J1748-2446C & $0.08^{3}$ \\
J1748-2446D & $0.09^{3}$ \\
J1748-2446E & $0.07^{3}$ \\
\hline J1748-2446F & \\
\hline
\end{tabular}

\footnotetext{
${ }^{3}$ Based on values reported by Ransom et al. (2005). The fractional uncertainties on these values are $\sim 30$ per cent.
} 
Table 2.1 continued

\begin{tabular}{|c|c|}
\hline Pulsar & Flux density (mJy) \\
\hline J1748-2446G & $0.03^{3}$ \\
\hline $\mathrm{J} 1748-2446 \mathrm{H}$ & $0.03^{3}$ \\
\hline J1748-2446I & $0.05^{3}$ \\
\hline $\mathrm{J} 1748-2446 \mathrm{~J}$ & $0.04^{3}$ \\
\hline J1748-2446K & $0.08^{3}$ \\
\hline $\mathrm{J} 1748-2446 \mathrm{~L}$ & $0.08^{3}$ \\
\hline $\mathrm{J} 1748-2446 \mathrm{M}$ & $0.06^{3}$ \\
\hline $\mathrm{J} 1748-2446 \mathrm{~N}$ & $0.10^{3}$ \\
\hline $\mathrm{J} 1748-2446 \mathrm{O}$ & $0.23^{3}$ \\
\hline $\mathrm{J} 1748-2446 \mathrm{P}$ & $0.14^{3}$ \\
\hline J1748-2446Q & $0.05^{3}$ \\
\hline $\mathrm{J} 1748-2446 \mathrm{R}$ & $0.02^{3}$ \\
\hline $\mathrm{J} 1748-2446 \mathrm{~S}$ & $0.03^{3}$ \\
\hline $\mathrm{J} 1748-2446 \mathrm{~T}$ & $0.04^{3}$ \\
\hline $\mathrm{J} 1748-2446 \mathrm{U}$ & $0.03^{3}$ \\
\hline J1748-2446V & $0.13^{3}$ \\
\hline $\mathrm{J} 1748-2446 \mathrm{~W}$ & $0.04^{3}$ \\
\hline J1748-2446X & $0.03^{3}$ \\
\hline J1748-2446Y & $0.03^{3}$ \\
\hline
\end{tabular}

${ }^{4}$ Based on the value reported by Hessels et al. (2006). The fractional uncertainty on this value is 25 per cent. 
Table 2.1 continued

\begin{tabular}{lr}
\hline Pulsar & Flux density $(\mathrm{mJy})$ \\
\hline J1748-2446ad & $0.15^{4}$ \\
\hline 47 Tucanae & \\
\hline J0023-7204C & $0.36^{5}$ \\
J0024-7204D & $0.22^{5}$ \\
J0024-7205E & $0.21^{5}$ \\
J0024-7204F & $0.15^{5}$ \\
J0024-7204G & $0.05^{5}$ \\
J0024-7204H & $0.09^{5}$ \\
J0024-7204I & $0.09^{5}$ \\
J0023-7203J & $0.54^{5}$ \\
J0024-7204L & $0.04^{5}$ \\
J0023-7205M & $0.07^{5}$ \\
J0024-7204N & $0.03^{5}$ \\
J0024-7204O & $0.10^{5}$ \\
J0024-7204Q & $0.05^{5}$ \\
J0024-7203U & $0.06^{5}$ \\
\hline M 28 & $0.94^{6}$ \\
\hline B1821-24A & $0.07^{6}$ \\
J1824-2452B & \\
\hline & \\
\hline
\end{tabular}

${ }^{5}$ Camilo et al. (2000). The fractional uncertainties on these values range from 10 to 40 per cent.

${ }^{6}$ Bégin (2006). The reported values are 'highly uncertain'. 
Table 2.1 continued

\begin{tabular}{lr}
\hline Pulsar & Flux density $(\mathrm{mJy})$ \\
\hline $\mathrm{J} 1824-2452 \mathrm{C}$ & $0.17^{6}$ \\
$\mathrm{~J} 1824-2452 \mathrm{D}$ & $0.05^{6}$ \\
$\mathrm{~J} 1824-2452 \mathrm{E}$ & $0.06^{6}$ \\
$\mathrm{~J} 1824-2452 \mathrm{~F}$ & $0.08^{6}$ \\
$\mathrm{~J} 1824-2452 \mathrm{G}$ & $0.05^{6}$ \\
$\mathrm{~J} 1824-2452 \mathrm{H}$ & $0.06^{6}$ \\
$\mathrm{~J} 1824-2452 \mathrm{~J}$ & $0.07^{6}$ \\
\hline
\end{tabular}

The value of $N$ can be further refined by considering possible dependences on other physical parameters of globular clusters. In (Turk \& Lorimer, 2013), an empirical Bayesian approach is applied to the set of 95 flux density limits for globular clusters presented in Boyles et al. (2011) in which pulsar abundance as a function of two-body encounter rate, metallicity, cluster mass, etc. is incorporated into the likelihood functions. Note that $N$ is the size of the population of pulsars in the cluster that are beaming towards the Earth. We can include the beaming fraction the fraction of all pulsars beaming towards us - to refine this estimate. Uncertainties notwithstanding, the beaming fraction of millisecond pulsars is generally thought to be greater than 50 per cent (Kramer et al., 1998). This, together with the fact that most pulsars in globular clusters are millisecond pulsars, imply that the true 

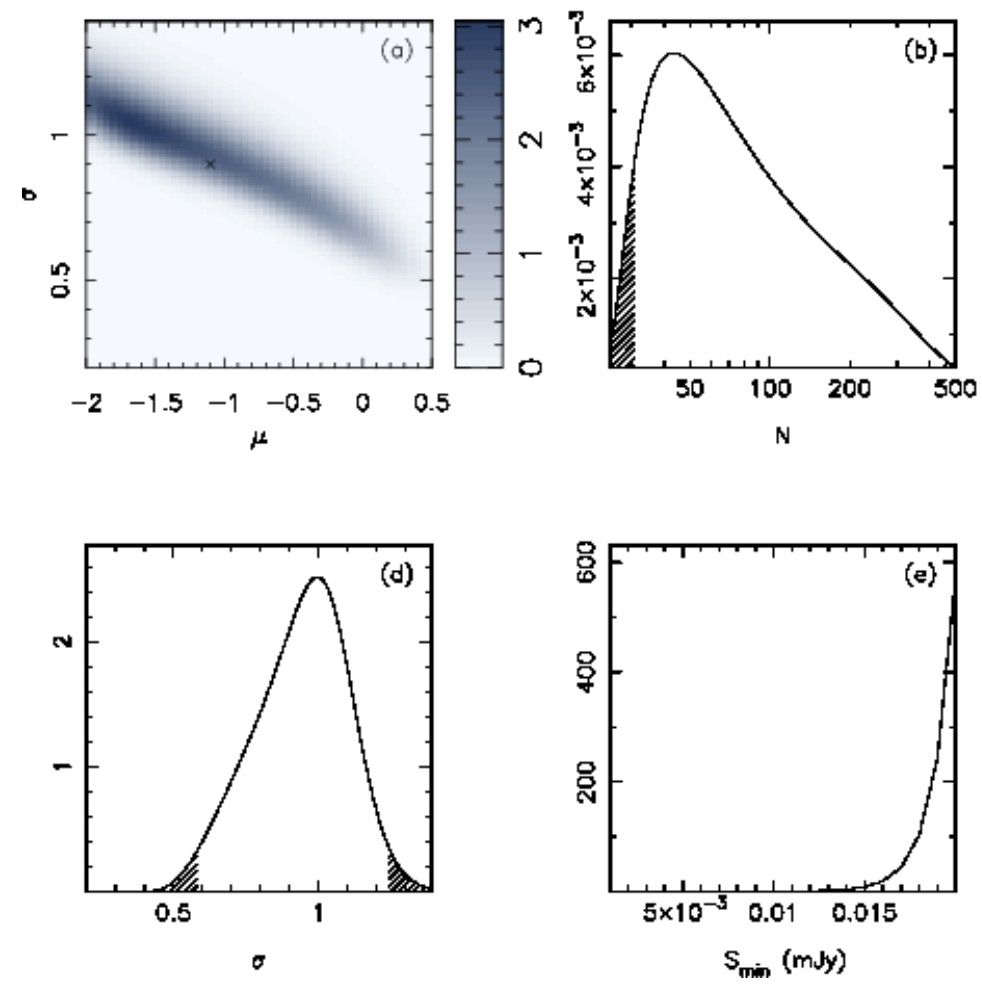

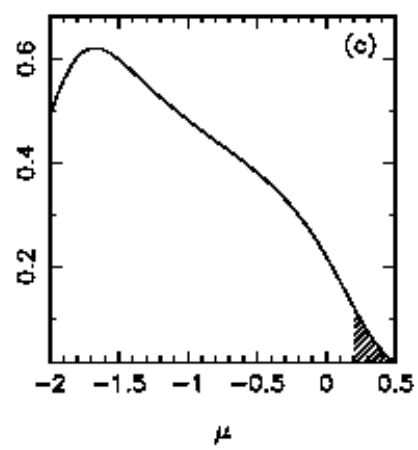

Figure 2.2: Results of the Bayesian analysis for Terzan 5, with $\left\{S_{i}\right\}$ as given in Table 2.1, $n=25$, and $S_{\text {obs }}=5.2 \mathrm{mJy}$. This analysis was run with wide priors on $\mu$ and $\sigma$, with the ranges equal to those used by Bagchi et al. (2011) (their Figure 2). (a) depicts the joint posterior on $\mu$ and $\sigma$, marginalized over $N, S_{\min }$ and $r$. The 'X' symbol marks the intersection of the values obtained by Faucher-Giguère \& Kaspi (2006); (b) is the marginalized posterior for $N$, with a mode of 43 and a median of 142. The $\mathrm{x}$-axis is plotted in log scale for clarity; (c) is the marginalized posterior for $\mu$ with a mode of -1.65 and a median of -1.2 ; (d) is the marginalized posterior for $\sigma$ with a mode of 1.0 and a median of 0.95 ; (e) is the marginalized posterior for $S_{\min }$ with both mode and median equal to $0.02 \mathrm{mJy}$; (f) is the marginalized posterior for $r$, with both mode and median equal to $5.56 \mathrm{kpc}$. The hatching indicates regions that lie outside a 95 per cent credible interval. 
population size in a cluster is approximately a factor of two more than the potentially observable population size.

\subsubsection{Using prior information}

In the framework developed in the previous section, we use broad uniform (noninformative) priors for the mean and standard deviation of the log-normal. This lack of prior information is apparent in Figure 2.2(b), where $N$ is not very well constrained. Prior information can help better constrain the parameters of interest. Boyles et al. (2011) use models of non-recycled Galactic pulsars from Ridley \& Lorimer (2010) to narrow down $\mu$ to between -1.19 and -1.04 , and $\sigma$ to the range 0.91 to 0.98 . We assume that these values are applicable to the globular cluster pulsars based on Bagchi et al. (2011) who draw the conclusion that the luminosity function of cluster pulsars is no different from that of the Galactic disc as found by Faucher-Giguère \& Kaspi (2006). The values themselves are also consistent with the results of Bagchi et al. (2011). We choose $\mu$ and $\sigma$ to be uniform within these ranges. Applying the Bayesian analysis over this narrower range of $\mu$ and $\sigma$ for Terzan 5 results in much tighter constraints on $N$ as seen in Figure 2.3(a), in which the mode of the distribution is 136 and the median and a 95 per cent credible interval is $147_{-65}^{+112}$. The analysis was also performed for 47 Tucanae and M 28, the results of which are given in Figures 2.3(b) and 2.3(c), respectively. For 47 Tucanae, the mode of $N$ is 79 and the median with the surrounding 95 per cent credible interval

is $83_{-35}^{+54}$. For M 28 , the mode is 91 and the median with credible interval is $100_{-52}^{+91}$. 

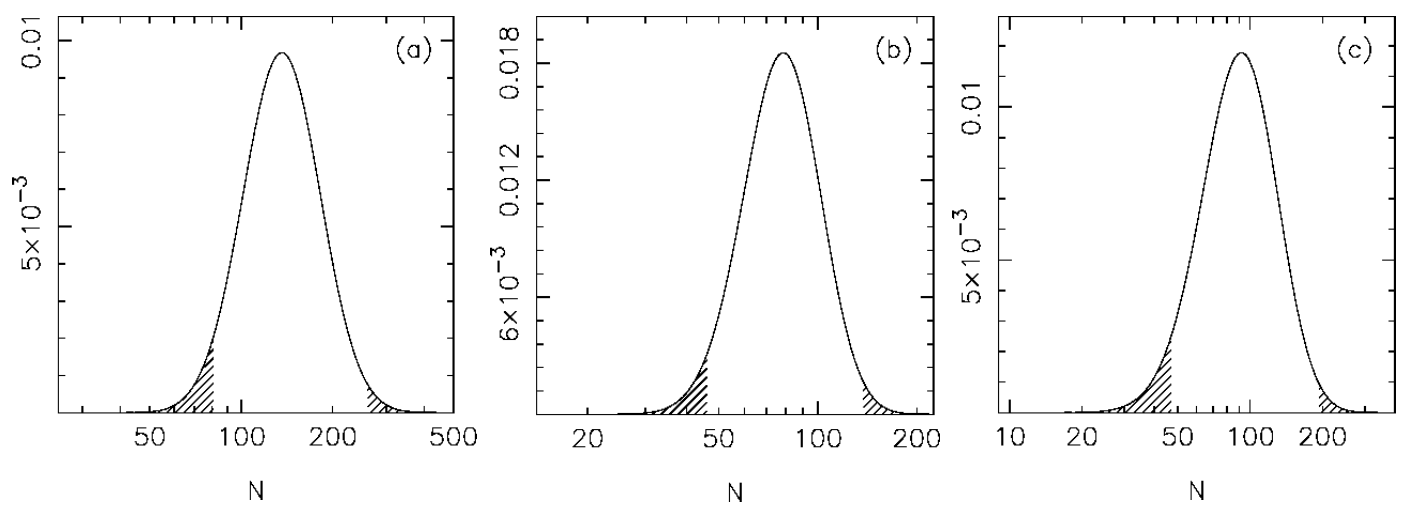

Figure 2.3: Posteriors on $N$ after applying the Boyles et al. (2011) priors on $\mu$ and $\sigma$ : (a) Terzan 5 , with $n=25$ and $S_{\text {obs }}=5.2 \mathrm{mJy}$. The median with the surrounding 95 per cent credible interval of $N$ is $147_{-65}^{+112}$; (b) 47 Tucanae, with $n=14$ and $S_{\text {obs }}=2.0 \mathrm{mJy}$. The median with credible interval is $83_{-35}^{+54}$; (c) M 28 , with $n=9$ and $S_{\text {obs }}=1.8 \mathrm{mJy}$. The median with credible interval is $100_{-52}^{+91}$. The flux densities of the individual pulsars, $\left\{S_{i}\right\}$, used in this analysis are given in Table 2.1.

Our result for Terzan 5 is consistent with that of Bagchi et al. (2011). In the case of 47 Tucanae and M 28, there is partial, yet considerable overlap between our credible ranges and the corresponding confidence intervals of Bagchi et al. (2011). For 47 Tucanae, our results agree with those of Grindlay et al. (2002), i.e., 35-90 MSPs with X-ray luminosities above $10^{30} \mathrm{erg} \mathrm{s}^{-1}$. However, our result for 47 Tucanae is inconsistent with that of McConnell et al. (2004) who estimate $N \leq 30$. This disparity may be due to the high scintillation of the pulsars in this cluster affecting both individual as well as diffuse flux measurements. The results of our analyses are tabulated in Table 2.2. 
Table 2.2: Median and 95 per cent credible intervals from the various analyses presented in this chapter. We note here that, in addition to the sources of error mentioned in the text, the values of $\mu$ and $\sigma$ presented here are also affected by the fact that computations are discrete and hence use a finite number of steps. Note that for the case of narrow priors on $\mu$ and $\sigma$, the corresponding two columns do not carry any useful information, reflecting merely the ranges of the priors, and are included here only for completeness.

\begin{tabular}{lrrr}
\hline Cluster & $N$ & $\mu$ & $\sigma$ \\
\hline Wide priors on $\mu$ and $\sigma$ & & & \\
\hline Ter 5 & $142_{-110}^{+310}$ & $-1.2_{-0.8}^{+1.4}$ & $1.0_{-0.4}^{+0.3}$ \\
47 Tuc & $39_{-25}^{+169}$ & $-0.6_{-1.3}^{+0.9}$ & $0.7_{-0.4}^{+0.4}$ \\
M 28 & $198_{-169}^{+191}$ & $-1.3_{-0.7}^{+1.1}$ & $0.8_{-0.3}^{+0.3}$ \\
\hline Narrow priors on $\mu$ and $\sigma$ & & & \\
\hline Ter 5 & $147_{-65}^{+112}$ & $-1.12_{-0.07}^{+0.08}$ & $0.94_{-0.03}^{+0.03}$ \\
47 Tuc & $83_{-35}^{+54}$ & $-1.13_{-0.07}^{+0.08}$ & $0.94_{-0.03}^{+0.04}$ \\
M 28 & $100_{-52}^{+91}$ & $-1.13_{-0.06}^{+0.09}$ & $0.94_{-0.03}^{+0.04}$ \\
\hline
\end{tabular}

\subsection{Discussion}

\subsubsection{Effect of flux density measurement errors on credible intervals}

Our analysis in its present form does not take into account measurement uncertainties of pulsar flux densities. This leads to an underestimation of our credible intervals and in this section, we discuss the effect that neglecting measurement errors has on our credible intervals. We performed a Monte Carlo simulation in which the flux density corresponding to each Terzan 5 pulsar was modelled as a Gaussian with mean equal to the measured value (given in Table 2.1) and standard deviation equal to the measurement error (given in the footnotes to Table 2.1). A flux density value was picked for each pulsar from these distributions and our Bayesian analysis performed on the new set of flux densities. The analysis was done with both wide and narrow priors on $\mu$ and $\sigma$, resulting in two sets of credible intervals. 
For non-informative priors, the standard deviation on the lower limit of the credible interval for $N$ was found to be 7 while that on the upper limit was 165 . For narrow priors, the standard deviation on the lower limit was 19 while that on the upper limit was 75 . This simplified simulation of the impact of unmodelled measurement uncertainties suggests that the lower limits of the 95 per cent credible intervals are fairly robust, while their upper limits may vary by about one half of the values given in Table 2.2. A more accurate simulation would involve generating sets of flux densities according to all the priors in our analysis, with added dither due to the unmodelled measurement uncertainty, and for each set, compute the 95 per cent credible intervals using our technique, and check what fraction of true values lies outside these intervals. Such a simulation, although more accurate, would be computationally expensive. In principle, measurement uncertainties could be included directly in the likelihood model, and marginalized over to compute the posteriors of interest.

\subsubsection{Effect of increasing detections on credible intervals}

In order to gauge the performance of our technique with respect to increasing pulsar detections and subsequent flux density measurements, we performed the following Monte Carlo simulation. We simulated a globular cluster with population size equal to our wide-prior median estimate for Terzan 5, 142 pulsars, located at the distance of Terzan 5, whose luminosity follows a log-normal with $\mu$ and $\sigma$ fixed at the FaucherGiguère \& Kaspi (2006) values of -1.1 and 0.9 , respectively. For this cluster, we 

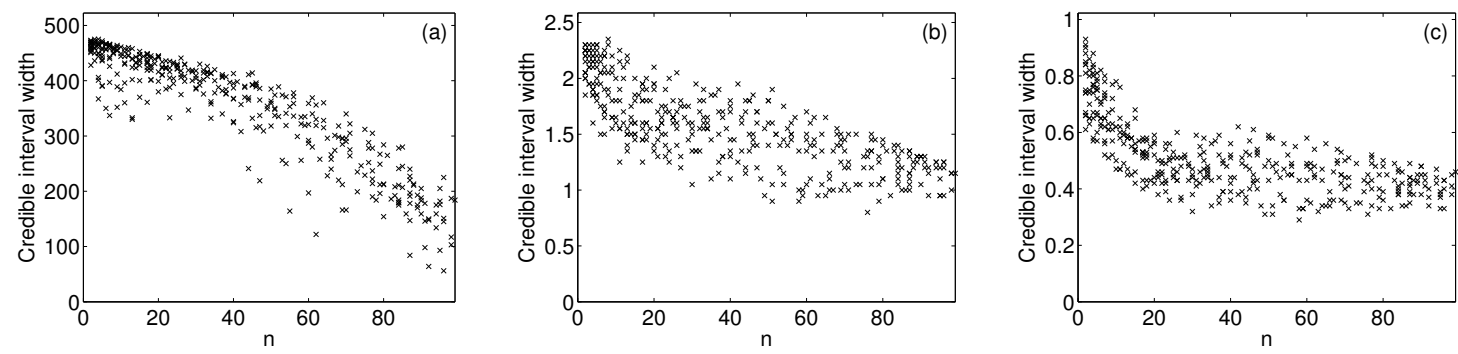

Figure 2.4: Results of the Monte Carlo simulation described in $\S 2.4 .2$. (a) shows how the width of the credible interval on $N$ decreases with increasing number of detected pulsars, $n$. (b) and (c) correspond to credible intervals on $\mu$ and $\sigma$, respectively.

varied the survey sensitivity limit, and at each step, counted the observed number of pulsars, and ran our Bayesian analysis, giving us a set of credible intervals. The Bayesian analysis was done with the same priors as in the first part of $\S 2.3$, viz. uniform prior on $N$ in the range $[n, 500]$, uniform prior on $S_{\min }$ in the range $(0$, $\min \left(S_{i}\right)$ ], and the uniform, wide Bagchi et al. (2011) priors on $\mu$ and $\sigma$. This process was then repeated for multiple Monte Carlo realizations of the log-normal to allow for flux density variations to be manifested. The results are given in Figure 2.4 where the width of the credible intervals on the parameters $N, \mu$ and $\sigma$ are plotted against the number of pulsars detected. As expected, there is a clear improvement in the credible interval widths with the number of pulsars. Since our population estimate of 142 indicates that we have already detected about a fifth of the potentially observable pulsars in Terzan 5, increasing the number of detections/flux density measurements by, say, a factor of 2 would improve our credible interval on $N$ by approximately 15 per cent, whereas the credible intervals on $\mu$ an $\sigma$ would improve by about 15 per cent and 10 per cent, respectively. 


\subsection{Summary and conclusions}

We have developed a Bayesian technique to constrain the luminosity function parameters and population size of pulsars in individual globular clusters, given a data set that consists of the number of observed pulsars, the flux densities of the individual pulsars in the cluster and the total diffuse flux emission from the direction of the globular cluster, assuming a log-normal luminosity function. We have applied our analysis to a few globular clusters and have demonstrated the utility of this technique in constraining the aforementioned parameters.

Our technique is applied in two different ways - first, with no prior information, and second, assuming prior knowledge of the possible ranges of $\mu$ and $\sigma$. As shown for Terzan 5, the results for the first approach do not constrain $N, \mu$ or $\sigma$ very well due to paucity of data, but the latter two do exhibit consistency with the values found by Faucher-Giguère \& Kaspi (2006) and Bagchi et al. (2011). For the second approach in which we assume prior information to bound $\mu$ and $\sigma$, the priors help better constrain the total number of pulsars in the cluster.

The technique we have developed here should prove useful in future studies of the globular cluster luminosity function where ongoing and future pulsar surveys are expected to provide a substantial increase in the observed populations of pulsars in many clusters. In particular, we anticipate that the increased amount of data would enable us to constrain the distributions of $\mu$ and $\sigma$ independently (i.e. without the need to assume prior information from the Galactic pulsar population). Further interferometric measurements of the diffuse radio flux in many globular clusters 
could provide improved constraints on $\mu$ and $\sigma$ by measuring the flux contribution from the individually unresolvable population of pulsars. 


\section{Chapter 3}

\section{The Galactic center pulsar population}

The work in this chapter was originally published as: Jayanth Chennamangalam, D. R. Lorimer, 'The Galactic Centre pulsar population', MNRAS, vol. 440, p. L86, 2014

\subsection{Introduction}

Discovering radio pulsars in the Galactic center (GC) has been a long-sought goal, due to the promise it bears in probing the gravitational field of the massive black hole in the region (e.g., Pfahl \& Loeb, 2004; Liu et al., 2012), and in deciphering the nature of the interstellar medium in its vicinity. Despite several radio surveys (e.g., Kramer et al., 2000; Johnston et al., 2006; Deneva et al., 2009; Deneva, 2010; Macquart et al., 2010; Bates et al., 2011; Eatough et al., 2013a; Siemion et al., 2013b), no pulsars were found. So far, the only pulsar in the inner parsec of the GC was found to be an $\sim 3.76 \mathrm{~s}$ magnetar, which was discovered following an $\mathrm{X}$ ray flaring episode in April 2013 (Kennea et al., 2013), and a subsequent periodicity search (Mori et al., 2013). This was later confirmed in the radio as PSR J1745-2900 (Eatough et al., 2013b; Buttu et al., 2013). Kennea et al. (2013), Mori et al. (2013) and Rea et al. (2013) analysed the X-ray absorption of this source, and found that it is consistent with being at a similar distance as Sgr A*. Rea et al. (2013) localised 
the magnetar to an angular distance of $\sim 2.4^{\prime \prime}$ from Sgr $\mathrm{A}^{*}$. At the GC distance of $8.25 \mathrm{kpc}$ (Genzel et al., 2010), this corresponds to a minimum distance of $\sim 0.1$ pc. Interstellar scattering was thought to be a major problem in detecting pulsars in the GC, as models of electron density dictate a scattering timescale of at least $6.3 \nu_{\mathrm{GHz}}^{-4} \mathrm{~s}$, but potentially up to 200 times larger (Cordes \& Lazio, 1997), indicating that observations at higher frequencies are more favourable, with Macquart et al. (2010) suggesting optimal frequencies in the 10-16 GHz range for searches of nonmillisecond pulsars. However, recent pulse broadening measurements (Spitler et al., 2013) and angular broadening measurements (Bower et al., 2013) of the GC pulsar have demonstrated that scattering may not be as severe a limitation.

The GC magnetar has the largest dispersion measure ever measured for a pulsar, $1778 \pm 3 \mathrm{~cm}^{-3} \mathrm{pc}$, and the largest rotation measure ever measured for any object other than Sgr A* itself, $-66960 \pm 50 \mathrm{rad} \mathrm{m}^{-2}$ (Eatough et al., 2013c; Shannon \& Johnston, 2013). Eatough et al. (2013c) have shown that the large Faraday rotation can be explained by a large magnetic field associated with the plasma within 10 pc of the GC black hole, suggesting a highly complicated and magnetized interstellar medium in the GC region, well suited for scattering electromagnetic radiation. Spitler et al. (2013) measure a pulse broadening timescale of $1.3 \pm 0.2 \mathrm{~s}$ for J1745-2900 at $1 \mathrm{GHz}$, which, albeit large, is much less than predicted values. The lack of detections of previous surveys implies either that previous surveys have not been sensitive enough, or that the GC tends to produce magnetars. Given that observations of the GC have hitherto detected one magnetar and no normal pul- 
sar $^{1}$, in this chapter, we attempt to constrain the number of potentially observable pulsars (including magnetars) in the region using recent studies of the pulsar luminosity function and spectral indices. We employ two complementary methods ${ }^{2}$ - firstly, a Bayesian parameter estimation approach, and secondly, a Monte Carlo (MC) approach to constrain the pulsar population in the GC.

The organization of this chapter is as follows. In $§ 3.2 .1$, we describe our Bayesian technique and apply it to a few past surveys of the GC to obtain upper limits on the the number of GC pulsars. In $\S 3.2 .2$, we describe our MC approach and use it to constrain the number of GC pulsars. In $\S 3.3$, we discuss our results and implications for future surveys.

\subsection{Constraining the GC pulsar content}

\subsubsection{Bayesian Approach}

To quantify the likely size of the pulsar population in the GC region, we treat the GC as a population of pulsars at a common distance from the Earth, $D_{\mathrm{GC}}$, and consider a survey of this region at some frequency $\nu$ as having some finite probability of detecting a pulsar with flux density $S$ and a radio spectral index $\alpha$. Here, as usual, we adopt a power-law relationship for the radio spectra (see, e.g., Lorimer et al., 1995) so that $S \propto \nu^{\alpha}$. For a survey with some limiting sensitivity $S_{\min }$ at frequency

\footnotetext{
${ }^{1}$ In this chapter, we use the term 'normal pulsar' to mean a non-recycled pulsar that is not a magnetar.

${ }^{2}$ The software package that we developed to perform the analysis described in this chapter is available freely for download from http://psrpop.phys.wvu.edu/gcpulsars.
} 
$\nu$, the corresponding limiting pulsar pseudo-luminosity scaled to $1.4 \mathrm{GHz}$

$$
L_{\min }=S_{\min }\left(\frac{1.4 \mathrm{GHz}}{\nu}\right)^{\alpha} D_{\mathrm{GC}}^{2} .
$$

This limiting luminosity can be used to compute the detection probability, i.e., the probability of observing a pulsar above this limit, based on a choice of the pulsar luminosity function. Faucher-Giguère \& Kaspi (2006) have shown that the luminosity distribution of normal pulsars in the Galactic field is log-normal in form. For the log-normal luminosity function, the detection probability

$$
\theta=\frac{1}{2} \operatorname{erfc}\left[\frac{\log L_{\min }-\mu}{\sqrt{2} \sigma}\right],
$$

where $\mu$ and $\sigma$ are the mean and standard deviation of the log-normal.

Using Bayes' theorem, we can utilize the above detection probability to estimate the number of pulsars in the GC. The joint posterior probability density for the number of non-recycled pulsars in the GC and the spectral index, given $n$ observed non-recycled pulsars,

$$
p(N, \alpha \mid n) \propto p(n \mid N, \alpha) p(N) p(\alpha),
$$

where $p(n \mid N, \alpha)$ is the likelihood function, and $p(N)$ and $p(\alpha)$ are the prior probability density functions of $N$ and $\alpha$, respectively. To account for the fact that we have observed one magnetar in the GC and zero normal pulsars, the observed number of non-recycled pulsars is written as $n=n_{\mathrm{np}}+n_{\mathrm{mag}}$, where $n_{\mathrm{np}}=0$ is the 
observed number of normal pulsars and $n_{\text {mag }}=1$ is the observed number of magnetars. The aforementioned Bayesian relation can then be rewritten, and the the likelihood function expanded, as

$$
p\left(N, \alpha \mid n_{\mathrm{np}}, n_{\mathrm{mag}}\right) \propto p\left(n_{\mathrm{np}} \mid N, \alpha\right) p\left(n_{\mathrm{mag}} \mid N, \alpha\right) p(N) p(\alpha) .
$$

Here we make the reasonable assumption of statistical independence for $n_{\mathrm{np}}$ and $n_{\text {mag }}$ given $N$ and $\alpha$, as the formation scenarios for normal pulsars and magnetars are likely different.

The parameter that we are trying to constrain, namely, the total number of non-recycled pulsars $N$, can be written in terms of the magnetar fraction $f$ - the ratio of magnetars to normal pulsars - as

$$
N=N_{\mathrm{np}}+N_{\mathrm{mag}}=N(1-f)+N f
$$

The value of $f$ is highly uncertain. In the Galactic field, 25 magnetars are known ${ }^{3}$, at least four of which emit in the radio, and of which only one was found in a radio survey (Levin et al., 2010). There are about 2000 non-recycled pulsars in the field $^{4}$ (Manchester et al., 2005), giving $f \approx 0.01$. Considering only radio-emitting magnetars, $f$ becomes 0.002 , and considering only radio-loud magnetars detected in surveys, the magnetar fraction reduces to 0.0005. Due to the intermittency in the radio emission of magnetars, together with the selection effects that plague radio

\footnotetext{
${ }^{3} \mathrm{http}: / /$ www .physics.mcgill.ca/ pulsar/magnetar/main.html

${ }^{4}$ http://www.atnf.csiro.au/people/pulsar/psrcat/
} 
surveys, the exact value of the magnetar fraction is unknown. So we decided to parametrize $f$ in our analysis, giving

$$
\begin{aligned}
p\left(N, f, \alpha \mid n_{\mathrm{np}}, n_{\mathrm{mag}}\right) \propto & p\left(n_{\mathrm{np}} \mid N, f, \alpha\right) p\left(n_{\mathrm{mag}} \mid N, f, \alpha\right) \\
& \times p(N) p(f) p(\alpha) .
\end{aligned}
$$

We compute the two likelihood functions using the binomial probability distribution, following Boyles et al. (2011). In the case of normal pulsars, we have

$$
p\left(n_{\mathrm{np}} \mid N, f, \alpha\right)=(1-\theta)^{N(1-f)},
$$

and for magnetars,

$$
p\left(n_{\mathrm{mag}} \mid N, f, \alpha\right)=N f \gamma(1-\gamma)^{N f-1},
$$

where $\gamma$ is the magnetar detection probability. Magnetars are characterized by a flat spectral index, i.e., their luminosities appear to be independent of the observing frequency. Under this assumption we derive the magnetar detection probability from the normal pulsar detection probability as

$$
\gamma=\theta(\alpha=0)=\frac{1}{2} \operatorname{erfc}\left[\frac{\log \left(S_{\min } D_{\mathrm{GC}}^{2}\right)-\mu}{\sqrt{2} \sigma}\right],
$$

and $p\left(n_{\mathrm{mag}} \mid N, f, \alpha\right)$ becomes $p\left(n_{\mathrm{mag}} \mid N, f\right)$.

To avoid any bias in our analysis, we adopt non-informative priors for $N$ and $f$ (i.e., uniform probability within given ranges stated below). For $\alpha$, we use the 
results of Bates et al. ( 2013) and take

$$
p(\alpha) \propto e^{-\frac{(\alpha-\bar{\alpha})^{2}}{2 \sigma_{\alpha}^{2}}},
$$

where $\bar{\alpha}=-1.41$ is the mean spectral index and $\sigma_{\alpha}=0.96$ is the standard deviation. The final Bayesian relation can then be written as

$$
\begin{aligned}
p\left(N, f, \alpha \mid n_{\mathrm{np}}, n_{\mathrm{mag}}\right) \propto & (1-\theta)^{N(1-f)} N f \gamma(1-\gamma)^{N f-1} \\
& \times p(N) p(f) e^{-\frac{(\alpha-\bar{\alpha})^{2}}{2 \sigma_{\alpha}^{2}}} .
\end{aligned}
$$

This is then integrated over $f$ and $\alpha$ to obtain the marginalized posterior of $N$, $p\left(N \mid n_{\mathrm{np}}, n_{\mathrm{mag}}\right)$.

We applied our technique to the surveys of the GC analyzed by Wharton et al. (2012), namely those discussed in Johnston et al. (2006), Deneva (2010), Macquart et al. (2010) and Bates et al. (2011). In applying our analysis to these past surveys, we made the reasonable assumption that, had the magnetar become active earlier, all these surveys would have detected it. We computed the survey sensitivity limits based on information provided in those papers, and additionally performed a normalization to ensure that the minimum flux density values are average values over the part of the beam that cover the inner 1 pc of the GC, modelling each beam as a Gaussian. We used the broad ranges of $\left[1,10^{5}\right]$ for $N$ and $[0.001,0.999]$ for $f$. We found that for the more sensitive surveys (Deneva, 2010; Macquart et al., 2010), the mean of the posterior on $N$ is in the range 800-3000 and the 99 per cent upper limit is in the range 12000-47000. Our results are tabulated in Table 3.1. 


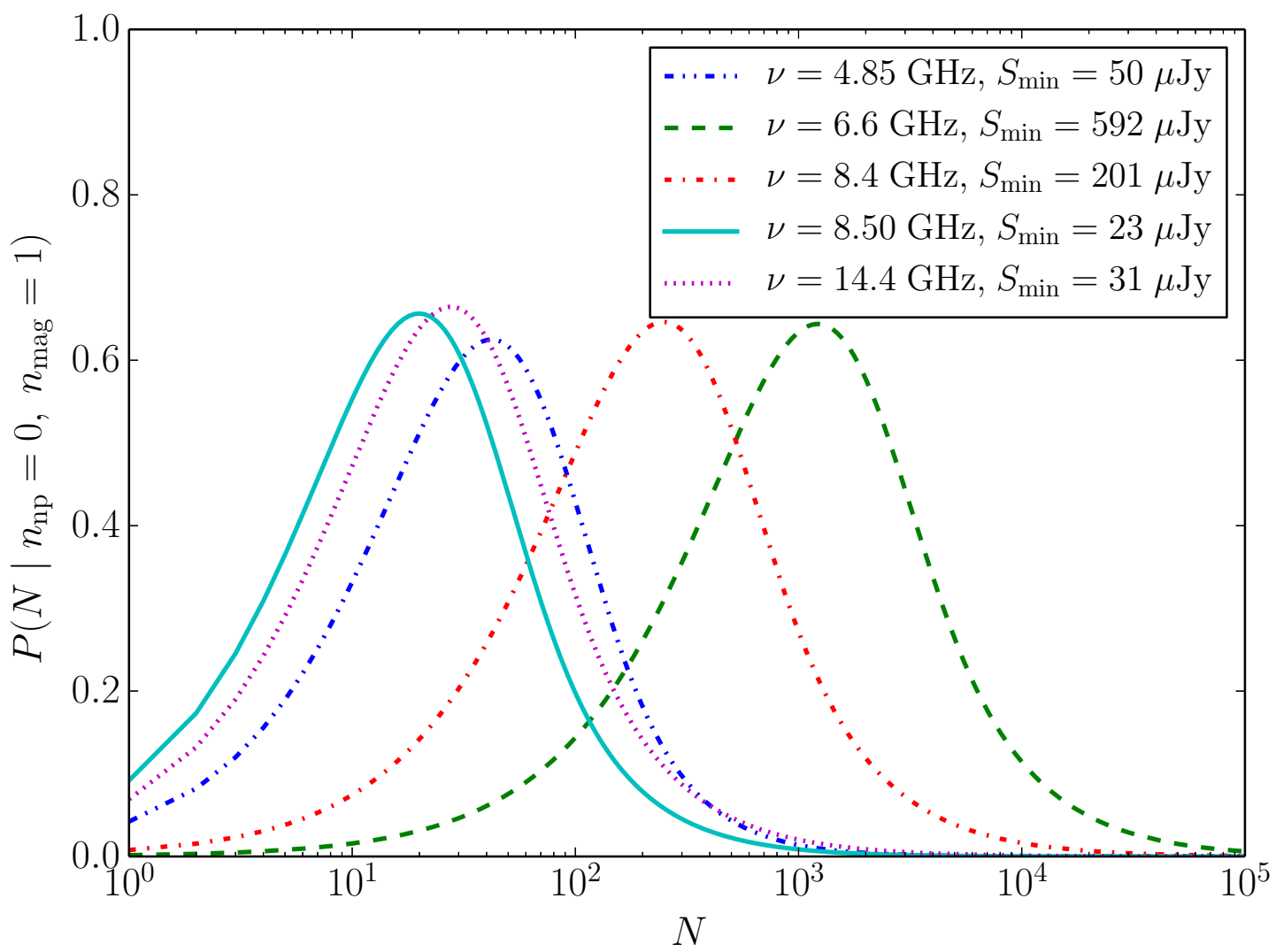

Figure 3.1: Posterior probability density functions of $N$ for each of the surveys listed in Table 3.1.

Figure 3.1 shows the posterior probability density functions of $N$ derived from each of the surveys listed in Table 3.1.

As mentioned previously, the magnetar fraction in the Galactic field is uncertain, and that in the GC is unknown. To study how the 99 per cent upper limit on $N$ would vary with magnetar fraction, instead of using a wide prior on $f$, we chose delta functions in the range $(0.0,1.0)$, and for each of those magnetar fractions, we computed the upper limit. Figure 3.2 shows the results of this analysis. For a small magnetar fraction, the upper limits would be close to the values reported in Table 3.1. If, on the other hand, formation of radio-loud magnetars are somehow 
Table 3.1: Results of our analysis for a few GC surveys. $\langle N\rangle$ is the expected value of $N$ while $N_{\max }$ is the 99 per cent upper limit on $N$.

\begin{tabular}{ccrll}
\hline $\begin{array}{l}\nu \\
(\mathrm{GHz})\end{array}$ & $\begin{array}{c}S_{\min } \\
(\mu \mathrm{Jy})\end{array}$ & $\langle N\rangle$ & $N_{\max }$ & Survey reference \\
\hline 4.85 & 50 & 800 & 12000 & Deneva $(2010)$ \\
6.6 & 592 & 16000 & 92000 & Bates et al. (2011) \\
8.4 & 201 & 9000 & 86000 & Johnston et al. (2006) \\
8.50 & 23 & 1200 & 21000 & Deneva (2010) \\
14.4 & 31 & 3000 & 47000 & Macquart et al. $(2010)$ \\
\hline
\end{tabular}

favoured in the GC region, the fact that we have detected one magnetar implies that we can expect a smaller population size.

\subsubsection{Monte Carlo Approach}

The Bayesian technique described above is relatively agnostic about the period and luminosity evolution of normal pulsars. To make use of the results known from studies of normal pulsars in the Galaxy (see, e.g., Faucher-Giguère \& Kaspi, 2006), we perform an MC simulation of the GC pulsar population and apply it to the Deneva (2010) survey. In this method, we follow Faucher-Giguère \& Kaspi (2006) to simulate a population of $N_{\text {sim }}$ pulsars, evolved over time starting from the distributions of birth spin period, surface magnetic field at birth and age. Picking $N_{\text {sim }}$ from the range $\left[5 \times 10^{3}, 8 \times 10^{4}\right]$, we compute the number of pulsars that have not crossed the death line, i.e., the number of radio-loud pulsars, denoted by $N_{\mathrm{GC}}$. We then apply radiation beaming correction and compute the number of potentially observable pulsars. Given that Spitler et al. (2013) measure the scattering timescale at $1 \mathrm{GHz}$ as $\sim 1.3 \mathrm{~s}$, we scale it to the observation frequency with a power-law 


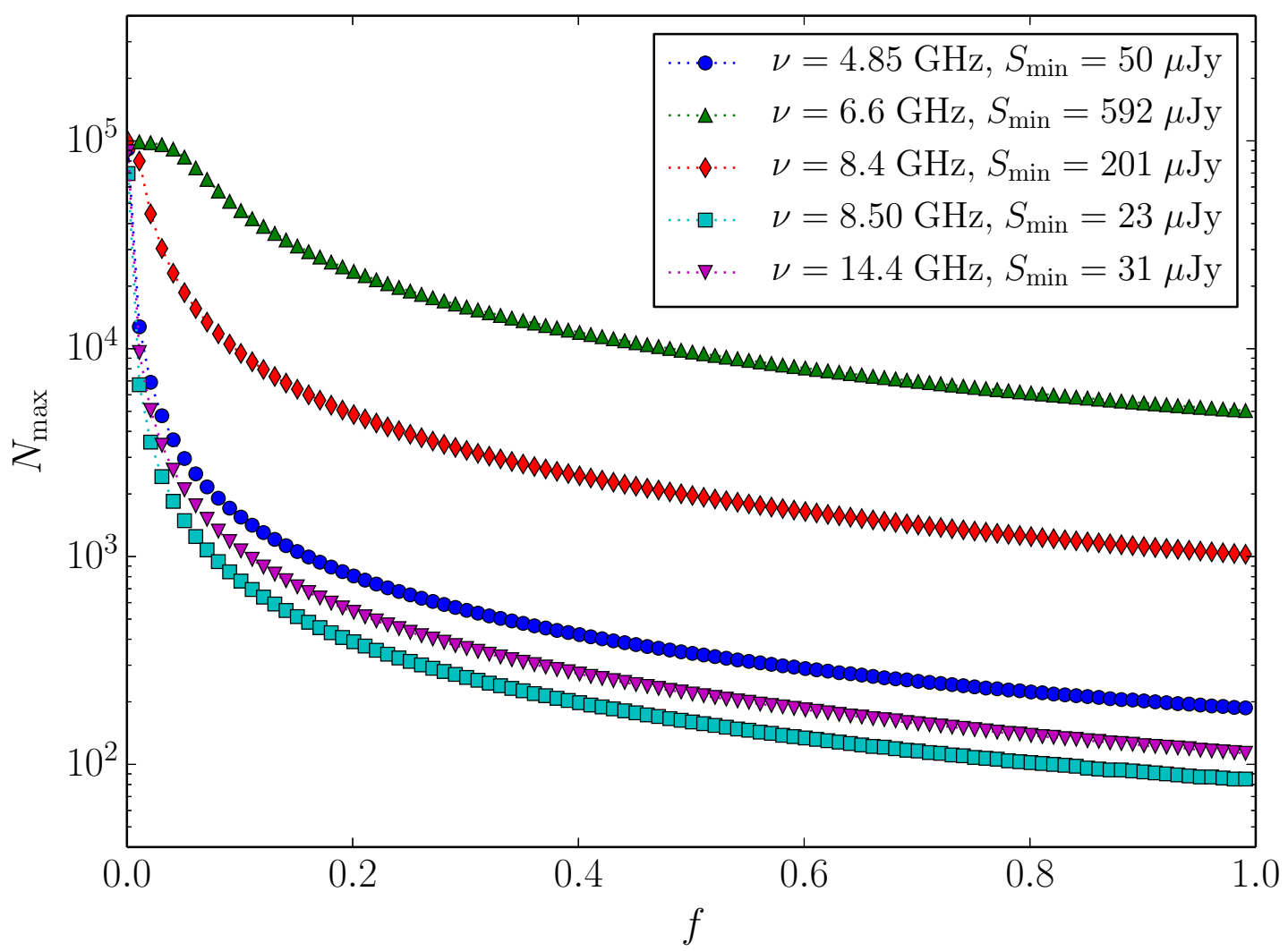

Figure 3.2: The 99 per cent upper limit on $N, N_{\max }$, as a function of magnetar fraction, $f$, for each of the surveys listed in Table 3.1. 
spectral index $\alpha_{\tau}=-3.8$ to compute the scatter-broadened pulse widths for each pulsar. We then compute the luminosities $L$ at $1.4 \mathrm{GHz}$, followed by computations of the signal-to-noise ratio $(S / N)$. We then apply the $S / N$ threshold of the survey to get the detectable number of pulsars, denoted by $n_{\mathrm{obs}}$.

For each value of $N_{\text {sim }}, 10^{4} \mathrm{MC}$ realizations were generated to ensure stability in the mean of $n_{\text {obs }}$. Figure 3.3 shows a plot of observed number of pulsars along with both 68.3 and 99.7 per cent confidence limits versus the mean value of the number of potentially observable pulsars in the $\mathrm{GC},\langle N\rangle$, and the mean value of the total number of radio-emitting pulsars in the region, $\left\langle N_{\mathrm{GC}}\right\rangle$. As can be seen, the lower limits of the 99.7 per cent confidence intervals become inconsistent with an actual detection of zero normal pulsars for $\langle N\rangle$ around 150. Any number $\langle N\rangle \gtrsim 150$ would mean that more pulsars should have been observed. The fact that none have been detected gives an upper bound to the number of potentially observable pulsars in the GC. The assumptions that go into this simulation are that the luminosity function of GC pulsars is the same as that of field pulsars, and that the age distribution of pulsars in the GC follow the uniform distribution for field pulsars (i.e., a constant formation rate). Although a burst of supernovae has been proposed to have occurred in the GC 10 Myr ago (Sofue, 1994), near-infrared observations have revealed some evidence that the star formation rate in the region has been roughly constant over the past $\sim 10$ Gyr (Figer et al., 2004). If these assumptions, including those about the birth spin period and magnetic field distributions are applicable to magnetars as well, the upper limit on the potentially observable population size (of both normal pulsars and magnetars) increases to approximately 200. 


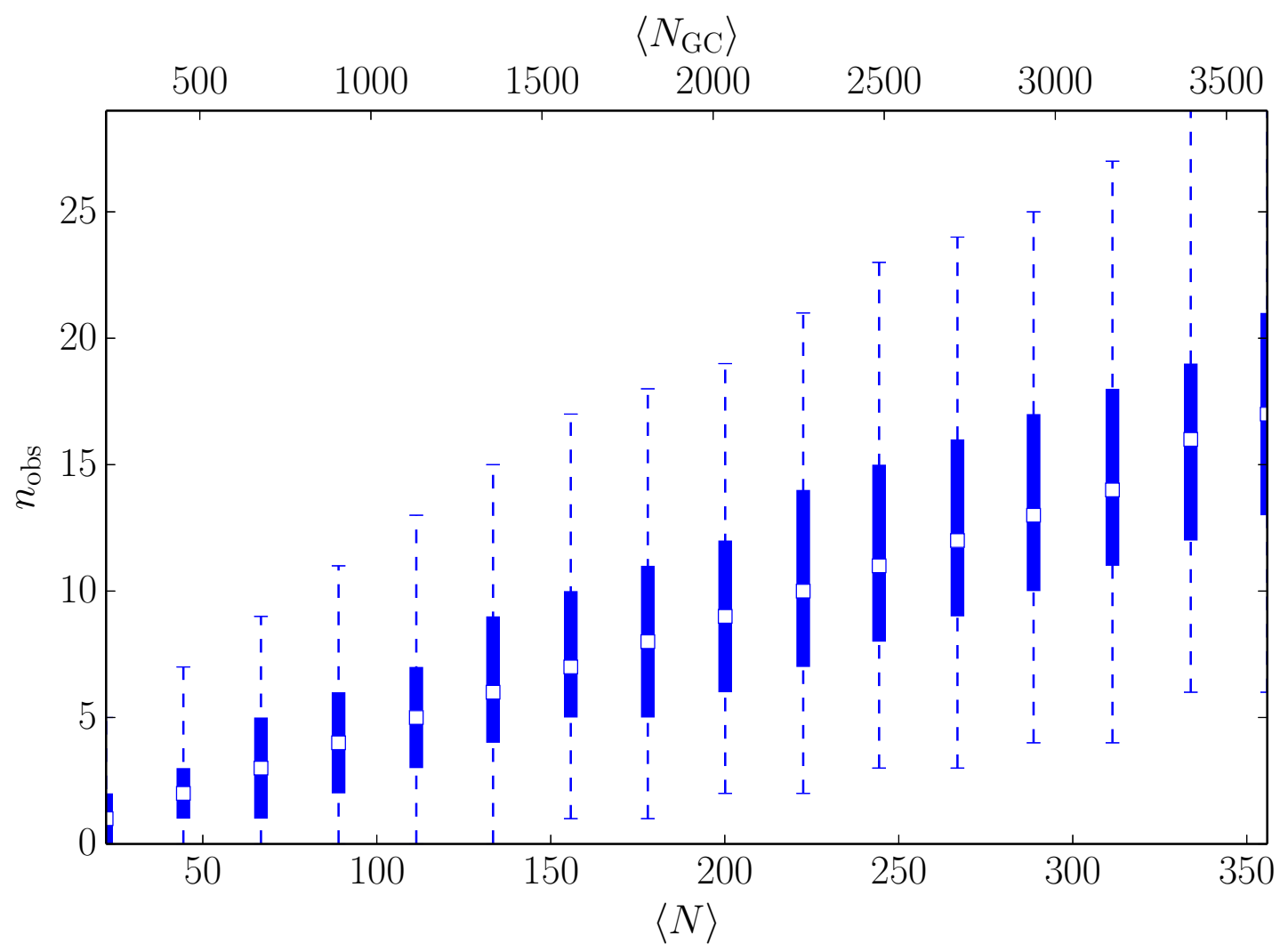

Figure 3.3: The number of observed pulsars, $n_{\mathrm{obs}}$ versus the mean of the number of potentially observable pulsars, $\langle N\rangle$ and the mean of the total number of radioemitting pulsars in the $\mathrm{GC},\left\langle N_{\mathrm{GC}}\right\rangle$. Each point represents $10^{4} \mathrm{MC}$ realizations. The white markers indicate the mean of $n_{\text {obs }}$ and the thick error bars represent the corresponding 68.3 per cent confidence intervals. The dashed error bars are 99.7 per cent confidence limits. 


\subsection{Discussion}

The analyses we have performed in this chapter differ from that of Wharton et al. (2012) mainly in that we use a more realistic luminosity function. Whereas Wharton et al. (2012) used a power-law luminosity function, it is more appropriate to use the log-normal as found by Faucher-Giguère \& Kaspi (2006). We also take into account the recent discovery of one magnetar in the GC, and, in the case of the Bayesian analysis, the fraction of magnetar to normal pulsars in the field. An important assumption we make here is that scattering in the inner parsec is uniform and is consistent with that of the line of sight to the GC magnetar. This appears to be a reasonable assumption, given that Bower et al. (2013) have shown that the angular sizes of the magnetar and Sgr $A^{*}$ are consistent with both sources being behind the same scattering screen. The results of our Bayesian analysis suggest that the population of potentially observable pulsars in the inner parsec of the GC could be as large as several thousand, whereas our MC analysis yields an upper limit of $\sim 200$. The reason the Bayesian analysis yields a broader constraint is because it makes fewer assumptions than the MC method. While the former only assumes a form for the luminosity distribution, the latter makes assumptions about the spindown behaviour and formation rate of pulsars in the GC. We note that, for a typical radio pulsar beaming fraction of $\sim 10$ per cent (Tauris \& Manchester, 1998), the total number of radio-emitting pulsars in the region, for either method, would be an order of magnitude larger. As per the $\mathrm{MC}$ method, the value of $\left\langle n_{\mathrm{obs}}\right\rangle$ corresponding to an actual detection of one pulsar is $\sim 7$. This is consistent with the results of 
Dexter \& O'Leary (2013) who use an estimate of the number of massive stars in the GC, a model of natal kick velocity, and the observed interstellar scattering to get $\left\langle n_{\text {obs }}\right\rangle \approx 10$. Our conservative upper limit of $\sim 200$ suggests that there may not be any detectable pulsar close enough to Sgr A* to probe the gravitational field of the GC black hole. However, Kocsis et al. ( 2012) have suggested that pulsars in the inner parsec that are not close enough to $\mathrm{Sgr} \mathrm{A}^{*}$ can still be useful in detecting intermediate and stellar mass black holes in orbit around the GC black hole.

How many pulsars can we expect to see in future surveys of the GC? To answer this question, we started with the most constraining posterior on $N$ obtained using the Bayesian method, based on the Deneva (2010) survey. We performed MC simulations similar to those described in $§ 3.2 .2$, with the following differences: (i) Instead of picking a set of equi-spaced values, we picked $N$ randomly from the Bayesian posterior; (ii) We did not apply any beaming correction, as the Bayesian posterior gives the number of potentially observable pulsars. The rest of the simulation proceeds as described before, yielding an observed number of pulsars $n_{\mathrm{obs}}$. We performed these MC simulations for a few hypothetical surveys using the Green Bank Telescope (GBT), for each of its receivers from L-band to K-band. The backend used was assumed to be able to sample the maximum instantaneous bandwidths supplied by the receivers. For the GBT K-band Focal Plane Array receiver, we assumed that it was configured to use the VEGAS ${ }^{5}$ backend such that the instantaneous bandwidth sampled is $8750 \mathrm{MHz}$. The sensitivity of each survey was calculated based on a minimum signal-to-noise ratio of 8 , a duty cycle of 10 per cent and an observation time

\footnotetext{
${ }^{5}$ http://www.gb.nrao.edu/vegas
} 
of 7 hours, which is approximately the duration for which the GC is visible from Green Bank. The receiver temperature, receiver gain, number of polarizations and bandwidth used in our sensitivity calculations were taken from the GBT Proposer's Guide $^{6}$. The GC background temperatures were calculated from peak flux densities and spectral indices reported in Law et al. (2008). For each survey, we repeated the simulation $10^{4}$ times to ensure that the mean value stabilizes, and computed the mean and standard deviation for the number of detections. Our results are tabulated in Table 3.2. Encouragingly, our results suggest that there are some prospects for a detection with surveys of this sensitivity in the near future. We caution, however, that in spite of the large number of pulsars we estimate to be present in the GC, and the fact that these surveys have high sensitivity, we may yet detect no pulsar.

As a self-consistency test, to verify that the Bayesian technique we developed in $§ 3.2 .1$ and used to predict $n_{\mathrm{obs}}$ as described above actually works, we applied the above MC simulations to all the past surveys listed in Table 3.1, again using our most constraining posterior on $N$. We also applied it to the most sensitive survey of the GC at $1.4 \mathrm{GHz}$, the Parkes Multi-beam Pulsar Survey (PMPS; Manchester et al., 2001; Morris et al., 2002). The results of these MC simulations are tabulated in Table 3.2. For all past surveys, we obtained 1-sigma limits of the number of detected pulsars that are consistent with zero.

Our MC technique described in $§ 3.2 .2$ yields a conservative upper limit of $~$ 200 potentially observable pulsars, whereas our Bayesian technique yields broader constraints that are an order of magnitude larger. Further deep surveys of the GC

\footnotetext{
${ }^{6}$ https://science.nrao.edu/facilities/gbt/proposing/GBTpg.pdf
} 
Table 3.2: Predictions for surveys, both past and future, based on our most constraining posterior on $N$. Here, $n_{\text {obs }}$ is the mean value of the number of detectable pulsars, given along with 68.3 per cent confidence limits. Values have been rounded to the nearest integer, and the lower limits have been truncated at 0 .

\begin{tabular}{lcrrl}
\hline Survey & $\begin{array}{l}\nu \\
(\mathrm{GHz})\end{array}$ & $\begin{array}{l}S_{\min } \\
(\mu \mathrm{Jy})\end{array}$ & $\begin{array}{r}T_{\mathrm{GC}} \\
(\mathrm{K})\end{array}$ & $n_{\text {obs }}$ \\
\hline Past surveys & & & & \\
\hline PMPS & 1.374 & 3519 & 690 & $0 \pm 0$ \\
Deneva (2010) & 4.85 & 50 & 285 & $1_{-1}^{+0}$ \\
Bates et al. (2011) & 6.6 & 592 & 90 & $0 \pm 0$ \\
Johnston et al. (2006) & 8.4 & 201 & 90 & $0 \pm 0$ \\
Deneva (2010) & 8.50 & 23 & 116 & $3_{-3}^{+0}$ \\
Macquart et al. (2010) & 14.4 & 31 & 103 & $2_{-2}^{+0}$ \\
\hline Future GBT surveys & & & & \\
\hline L-Band & 1.45 & 105 & 435 & $0 \pm 0$ \\
S-Band & 2.165 & 75 & 373 & $1_{-1}^{+0}$ \\
C-Band & 5.0 & 41 & 285 & $2_{-2}^{+0}$ \\
X-Band & 9.2 & 17 & 116 & $3_{-3}^{+0}$ \\
Ku-Band & 13.7 & 14 & 103 & $4_{-4}^{+0}$ \\
K-Band & 22.375 & 10 & 83 & $6_{-6}^{+0}$ \\
\hline
\end{tabular}


in the radio, and monitoring for X-ray outbursts from potential magnetars in the region will help conclusively establish the size of the GC pulsar population. 


\section{Chapter 4}

\section{A GPU-Based Wide-Band Radio Spectrometer}

\subsection{Introduction}

Astronomical data acquisition and online reduction of data are steadily becoming increasingly resource-intensive, not just for new and upcoming telescopes such as the Low Frequency Array (LOFAR) and the Square Kilometre Array (SKA), but also for new instruments at established facilities. Field Programmable Gate Arrays (FPGAs) have long been used at the output of Analog-to-Digital Converters (ADCs) for data reduction and/or packetization, followed by a computer that manages the recording of data to disk. Due to the large amount of data acquired to meet cuttingedge science goals, the need for high-performance and flexible computing resources for data reduction has increased. FPGAs have traditionally been considered suitable for high-bandwidth applications, but the relative difficulty in programming them and the lack of support for floating-point arithmetic, coupled with the relatively inexpensive pricing of Graphics Processing Unit (GPU) cards, have made the advent of GPUs in astronomical instrumentation inevitable. Several real-time GPU-based signal processing systems intended for pulsar astronomy have been developed in recent years (Ransom et al., 2009; Magro et al., 2011; Armour et al., 2012; Barsdell et al., 2012; Magro et al., 2013). Many new instruments being built combine the

high-bandwidth data acquisition capability of FPGAs with the high-performance 
data reduction capability of GPUs, glueing them together with high-throughput networking hardware. Such a heterogeneous architecture is expected to scale up to meet the data-handling requirements of future instruments and telescopes.

In this chapter, we give an overview of a heterogeneous, wide-bandwidth, multi-beam spectrometer that we have built for the Green Bank Telescope (GBT), and describe in detail the GPU-based spectrometry code and its performance. This spectrometer forms part of 'Versatile GBT Astronomical Spectrometer' (VEGAS) (Roshi et al., 2011), an instrument being built by the Collaboration for Astronomy Signal Processing and Electronics Research (CASPER). VEGAS has multiple modes of operation that are broadly classified into two categories - the so-called high bandwidth (HBW) and low-bandwidth (LBW) modes. The HBW mode spectrometry takes place exclusively on Field-Programmable Gate Array (FPGA) boards ${ }^{1}$, with spectra sent to data recording PCs. The LBW modes, on the other hand, involve heterogeneous instrumentation, combining FPGAs for data acquisition followed by GPUs for spectrometry. These modes are based on GPUs as they require a larger number of channels (up to 524,288) than what FPGAs can support. Although these are 'low-bandwidth' modes in the context of VEGAS, these modes are based on code that can support a per-polarization bandwidth of up to $800 \mathrm{MHz}$.

The organization of this chapter is as follows. In $§ 4.2$, we describe the GPUprogramming paradigm, in $\S 4.3$ we describe our software, and in $\S 4.5$ we explain our benchmarking procedure and performance figures.

\footnotetext{
${ }^{1}$ The Reconfigurable Open Architecture Computing Hardware II (ROACH II) platform.
} 


\subsection{The GPU-Programming Paradigm}

In the past, computing performance was improved most commonly by using smaller silicon features and increasing the clock rate. Since computer designers are no longer able to increase the clock rate further due to power constraints, parallelization is the primary method to improve performance in recent times. The Central Processing Unit (CPU) of a typical personal computer (PC) has traditionally contained a single instruction-processing core that can perform only one operation at a time. Multi-tasking on a PC powered by such a CPU is usually achieved by interleaving tasks in time. This obviously degrades the performance of time-critical tasks such as rendering graphics for computer games. One solution to this problem is to offload graphics processing to a dedicated co-processor, the GPU. The GPU contains multiple processing cores that enables it to run multiple instructions simultaneously $^{2}$. This parallelization makes it suitable not just for graphics processing, but also for general purpose computing that requires high performance. Modern GPUs are designed with this in mind, and programming platforms are available that let developers take advantage of this computing power.

The most common General Purpose GPU (GP-GPU) programming platform is Compute Unified Device Architecture (CUDA). CUDA lets developers access the hardware (the parallel compute engine in the GPU) using programming instructions (CUDA Instruction Set Architecture). This is enabled by extending the C language to invoke routines that run on the GPU and using CUDA libraries for numerical

\footnotetext{
${ }^{2}$ Even though modern CPUs contain multiple processing cores (on the order of tens of cores), modern GPUs far surpass them, having cores on the order of hundreds to thousands.
} 


\begin{tabular}{|c|c|c|c|c|}
\hline $\begin{array}{l}\text { Number } \\
\text { of sub- } \\
\text { bands } \\
\text { per pol. }\end{array}$ & $\begin{array}{l}\text { Sub- } \\
\text { band } \\
\text { band- } \\
\text { width } \\
\text { (MHz) }\end{array}$ & $\begin{array}{l}\text { Number } \\
\text { of chan- } \\
\text { nels per } \\
\text { sub- } \\
\text { band } \\
\text { per pol. }\end{array}$ & $\begin{array}{l}\text { Spectral } \\
\text { resolu- } \\
\text { tion } \\
\quad(\mathrm{KHz})\end{array}$ & $\begin{array}{l}\text { Min. } \\
\text { inte- } \\
\text { gration } \\
\text { time } \\
\quad(\mathrm{ms})\end{array}$ \\
\hline 1 & $100.0-187.5$ & $2768-131072$ & $0.8-5.7$ & $10-30$ \\
\hline 1 & $11.72-23.44$ & $32768-524288$ & $0.02-0.7$ & $5-75$ \\
\hline 8 & $15.625-23.44$ & $4096-65536$ & $0.24-5.7$ & $5-100$ \\
\hline
\end{tabular}

Table 4.1: Heterogeneous modes of operation of VEGAS and their specifications. computation and signal processing, such as CUDA Basic Linear Algebra Subroutines (CUBLAS) and CUDA Fast Fourier Transform (CUFFT).

Since GPUs are suitable computing platforms for data-parallel applications, they are increasingly used as dedicated co-processors for data analysis applications that use the high-performance hardware to accelerate their time-critical paths. This also makes GPUs ideal for data-acquisition instruments such as VEGAS.

\subsection{Overview of VEGAS}

The heterogeneous modes of operation of VEGAS ${ }^{3}$ and their specifications are given in Table 4.1. These modes are divided into single-sub-band modes and 8-sub-band modes. The single-sub-band modes can have 32768 to 524288 channels, with subband bandwidths in the range $11.72 \mathrm{MHz}$ to $187.5 \mathrm{MHz}$, whereas the 8-sub-band modes have 4096 to 32768 channels with bandwidths ranging from $15.625 \mathrm{MHz}$ to 23.44 MHz.

Figure 4.1 shows a block diagram of the VEGAS heterogeneous-mode data

\footnotetext{
${ }^{3}$ www.gb.nrao.edu/vegas
} 


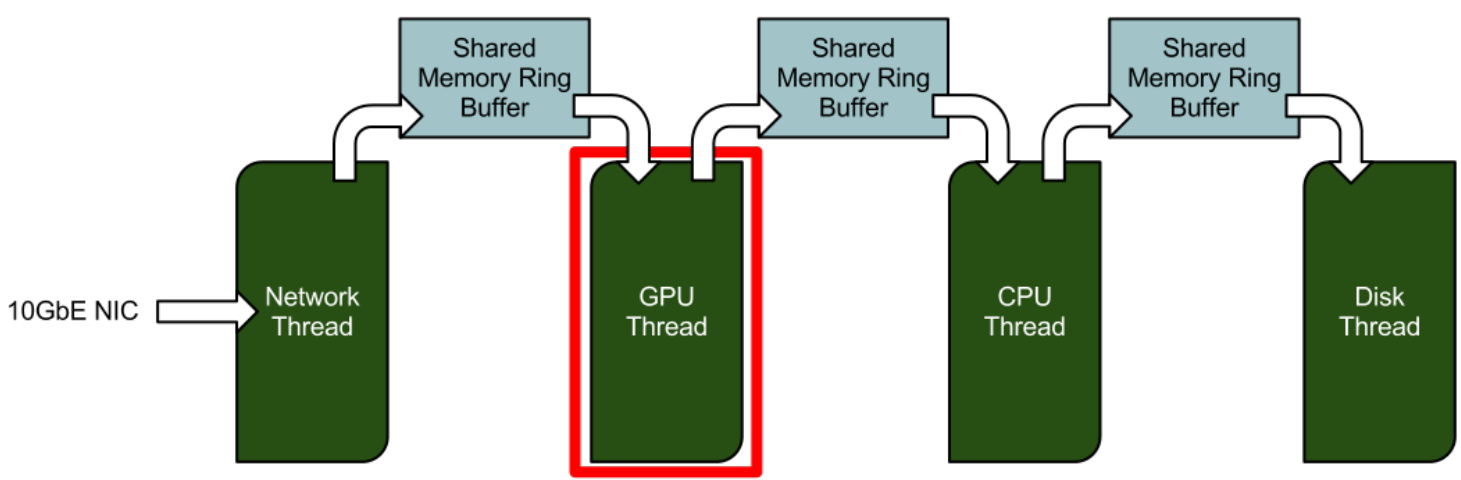

Figure 4.1: Data flow diagram of the software part of the VEGAS data-acquisition pipeline. This paper focuses on the software used in the GPU thread.

pipeline. In the heterogeneous modes, the FPGA board packetizes the signal sampled by an ADC and sends it over 10-Gigabit Ethernet (10GbE) to a PC with a GPU. The VEGAS software pipeline, based on the Green-Bank Ultimate Pulsar Processing Instrument (GUPPI) (Ransom et al., 2009) is made up of multiple concurrent threads, each associated with a separate CPU core. The first thread, called the 'network thread' reads packets off the network and writes the payload to a shared memory ring buffer. The next thread, called the 'GPU thread' reads the data off the buffer and performs spectrometry. Once the accumulated spectra are ready, the output is written to another ring buffer from which the third thread the 'CPU thread' - reads data and performs further accumulation as needed. Once this is done, the output is sent to the 'disk thread' that writes it to disk. Whereas this chapter describes the spectrometer implemented in the GPU thread, a more detailed description of the entire VEGAS software pipeline will appear in Ford et al. (in preparation). 


\subsection{The GPU Spectrometer}

The Discrete Fourier Transform (DFT) of a time series $x_{t}$ is defined as

$$
X_{f}=\sum_{t=0}^{N-1} x_{t} e^{-i t 2 \pi f / N}
$$

where $X_{f}$ is the amplitude of the $f$-th bin in the frequency domain and $N$ is the length of the transform (see, for example, Bracewell, 1999). Since the DFT operates on a finite length of time samples, the frequency domain response of the DFT output bin is a sinc function, with side lobes spreading across the entire bandwidth. This 'spectral leakage', and the related phenomenon of 'scalloping loss' - due to the nonflat nature of the main lobe of the sinc function - can be mitigated by suppressing the side-lobes of the sinc function and changing the single-bin frequency response of the DFT to approximate a rectangular function. This is achieved by a pre-filter introduced before the DFT in which a time series of length $N \times P=M$ to be transformed is weighted by a sinc window function, the length of data is split into $P$

subsets of length $N$ each, and summed point-by-point. The weighting and summing together form essentially a filtering operation in which the elements of the window function are the filter coefficients. The output of the pre-filter is

$$
\begin{aligned}
y_{m} & =\sum_{n=0}^{N-1} \sum_{p=0}^{P-1} x(m N+p N+n) h(p N+n) \\
& \equiv \sum_{n=0}^{N-1} \sum_{p=0}^{P-1} x(m N+p N+n) g_{n}(p),
\end{aligned}
$$




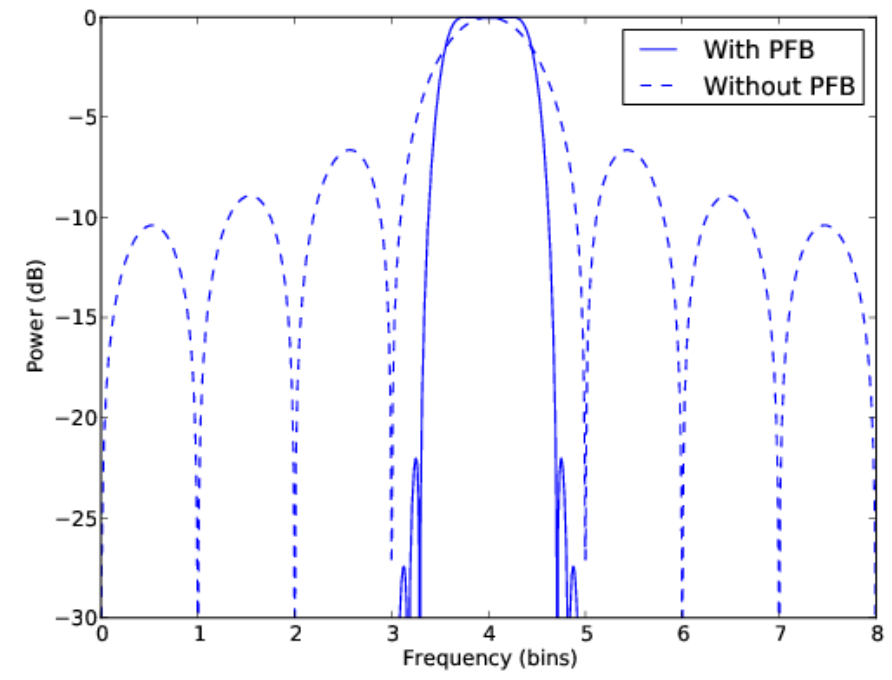

Figure 4.2: Single-bin frequency response of an 8-tap PFB (solid) and a direct FFT (dashed).

where the coefficients $g_{n}(p) \equiv h(p N+n)$ correspond to what are called $P$-tap 'polyphase sub-filters'. The $N$ such polyphase sub-filters that make up this operation, together with the Fourier transform stage, are collectively called a 'polyphase filter bank' ('PFB'). In practice, the weight function is a sinc multiplied by another smoothing function, which, in our case, is a Hanning window. Figure 4.2 shows a comparison between the single-bin responses of a PFB and that of a direct DFT.

The GPU spectrometer described in this chapter implements an 8-tap polyphase filter bank, composed of a pre-filter followed by a Fast Fourier Transform (FFT) stage. 


\subsubsection{The Spectrometer Algorithm}

The input data to our PFB spectrometer is made up of dual-polarization, 8-bit, complex-valued samples, while the output contains $X^{2}, Y^{2}, \Re\left(X Y^{*}\right)$, and $\Im\left(X Y^{*}\right)$, where $X$ is the magnitude spectrum of the horizontal polarization and $Y$ is the magnitude spectrum of the vertical polarization. Note that full-Stokes spectra can easily be generated from these values.

The high-level algorithm of the spectrometer is as follows. Here, following GPU-programming parlance, 'host' indicates code and memory that is associated with the CPU, whereas 'device' indicates code and memory that is associated with the GPU.

1. Initialization

(a) Load filter coefficients

(b) Create FFT plan

2. Copy time series data to device

3. Perform pre-filtering

\section{Perform FFT}

5. Accumulate spectra for desired duration

6. Copy spectra to host

This process is repeated until the program is terminated. A flow chart of the algorithm is given in Figure 4.3. 


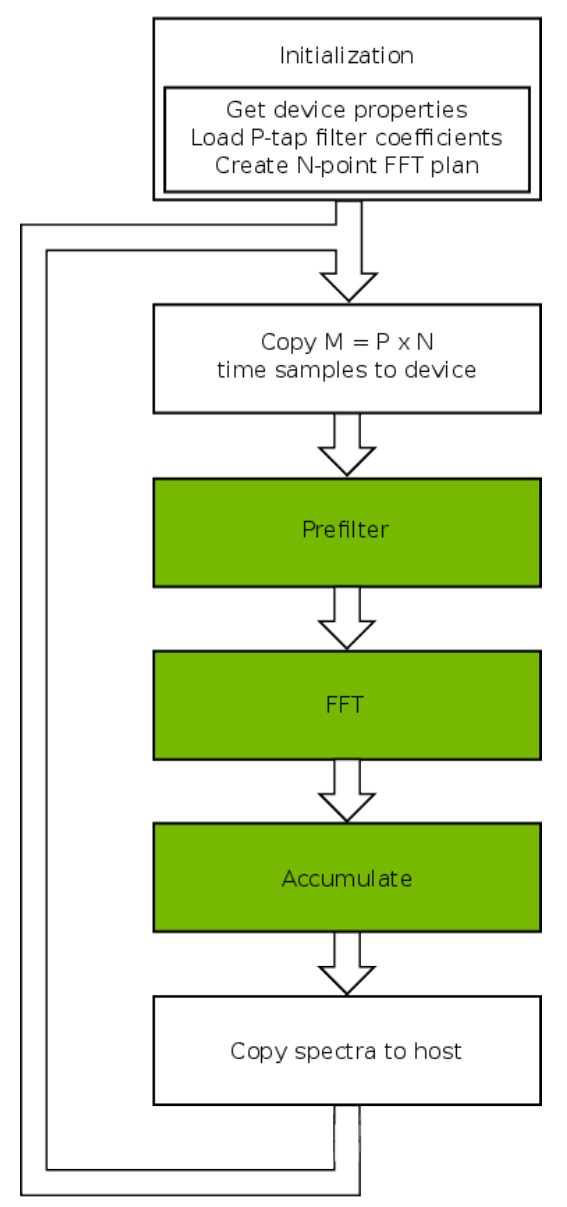

Figure 4.3: Flowchart of the spectrometry process. The green blocks depict operations that run on the GPU. 


\subsection{Benchmarking and Performance Results}

Benchmarking of the software spectrometer was performed on a server-class PC running a flavor of the Linux operating system, with an NVIDIA GeForce GTX 580 commercial (gaming) GPU card. A stand-alone version of the spectrometer program was used, wherein data was read off disk files and pre-loaded in memory, to simulate reading from the shared memory ring buffers of VEGAS described in $\S 4.3$. The test suite was run a large number of times and we report the average values. The peak bandwidth achieved was $\sim 638 \mathrm{MHz}$ (dual-polarization), corresponding to a data rate of $\sim 10.2$ Gbps which is more than what a $10 \mathrm{GbE}$ link can support. The peak performance was achieved for an FFT length of $2^{16}$, with long integrations (accumulation length of 1000). When PFB was turned off, the peak bandwidth achieved was $\sim 794 \mathrm{MHz}$, corresponding to a data rate of $\sim 12.7$ Gbps, again more than what is supported by $10 \mathrm{GbE}$. This peak was for $2^{20}$-point FFT with an accumulation length of 1000. For the VEGAS settings of Table 4.1 (single-sub-band $2^{15}$-point FFT, or 8-sub-band $2^{12}$-point FFT, with an accumulation length of 58 corresponding to a time of $10 \mathrm{~ms}$, and PFB turned on), we achieved a bandwidth of $\sim 537 \mathrm{MHz}$ corresponding to a data rate of $\sim 8.3 \mathrm{Gbps}$. Within the VEGAS framework, with modifications that enabled the code to read the shared memory buffer, we achieved a bandwidth of $\sim 500 \mathrm{MHz}$ (corresponding to $\sim 8$ Gbps). The performance of the code as a function of transform length and accumulation length is depicted in Figure 4.4 . 

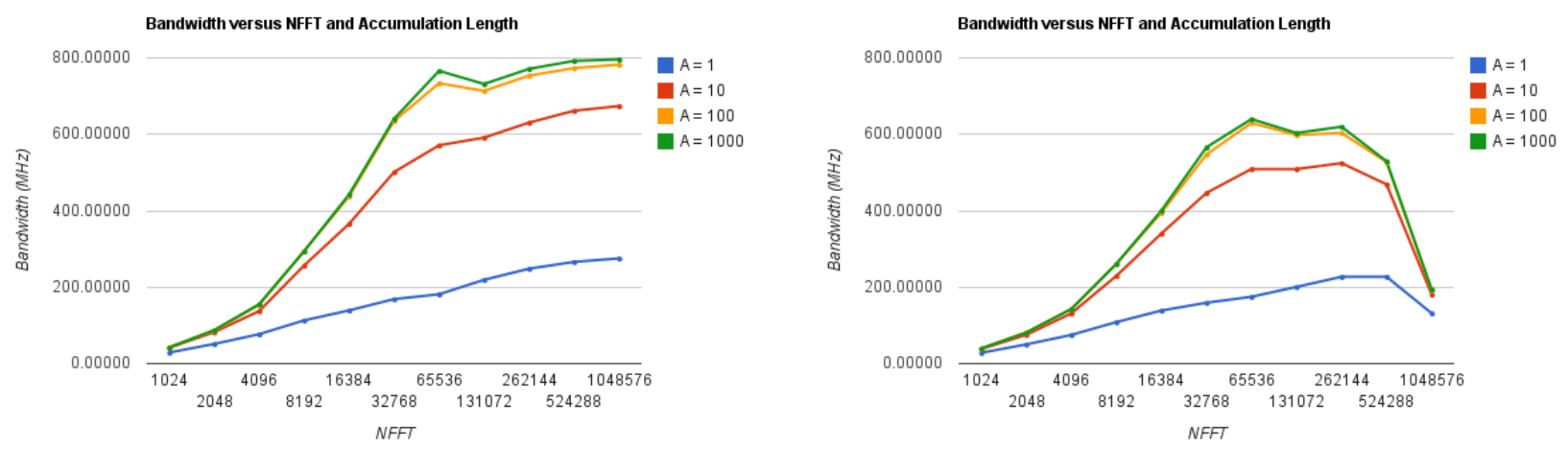

Figure 4.4: Performance of the spectrometer (a) without PFB and (b) with PFB.

\subsection{Conclusions}

We have developed a GPU-based PFB-enabled spectrometer that supports a dualpolarization bandwidth of up to $600 \mathrm{MHz}$ (or a single-polarization bandwidth of up to $1.2 \mathrm{GHz}$ ). Without PFB, it supports a dual-polarization bandwidth of up to $800 \mathrm{MHz}$ (or a single-polarization bandwidth of up to $1.6 \mathrm{GHz}$ ). This bandwidth is sufficient for most spectral-line observations and for some pulsar observations. Future work would involve improving the performance of this software. The simplest way to speed it up would be to implement support for scalability, for example, to enable the software to take advantage of the newer dual-GPU cards, and to support multiple GPU cards on the same PC. Additionally, algorithm-level and further codelevel optimizations may also have the potential to yield higher performance. 


\section{Chapter 5}

\section{SERENDIP VI}

\subsection{Introduction}

The Search for Extraterrestrial Intelligence (SETI) is an on-going effort to detect signals from beyond the Solar System, originating in engineered processes outside the realm of human activity, that are indicative of the presence of extraterrestrial intelligent life. Such efforts cover mainly radio wavelengths, indicative of 'leakage' of communication or radar transmission, or 'beacons' whose purpose is to announce the presence of intelligent life to prospective listeners (see, for example, Siemion et al., 2013a); optical wavelengths, for signals produced by powerful lasers (see, for example, Howard et al., 2007); and infrared wavelengths, the proposed secondary thermal emission from engineered structures such as Dyson spheres (Dyson, 1960; Carrigan, Jr., 2009, for instance). The searches conducted so far have covered only a tiny portion of the parameter space, and as expected, have yielded no detections, but new and upcoming telescopes with all-sky monitoring capabilities, such as LOFAR, MWA, and SKA, hold the promise of increasing the odds of detection.

One of the on-going projects in radio SETI is the Search for Extraterrestrial Radio Emissions from Nearby Developed Intelligent Populations (SERENDIP) at the Arecibo observatory, now in its 34th year (see Bowyer, 2011). The primary aim of SERENDIP is to find narrow-band radio emissions from extraterrestrial intel- 
ligence (ETI). In this work, we present the latest installment in a series of spectrometers built as part of the project. SERENDIP VI is a spectrometer that makes use of hardware developed by the Collaboration for Astronomy Signal Processing and Electronics Research ${ }^{1}$ (CASPER), and commodity computing elements. It is a heterogeneous instrument, that is, it utilizes both Field-Programmable Gate Arrays (FPGAs) and Graphics Processing Units (GPUs) for signal processing and computation. SERENDIP VI is intended for commensal observations at both Arecibo and Green Bank observatories.

The Arecibo version of the spectrometer is a 7-beam back-end, with $300 \mathrm{MHz}$ of bandwidth per beam, per polarization, while the Green Bank Telescope (GBT) machine is a single-pixel back-end, with a bandwidth of $2.5 \mathrm{GHz}$ per polarization. In this chapter, we describe the version of the instrument that will be installed at the GBT. The organization of this chapter is as follows. In $\S 5.2$, we describe the system architecture, in $\S 5.3$, we describe the FPGA design, in $\S 5.4$, we describe the data transport framework, and in $\S 5.5$, we describe the GPU spectrometry algorithm, before presenting preliminary results and development status and concluding in $\S 5.6$.

\subsection{System Architecture}

SERENDIP VI is a high-resolution digital spectrometer, with a spectral resolution of $\sim 1 \mathrm{~Hz}$. The GBT version of the instrument features a bandwidth of $2.5 \mathrm{GHz}$ per polarization, for a single beam. Following the CASPER principle of achieving

\footnotetext{
${ }^{1}$ http://casper.berkeley.edu/
} 
modular architecture by interconnecting computing nodes using an Ethernet fabric, SERENDIP VI comprises of FPGA boards and server-class computers with commercial off-the-shelf gaming GPUs interconnected using a 10 Gigabit Ethernet (10GbE) switch. Figure 5.1 shows the architecture of system. A single FPGA board is used, namely, the Reconfigurable Open Architecture Computing Hardware (ROACH) II, developed by CASPER. The ROACH II board is equipped with two 5 Gsps Analog to Digital Converter (ADC) boards that digitize the IF signals, one per polarization channel. The board also has mezzanine cards equipped with SFP + Ethernet ports that support a data rate of up to 10 Gbps per port. The Ethernet switch is a 48-port device with SFP + ports, and interconnects the ROACH II board with the cluster of High Performance Computing (HPC) nodes. The HPC nodes are server-class PCs with dual hex-core Intel Xeon processors with Sandy Bridge architecture, clocked at $2.3 \mathrm{GHz}$, with $32 \mathrm{~GB}$ RAM. Each HPC node has two Mellanox 10GbE Network Interface Cards (NICs) and two GPUs, such that each node can theoretically process data at a rate of up to 20 Gbps. The GPUs used are NVIDIA GeForce GTX 780 Ti, with 3 GB RAM. The HPC nodes also come with SATA hard disk drives for recording data. SERENDIP VI uses a total of four HPC nodes.

\subsection{FPGA Design}

The FPGA design, developed by the National Radio Astronomy Observatory (NRAO), digitizes data, packs it in UDP frames and transmits the packets over 10GbE to the switch. Figure 5.2 gives the block diagram of the FPGA design. The system utilizes 


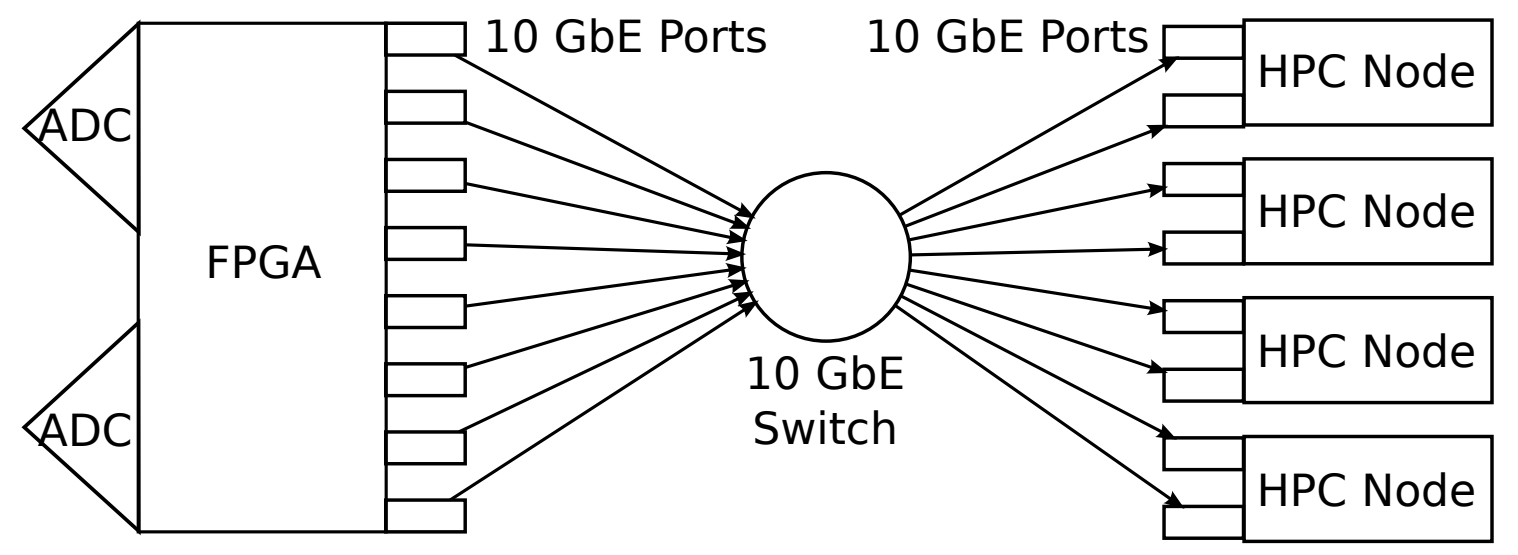

Figure 5.1: The SERENDIP VI system architecture. The FPGA is a ROACH II board with two 5 Gsps ADC boards, one for each polarization. Packetized coarse spectra are sent over eight $10 \mathrm{GbE}$ ports to the Ethernet switch, where it is distributed to the HPC nodes equipped with GPUs.

two 5 Gsps ADC boards, one each for a polarization channel, that digitize data to 8 bits. A streaming 1024-point Fast Fourier Transfrom (FFT) stage, preceded by a pre-filter stage, implements the Polyphase Filter Bank (PFB) technique, to achieve better isolation between channels. This provides the design some amount of resilience against radio frequency interference. The data at the output of the PFB stage is scaled appropriately and re-quantized to 8 bits, before the two polarizations are interleaved and packetized. The packetizer packs the interleaved dual-polarization complex samples into Ethernet frames and passes it to the $10 \mathrm{GbE}$ transmission block that uses the eight $10 \mathrm{GbE}$ links of the ROACH-II to send data to the switch. Each 10GbE link carries one-eighth of the total number of channels, in this case, 64 channels per packet, that correspond to a bandwidth of $625 \mathrm{MHz}$.

Figure 5.3 gives the SERENDIP VI packet format. The packet is an Ethernet jumbo frame with 8192 bytes of data in the payload section, in addition to an 8-byte header that carries the frame count and spectrum segment identifier. Each packet 


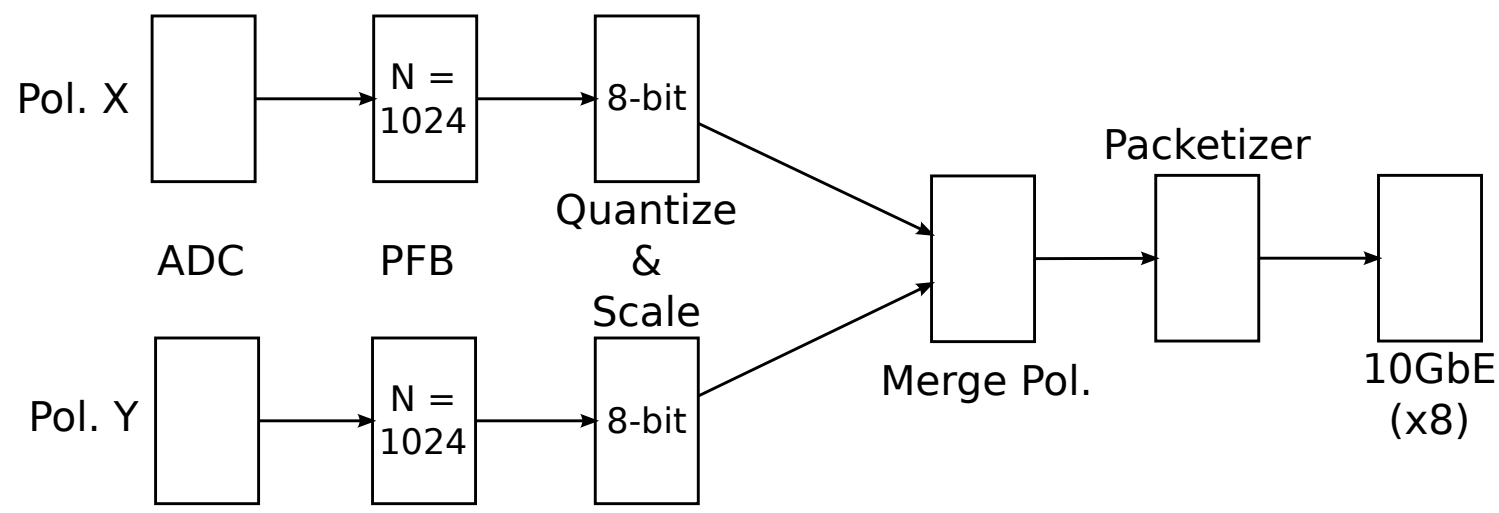

Figure 5.2: SERENDIP VI FPGA design. Data from each 5 Gsps ADC board are channelized into 1024 coarse channels using a PFB, and scaled and re-quantized to 8 bits. Data from the two polarizations are then merged, packetized (while splitting it to eight 64-channel blocks), and transmitted over eight 10GbE links.

\begin{tabular}{|c|c|c|c|c|c|c|c|}
\hline \multicolumn{7}{|c|}{ Frame Count } & \multirow{2}{*}{$\begin{array}{c}\text { Spectrum } \\
\text { Segment } \\
\mathrm{S}_{0}-\mathrm{c}_{1}-\mathrm{p}_{1}-\mathrm{im}\end{array}$} \\
\hline$s_{0}-c_{0}-p_{0}-r e$ & $s_{0}-c_{0}-p_{0}-i m$ & $s_{0}-c_{0}-p_{1}-r e$ & $s_{0}-c_{0}-p_{1}-i m$ & $s_{0}-c_{1}-p_{0}-r e$ & $s_{0}-c_{1}-p_{0}-i m$ & $s_{0}-c_{1}-p_{1}-r e$ & \\
\hline$S_{0}-C_{2}-p_{0}-r e$ & $\mathrm{~S}_{0}-\mathrm{C}_{2}-\mathrm{p}_{0}-\mathrm{im}$ & $s_{0}-c_{2}-p_{1}-r e$ & $S_{0}-C_{2}-p_{1}-i m$ & $s_{0}-c_{3}-p_{0}-r e$ & $\mathrm{~S}_{0}-\mathrm{c}_{3}-\mathrm{p}_{0}-\mathrm{im}$ & $s_{0}-c_{3}-p_{1}-r e$ & $\mathrm{~S}_{0}-\mathrm{C}_{3}-\mathrm{p}_{1}-\mathrm{im}$ \\
\hline$s_{0}-c_{4}-p_{0}-r e$ & $\mathrm{~S}_{0}-\mathrm{C}_{4}-\mathrm{p}_{0}-\mathrm{im}$ & $s_{0}-c_{4}-p_{1}-r e$ & $\mathrm{~S}_{0}-\mathrm{C}_{4}-\mathrm{p}_{1}-\mathrm{im}$ & $s_{0}-c_{5}-p_{0}-r e$ & $s_{0}-C_{5}-p_{0}-i m$ & $s_{0}-c_{5}-p_{1}-r e$ & $s_{0}-C_{5}-p_{1}-i m$ \\
\hline$s_{0}-c_{6}-p_{0}-r e$ & $s_{0}-c_{6}-p_{0}-i m$ & $s_{0}-c_{6}-p_{1}-r e$ & $s_{0}-c_{6}-p_{1}-i m$ & $s_{0}-c_{7}-p_{0}-r e$ & $s_{0}-c_{7}-p_{0}-i m$ & $s_{0}-c_{7}-p_{1}-r e$ & $s_{0}-C_{7}-p_{1}-i m$ \\
\hline$s_{1}-c_{0}-p_{0}-r e$ & $s_{1}-c_{0}-p_{0}-i m$ & $s_{1}-c_{0}-p_{1}-r e$ & $s_{1}-c_{0}-p_{1}-i m$ & $s_{1}-c_{1}-p_{0}-r e$ & $s_{1}-c_{1}-p_{0}-i m$ & $s_{1}-c_{1}-p_{1}-r e$ & $s_{1}-c_{1}-p_{1}-i m$ \\
\hline \multicolumn{8}{|c|}{$\cdots$} \\
\hline$S_{255}-c_{6}-p_{0}$-re & $s_{255}-c_{6}-p_{0}-i m$ & $\mathrm{~S}_{255}-\mathrm{C}_{6}-\mathrm{p}_{1}$-re & $\mathrm{S}_{255}-\mathrm{C}_{6}-\mathrm{p}_{1}-\mathrm{im}$ & $\mathrm{S}_{255}-\mathrm{C}_{7}-\mathrm{p}_{0}$-re & $s_{255}-c_{7}-p_{0}-i m$ & $\mathrm{~S}_{255}-\mathrm{C}_{7}-\mathrm{p}_{1}$-re & $S_{255}-C_{7}-p_{1}-i m$ \\
\hline
\end{tabular}

Figure 5.3: SERENDIP VI UDP packet payload format. Each UDP payload contains 8200 bytes, with an eight-byte header, and 8192 bytes of data.

contains eight channels out of the 64 that is intended for one computing node. The data transport pipeline in the HPC node stores these packets in a shared memory ring buffer, for the benefit of the data processing code. 


\subsection{Data Transport Framework}

The data transport framework is a software component of the system that reads data from a $10 \mathrm{GbE} \mathrm{NIC} \mathrm{on} \mathrm{the} \mathrm{HPC} \mathrm{node} \mathrm{and} \mathrm{transports} \mathrm{that} \mathrm{data} \mathrm{through} \mathrm{the} \mathrm{pro-}$ cessing pipeline, finally writing the reduced data to disk. Figure 5.4 shows the data flow diagram for the SERENDIP VI data transport framework. This framework is based on, and is a modification of that used in the Versatile GBT Astronomical Spectrometer ${ }^{2}$ (VEGAS). The data transport framework has four computing threads, namely, the network thread, the GPU thread, the CPU thread, and the disk thread. The network thread uses UNIX sockets to receive the UDP data from the Ethernet switch. It extracts the SERENDIP VI header and payload from the UDP packet and writes it to a shared memory ring buffer. The GPU thread reads data from the ring buffer, and performs fine channelization. The GPU thread is discussed in detail in $\S 5.5$. Once spectra are ready, the GPU thread writes these to the next shared memory ring buffer, from which the CPU thread reads data. The CPU thread performs further accumulation of spectra as needed, and also performs baseline smoothing and thresholding to identify strong candidates. It writes out the candidate list to the following ring buffer. The disk thread writes this data to disk.

To improve performance, the processor affinity for each thread is set during initialization. The SMP affinity of the interrupt request of the NIC is set manually during installation. Together, this reduces the overhead involved in swapping tasks into and out of the processor.

\footnotetext{
${ }^{2}$ http://www.gb.nrao.edu/vegas/
} 


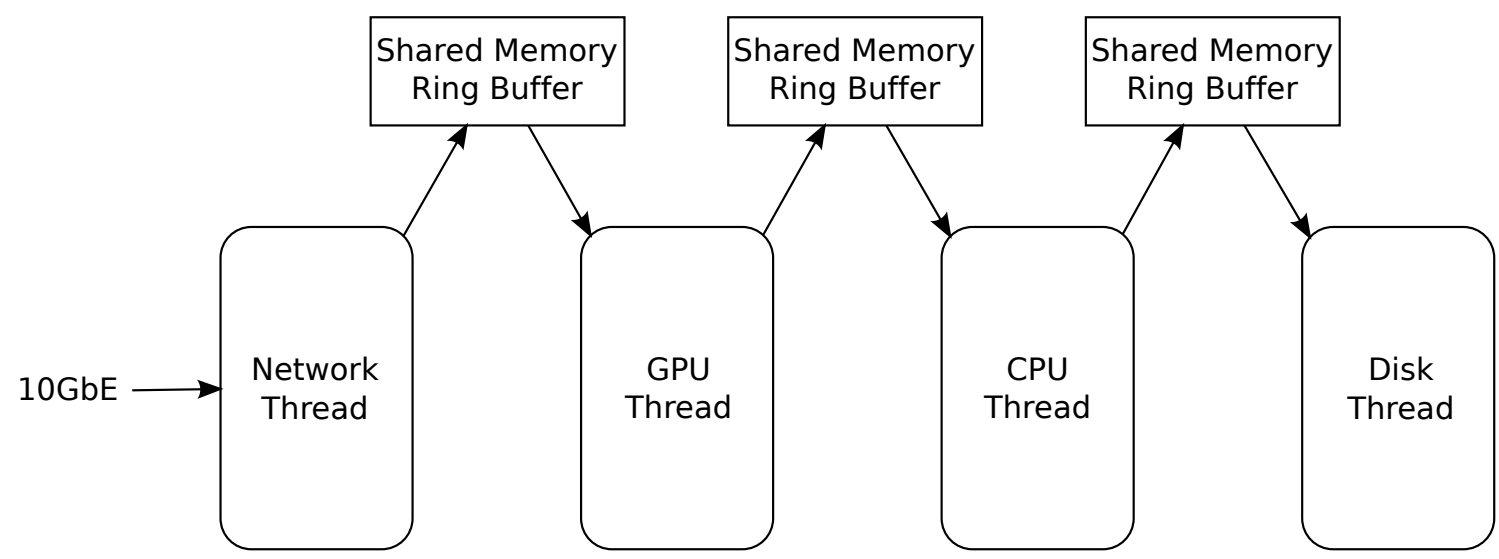

Figure 5.4: The SERENDIP VI data transport framework. The computing threads form a data processing pipeline, with data being moved from the network thread to the disk thread via a series of shared memory ring buffers.

\subsection{GPU Spectrometry}

Each instance of the GPU program processes the 64-channel data from the FPGA board. The input data is dual-polarization, 8-bit, interleaved, complex values. The GPU code takes these samples and performs FFT using the CUDA FFT library (CUFFT). The spectrometer outputs $X^{2}, Y^{2}, \Re\left(X Y^{*}\right)$, and $\Im\left(X Y^{*}\right)$, where $X$ and $Y$ are the magnitude spectra of the two polarizations. Note that all the Stokes parameters can be generated from these output samples. The high-level algorithm for the GPU code is as follows:

1. Initialization: Create FFT plan, allocate memory

2. Copy data from host (CPU) to device (GPU)

\section{Perform FFT}

4. Accumulate spectra for desired duration

5. Copy spectra from device to host 


\subsubsection{Handling Long Transforms}

The high bandwidth and high spectral resolution requirements of SERENDIP VI means that long transforms need to be performed on the GPU. With a bandwidth of $312.5 \mathrm{MHz}$ per polarization per pipeline, and a $\sim 1 \mathrm{~Hz}$ resolution, the length of FFT required would be 268435456 . With four bytes for each time sample in the input, an FFT of such a length would require $1 \mathrm{~GB}$ of memory just for the input, or 2 GB of memory for an out-of-place transform. Together with other memory requirements, this can be more memory than present in most commercial gaming GPUs. To work around this problem we perform a 'batched FFT' in which FFT is performed only for a subset of the 64 channels at any given time. For instance, performing FFT for only 32 channels at a given time halves the memory required.

\subsection{Conclusion}

SERENDIP VI is under active development. The Arecibo version of the instrument is planned for completion in the spring of 2014, and the GBT version is planned for late 2014. Installation and preliminary tests in Arecibo were carried out in February 2014 .

The instrument has been tested for functionality using simulated data. Figure 5.5 shows the spectrum of a simulated data input. 

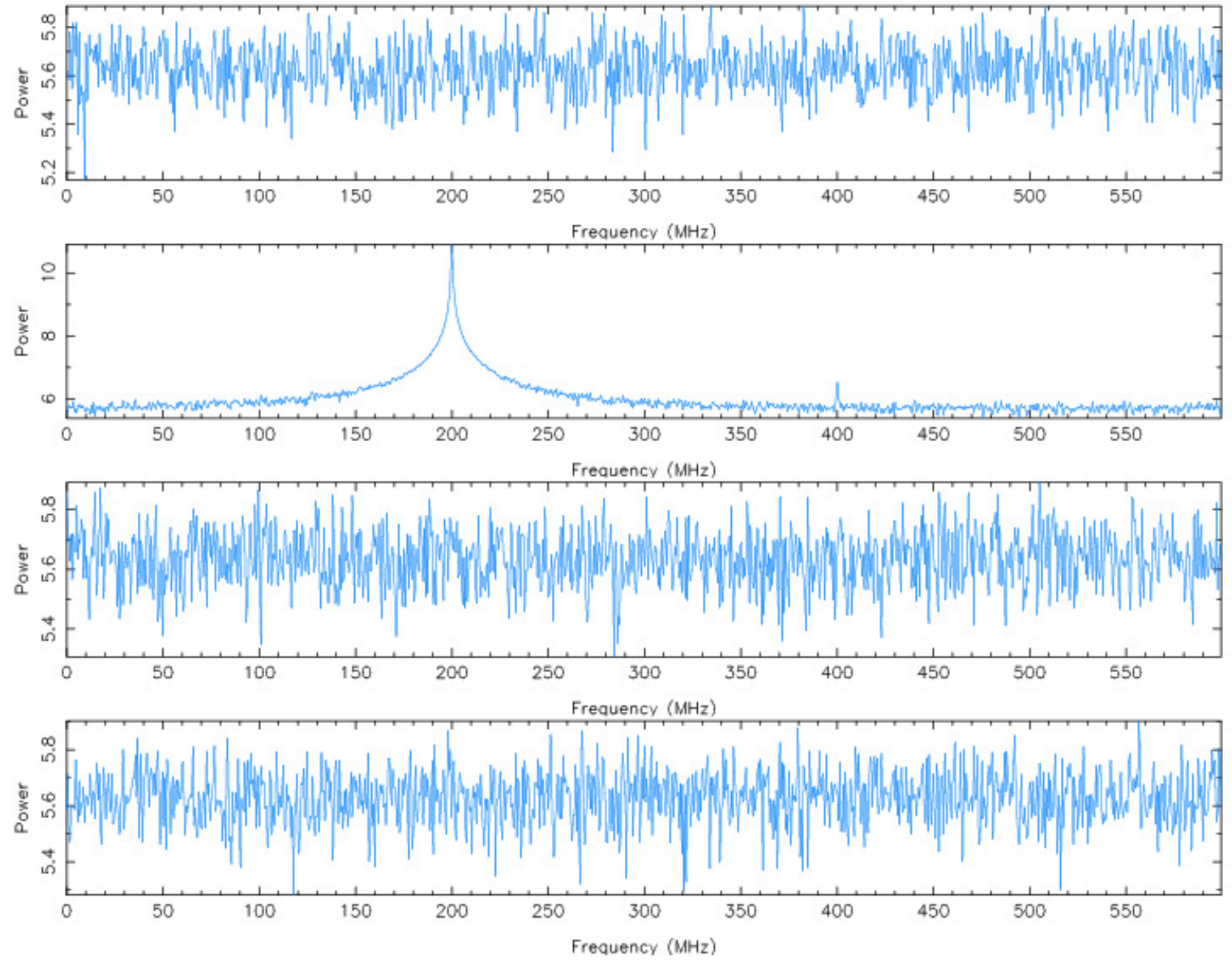

Figure 5.5: Output plot for a standalone version of the SERENDIP VI GPU code, using simulated data. Each panel represents a different signal channel/sub-band. The simulated signal, a $200 \mathrm{MHz}$ tone, is present in only the second sub-band. 


\section{Chapter 6}

\section{Jumping the energetics queue: Modulation of pulsar signals by extraterrestrial civilizations}

\subsection{Introduction}

The Kardashev scale (Kardashev, 1964) classifies civilizations according to their ability to consume energy. The human civilization is the prototypical Kardashev Type-I civilization ${ }^{1}$, consuming energy at the rate of $\sim 4 \times 10^{19} \mathrm{erg} \mathrm{s}^{-1}$. A Kardashev Type-II civilization consumes $\sim 4 \times 10^{33} \mathrm{erg} \mathrm{s}^{-1}$ - equivalent to the energy output of a Sun-like star. A Type-III civilization would be capable of consuming $\sim 4 \times 10^{44}$ $\operatorname{erg} \mathrm{s}^{-1}$, which is of the order of the luminosity of galaxies. Any radio beacon that an extraterrestrial intelligence (ETI) builds would necessarily have a transmission power not more than what that civilization consumes. In this chapter, we assume that the transmission power of an ETI beacon is of the same order of magnitude as their energy consumption. Following Kardashev (1964), we can calculate the power required to isotropically transmit a signal with a bandwidth $\Delta f$ across the Galaxy, such that it can be received at an Arecibo-like radio telescope with a signal-to-noise ratio $S / N$, as

$$
P \approx 6.6 \times 10^{24}\left(\frac{\Delta f}{\mathrm{~Hz}}\right)\left(\frac{S / N}{10}\right) \mathrm{erg} \mathrm{s}^{-1}
$$

\footnotetext{
${ }^{1}$ The Kardashev scale has been redefined and expanded by others (see, for example, Horowitz \& Sagan, 1993), but in this chapter, we follow the original definition presented in Kardashev (1964), with no consequence to our treatment.
} 
Traditional radio SETI experiments search for narrow-band $(\sim 1 \mathrm{~Hz})$ signals. Even for such narrow-band signals, a detectable $S / N$ would imply a transmission power that can be generated only by civilizations that are much more advanced than TypeI. The main drawback of using narrow-band signals as beacons is that the ETI is forced to choose some special frequency that may not be monitored by potential receivers. The solution to this problem is to transmit over a larger bandwidth, but since $P \propto \Delta f$, the power requirement increases. For instance, for $\Delta f=1 \mathrm{GHz}$, $P \sim 10^{33} \mathrm{erg} \mathrm{s}^{-1}$, which can only be produced by civilizations that are at least Type-II. Less advanced civilizations, however, can work around this problem by making use of appropriate naturally-occurring radio transmitters. In this chapter, we propose that an ETI that is moderately more advanced than humans but not yet achieving a higher Kardashev type, may be able to use radio pulsars as sources of power at levels otherwise unachievable, modulating the broad-band pulsar signal for communication. The minimum requirement for such an endeavour would only be the ability to build and launch a modulating satellite to a nearby pulsar.

A pulsar is a neutron star that emits coherent radio radiation from its magnetic poles (see Lorimer \& Kramer, 2005). Pulsars are fast-rotating, and usually detected due to the fact that an offset exists between their magnetic and rotational axes, causing them to appear as periodic signals, with an observer typically receiving one pulse per one complete rotation of the pulsar. The radio luminosity of a pulsar with spin period $P$ situated at a distance $d$ from an observer is given in terms of the 
measured flux density as

$$
L=\frac{4 \pi d^{2}}{\delta} \sin ^{2}\left(\frac{\rho}{2}\right) \int_{f_{1}}^{f_{2}} S_{\text {mean }}(f) d f
$$

where $\delta=W_{\text {eq }} / P$ is the pulse duty cycle ( $W_{\text {eq }}$ is the equivalent pulse width), $\rho$ is the radius of the pulsar emission cone, the integrand is the mean flux density of the pulsar as a function of frequency $f$, and $f_{1}$ and $f_{2}$ bound the spectral range of the observation. Using typical values of $\delta$ and $\rho$, a pulsar with $P=1$ s situated at a distance of $1 \mathrm{kpc}$, with a measured $1400 \mathrm{MHz}$ flux density of $1 \mathrm{mJy}$, would have a radio luminosity $\approx 7.4 \times 10^{27} \mathrm{erg} \mathrm{s}^{-1}$. On the Kardashev scale, such a pulsar would therefore correspond to a beacon produced by a civilization between Type-I and Type-II. We speculate that a civilization with the minimum capability of sending a spacecraft to a nearby pulsar to install an orbital modulator for the sweeping pulsar beam would be able to harness the energy emission of pulsars without actually building and operating a transmitter so powerful (or being capable of doing so).

Previous works have considered extraterrestrial civilizations making use of naturally-occurring phenomena to announce their presence to any listeners. For example, Cordes (1993) has suggested that extraterrestrial civilizations may make use of astrophysical masers to amplify engineered signals, thereby transmitting more power than their position on the Kardashev scale might allow them to. A critical drawback of using a maser-based communication system is that masers are usually directional, and hence require the transmitter and receiver to be serendipitously aligned. Pulsar beams, on the other hand, albeit directional, are swept around due 
to the rotation of the star, thereby covering a much larger area of the sky, increasing the probability of detection. A system that makes use of pulsars, in addition to being used as beacons, can also be configured for directional communication, with say, a distant spacecraft or planetary system. Fabian (1977) and Corbet (1997) have discussed the possibility of generating X-ray pulses by dropping matter onto the surface of a neutron star, or modulating the X-ray emission of accreting neutron stars. Learned et al. (2008) has proposed that ETI may modulate the period of Cepheid variables to achieve signaling, by triggering pulsations using neutrinos beamed to the stellar core.

Cordes \& Sullivan (1995) and Sullivan \& Cordes (1995) postulate that ETI would employ 'astrophysical coding' - i.e., transmitting signals that can be detected using astrophysical signal analysis - in beacons. They argue that such a signal is more likely to be detected because astronomers would be able to easily analyse it. The idea proposed in this chapter is a kind of astrophysical coding technique and enjoy the benefit of higher likelihood of detectability.

The outline of this chapter is as follows: In $\S 6.2$, we describe our proposed modulation mechanism, and in $§ 6.3$, we discuss the information content of the beacon. In $\S 6.4$, we discuss potential observational signatures of artificial modulation, and in $\S 6.5$, we analyse energy considerations for this signalling scheme, before concluding in $§ 6.6$. 


\subsection{Modulation mechanism}

Installing a modulator on a pulsar would require considerations of the emission geometry of the pulsar being engineered. If we assume an inclination angle $\alpha=90^{\circ}$ (i.e., the magnetic axis orthogonal to the spin axis), the modulating satellite could orbit synchronously with the pulsar spin period to allow the signal to be transmitted over the entire area of the sky covered by the pulsar beam. In the more typical case of non-orthogonal axes, a polar orbit in which the satellite intersects the pulsar beam periodically would result in directional transmission. A scaffolding shell around the pulsar in which modulating elements are placed at locations where the pulsar beam intersects with the scaffold would result in the ability to cover the entire beaming solid angle of the pulsar.

We first consider a toy model of an orbital modulator that is synchronous with the pulsar rotation, assuming that the inclination angle of the pulsar beam, $\alpha=90^{\circ}$, as shown in Figure 6.1(a). For a pulsar with mass $M$ and period $P$, equating centripetal acceleration to the acceleration due to gravity gives an orbital radius

$$
r \approx 1.7 \times 10^{3}\left(\frac{M}{1.4 M_{\odot}}\right)^{1 / 3}\left(\frac{P}{\mathrm{~s}}\right)^{2 / 3} \mathrm{~km}
$$

For a canonical $1.4 M_{\odot}$ pulsar with $P=1 \mathrm{~s}$, this gives $r \approx 1700 \mathrm{~km}$, with a tangential velocity component of approximately $4 \%$ the speed of light. To probe the structural integrity of the satellite at this distance, we model the satellite as a solid steel cylindrical bar $10 \mathrm{~m}$ in length and $1 \mathrm{~m}$ in radius, oriented in such a way that the long axis is directed radially outwards from the pulsar. The elongation of the bar 
(a)

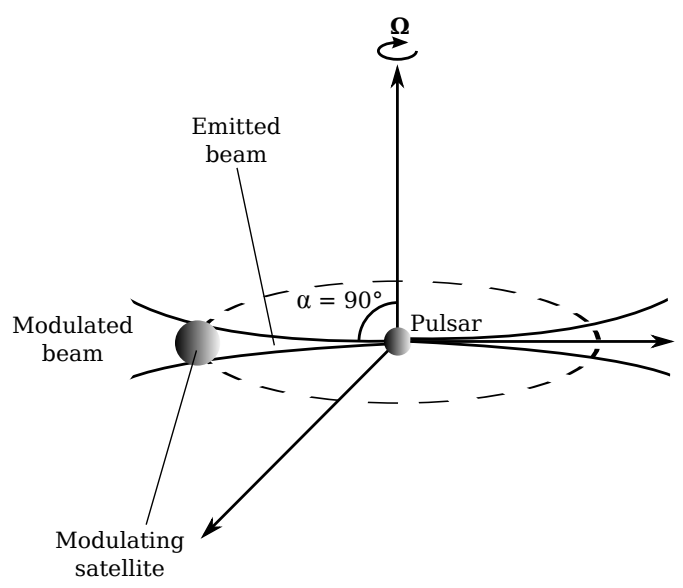

(b)

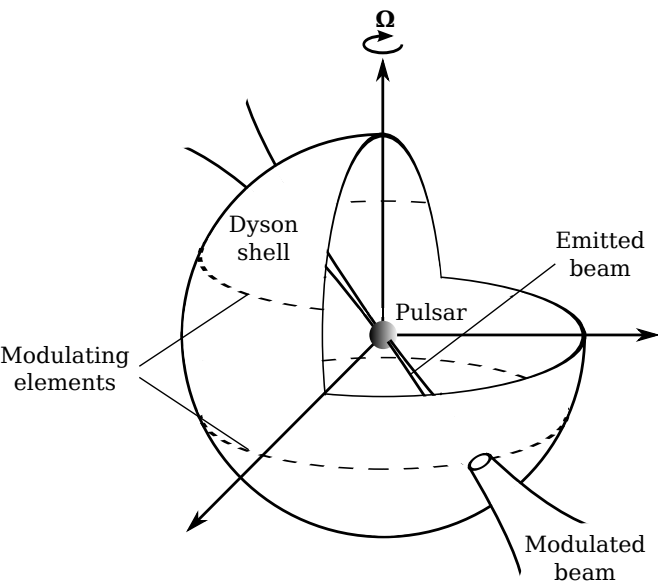

Figure 6.1: (a) Schematic of a modulating satellite co-rotating with a pulsar that has an inclination angle $\alpha=90^{\circ}$; (b) Cross-section of a Dyson shell around a pulsar. Modulating elements are placed along the loci of the pulsar beams on the shell.

due to the differential gravity on either of its ends is on the order of $10^{-5} \mathrm{~m}$, and therefore, is inconsequential.

Instead of a satellite, a civilization capable of advanced astronomical engineering could build an equatorial ring around the pulsar that covers the entire area swept by the beam. A less desirable option would be to have a satellite in a nonsynchronous orbit periodically intercepting the pulsar beam, but this would severely reduce the beaming fraction of the modulated beam, and also make message reconstruction more difficult.

The typical case of non-orthogonal beams, however, is more complicated. A modulator in a polar orbit that intercepts the pulsar beams periodically could be built, but this has the problem of low beaming fraction, which would not serve as a beacon, but could be used for directional communication. For a beacon, the last option - albeit one that would require a significant amount of astronomical engineering - would be a scaffold around a pulsar akin to a Dyson sphere (Dyson, 1960), 
with modulating elements placed at the points where the pulsar beam intersects the scaffold, as shown in Figure 6.1(b). Traditional Dyson structures provide generalpurpose energy for the consumption of an advanced civilization. In this case, the energy of the host star is used only for producing a detectable beacon. The materials that make up the scaffold, and the structural engineering of the scaffold should be such that it should not interact with the particles and field lines within the pulsar magnetosphere, except at the modulating elements. The radius of the shell should be large enough to accommodate the pulsar emission region. Kijak \& Gil (2003) give a semi-empirical formula to calculate the heights of emission regions of pulsars, which, for a $10 \mathrm{~km}$-radius pulsar, is

$$
r_{\mathrm{em}} \approx 400\left(\frac{f}{\mathrm{GHz}}\right)^{-0.26}\left(\frac{\dot{P}}{10^{-15}}\right)^{0.07}\left(\frac{P}{\mathrm{~s}}\right)^{0.30} \mathrm{~km}
$$

where $f$ is the frequency of radio emission at $r_{\mathrm{em}}, \dot{P}$ is the period derivative, and $P$ is the period of the pulsar. Assuming that the lowest frequency of interest to the modulation is $10 \mathrm{MHz}$, a pulsar with $P=1 \mathrm{~s}$ and $\dot{P}=10^{-15} \mathrm{~s} \mathrm{~s}^{-1}$ would have a maximum emission height of interest of $\sim 1300 \mathrm{~km}$. This gives the minimum radius of the Dyson sphere.

As in any communication system, the modulation could be one of many types. It could be amplitude modulation, frequency modulation, or phase modulation, either analogue or digital. In this chapter, we consider the simplest case, where an amplitude modulator toggles between 0\% modulation (modulator transparent to pulsar radiation) and $100 \%$ modulation (modulator quenches entire emission) to 
achieve preferential nulling, resulting in single-bit data transmission. For the sake of simplicity, we also assume that the nulling is frequency-independent, that is, during a null, the entire radio emission of the pulsar is quenched. The information content of this system is discussed in $\S 6.3$.

We do not speculate on the actual nature of the modulator as it would most likely be based on technology not yet invented by humans, although it would seem that the signal-modulating mechanism could be based on confined plasma, or perhaps, electro-optic modulators (see Purvinis \& Maldonado, 2010). In the case of nulling modulation, the modulating material would need to scatter, absorb or redirect the entire radiation falling on it. If the radiation is absorbed, this would manifest as an increase in the temperature of the modulator and could show up as thermal radiation when the energy is re-radiated. This is discussed in more detail in $§ 6.4$.

\subsection{Information content}

A single-bit modulation system as mentioned in the previous section would support only low bit rates. For the nulling method, the data rate, $R=1 / P$ bits per second, where $P$ is the spin period of the pulsar in seconds. Even utilizing the fastest pulsars, the transmission rate would be less than $1 \mathrm{Kbps}$. In this model, we have assumed that the nulling is independent of frequency. A more complex system could make use of frequency-dependent nulling, thereby increasing the data rate of the signal. Further increase in data rate using amplitude modulation would require more complex modulation mechanisms wherein the amplitude of a pulse varies over 
a range of modulation depths. Another possibility is using a modulating signal whose frequency is much larger than the pulsar period (but less than the 'carrier' frequency, or the radio frequency). This would manifest as narrow features in the time domain, within the on-pulse of the pulsar.

The artificially-modulated pulsar signal contains another piece of 'information' that is astrophysically-coded - the fact that the ETI identified the neutron star that was engineered as a pulsar indicates that their civilization may be based on at least one planet that is within the beaming solid angle of the pulsar. If the inclination angle of the pulsar beam can be determined, this helps derive a coarse constraint on the location of the civilization.

\subsection{Observable effects}

Pulse nulling (Backer, 1970) is observed in many pulsars, and is usually attributed to changes in the plasma currents in the pulsar magnetosphere (see, for example, Wang et al., 2007), although this explanation has not been conclusively established. Statistical studies of nulling and the possibly related phenomena of mode-changing and drifting sub-pulses have the potential to determine any sign of non-natural processes in action. Redman \& Rankin (2009) treated the pulse-null stream for a set of pulsars as a binary sequence and performed a statistical runs test, and found that nulling is not random in many pulsars that exhibit the phenomenon. An artificially-nulled pulsar would also show up as non-random in such an analysis, but in general, it is unclear how to distinguish between artificial and natural nulling. If 
we assume that the intention of the modulation is to serve as a beacon, one of the easiest ways to display an artificial nature would be to have the null runs last for prime numbers of rotations. An example histogram of null-run duration is shown in Figure 6.2, which is extremely unlikely to be produced due to any natural process. Another way would be to null a pulse once after every $n$ complete rotations of the pulsar, where $n$ is a prime number. This system might be preferable if nulling the pulsar is expensive in terms of energy, as each null run lasts for only one complete rotation of the pulsar. In this case, a histogram for the number of rotations between nulls would look similar to Figure 6.2. Any other information the ETI would like to transmit could additionally be imposed as amplitude modulation on the non-nulled pulses.

Irrespective of whether the modulation is due to an orbiting satellite or due to a Dysonian scaffold, during a null, if the pulsar signal is absorbed, the temperature of the absorbing medium (modulating element) should increase. To prevent heat buildup, the modulating element will need to shed this excess energy in a timescale of the duration of one complete rotation of the pulsar. Looking for excess emission with a thermal spectrum during the null phases of a pulsar would indicate such a process in action. Considering the case of an orbiting satellite as shown in Figure 6.1(a), assuming that the power emitted by the pulsar is given by the spin-down luminosity $\dot{E}$ and that the modulating element is in thermal equilibrium, the temperature of 


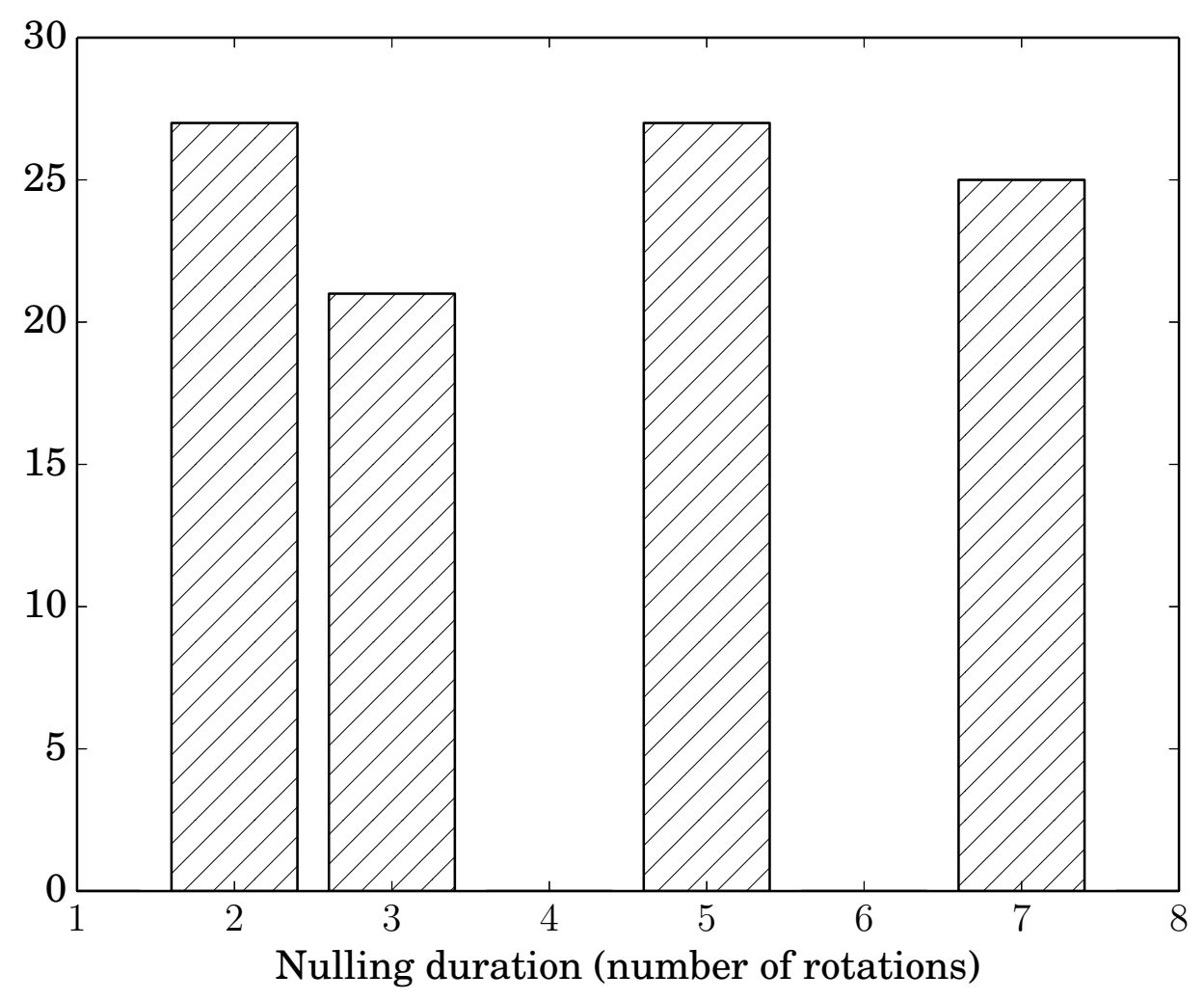

Figure 6.2: Example histogram of null duration in terms of number of rotations of the pulsar. This histogram is indicative of a non-natural nulling process. 
this secondary emission is

$$
T \approx 1.1 \times 10^{6}\left(\frac{r}{\mathrm{~km}}\right)^{-1 / 2}\left(\frac{\dot{P}}{10^{-15}}\right)^{1 / 4}\left(\frac{P}{\mathrm{~s}}\right)^{-3 / 4} \mathrm{~K}
$$

where $r$ is the orbital radius. For a pulsar with $P=1 \mathrm{~s}$ and $\dot{P}=10^{-15} \mathrm{~s} \mathrm{~s}^{-1}$, and taking $r=1700 \mathrm{~km}$ as derived in $\S 6.2$, we get $T \approx 2.7 \times 10^{4} \mathrm{~K}$, which corresponds to a wavelength of approximately $107 \mathrm{~nm}$, in the ultraviolet. An excess of thermal emission that peaks in the ultraviolet during the null phases of this pulsar, therefore, would indicate the presence of an absorbing medium.

Pulsar signals are affected by the cold plasma that makes up the interstellar medium (ISM) in various ways (Rickett, 1990; Cordes, 2002a). A challenging aspect of detecting an intelligent signal within the pulsar beam is decoupling the modulation and the effects of the ISM, particularly for pulsars in the strong scattering regime. A simple-minded approach would be to assume that the ETI-imposed nulling covers the entire band of radio emission from the pulsar, whereas diffractive scintillation is frequency-dependent. Another factor that helps discriminate between artificial modulation and scintillation is the difference in time scales. For a typical pulsar with a transverse velocity $v=10^{5} \mathrm{~m} \mathrm{~s}^{-1}$, exhibiting diffractive scintillation due to inhomogeneities in the ISM of the size scale $r_{\mathrm{d}}=10^{7} \mathrm{~m}$, the timescale of scintillation is given by $t_{\mathrm{d}}=r_{\mathrm{d}} / v=100 \mathrm{~s}$ (see Narayan, 1992). This timescale is longer than any variation due to artificial modulation, which is on the order of the pulse period. 


\subsection{Discussion}

The decision of using a conventional radio transmitter vis-à-vis a pulsar-based beacon depends on the number of pulsars required to cover the entire sky, the cost of installing modulators around those pulsars, and the lifetime of the modulating system. Assuming that the beaming solid angle of a pulsar is about $20 \%$ of the sky, it would take at least five such pulsars to cover the entire sky. If the energy requirements for sending modulating satellites to those pulsars (or building modulating Dyson shells around those pulsars) is less than that of building and operating a perpetual, omnidirectional, conventional transmitter of the same power, the former would be the optimal solution, provided the lifetime of such a system is long enough.

A spacecraft that has to be inserted into orbit around another star has to accelerate to some maximum velocity and then decelerate. To first order, the energy required for this is twice the kinetic energy of the spacecraft, $E=m c^{2}(\gamma-1)$, where $m$ is the mass of the spacecraft (including fuel mass) and $\gamma=1 / \sqrt{1-v^{2} / c^{2}}$ is the Lorentz factor, where $v$ is final velocity of the spacecraft. Assuming a spacecraft mass of $10^{9} \mathrm{~kg}$, travelling at a constant velocity equal to $10 \%$ of the speed of light, taking a time $t$ to travel a distance equal to the minimum Earth-pulsar distance for reported values in the ATNF Pulsar Catalogue 2 (160 pc; Manchester et al., 2005), the average energy consumption rate is approximately given by $E / t \sim 10^{19} \mathrm{erg}$ $\mathrm{s}^{-1}$. Since this is much less than the energy output of a pulsar, the cost involved

\footnotetext{
${ }^{2}$ http://www.atnf.csiro.au/people/pulsar/psrcat/
} 
in installing a few such satellites is negligible compared to the transmission power achieved. The major factor determining feasibility is then the lifetime of the system. For a modulating satellite with no attitude stabilization, the lifetime depends on two factors: (a) the radiation pressure exerted by the pulsar beam and (b) the pressure due to particles that follow magnetic field lines impinging on the satellite. These two effects combine to push the satellite out of its orbit. For a modulating satellite with attitude control, the lifetime would depend on the amount of fuel it can carry. It is conceivable that electrical energy for attitude correction can be extracted from the pulsar beam itself, in which case, the lifetime will be considerably longer, with an upper limit given by the radio lifetime of the pulsar, which is about $10^{7} \mathrm{yr}$ for normal pulsars or about $10^{9} \mathrm{yr}$ for millisecond pulsars.

For a Dyson shell, the factors affecting feasibility are different. The cost incurred in installation would be much higher than that of a modulating satellite. The lifetime would depend on the structural properties of the scaffold, and also whether the surrounding environment of the pulsar contains potentially destructive asteroids or other debris.

There is one caveat to the pulsar modulation scheme discussed in this chapter, namely, that we ignore the rate of increase of energy consumption of the extraterrestrial civilization. If the time it takes to advance to a higher developmental stage one at which the ETI can afford to build a beacon matching typical pulsar luminosity - is less than that for their spacecraft to reach the target pulsars, the best choice for the civilization would be to wait. It is hard to predict rates of development, and depending on the availability of interstellar travel technology and nearby pulsars, 
civilizations may or may not choose to implement this scheme. For instance, in the event that intelligent, technological life evolved on a planet orbiting a pulsar, or one orbiting a companion to a pulsar in a multiple-star system, it would not only be energetically favourable, but quicker, to implement a pulsar modulation scheme.

\subsection{Conclusion}

It is reasonable to assume that energy production/consumption goes hand-in-hand with the development of technological civilizations, as seen on Earth. Technological civilizations should, therefore, sooner or later, embark on large-scale energy harvesting endeavours, such as building Dyson spheres. Even though it is unclear how inclined a civilization would be to announce their presence explicitly using beacons, if we assume that they are so inclined, modulating the signal of a nearby pulsar would be one of the most energy-efficient ways of doing it. Building a Dysonian scaffold around a pulsar would cost much less in terms of material than building a Dyson sphere at a habitable distance around a Sun-like star, and would also be an engineering proof-of-concept for a pre-Kardashev-Type-II civilization.

Statistical studies of pulsar emission, such as those of nulling and pulse-topulse and intra-pulse intensity variation, have the potential to discover non-natural processes in action, thereby indicating the presence of technologically advanced civilizations in the Galaxy. Single-pulse observations of pulsars using radio telescopes with large collecting areas will provide the high-quality data required for this purpose. 


\section{Chapter 7}

\section{Conclusion}

\subsection{Instrumentation for transient search/studies}

The Graphics Processing Unit (GPU) has become an integral part of astronomical instrumentation, enabling high-performance online data reduction and accelerated online signal processing. In Chapters 4 and 5 we described a wide-band reconfigurable spectrometer and a SETI back-end built using off-the-shelf GPU cards.

The GPU code behind the VEGAS spectrometer, when configured to use a polyphase filter bank (PFB), supports a dual-polarization bandwidth of up to 600 $\mathrm{MHz}$ (or a single-polarization bandwidth of up to $1.2 \mathrm{GHz}$ ). Without the PFB, the spectrometer supports a dual-polarization bandwidth of up to $800 \mathrm{MHz}$ (or a single-polarization bandwidth of up to $1.6 \mathrm{GHz}$ ). The benchmarking was done on an NVIDIA GeForce GTX 580 GPU card. The performance of the code can be increased by using newer generation dual-GPU cards, and also potentially with algorithm-level and code-level optimizations.

SERENDIP VI is a SETI spectrometer that is heterogeneous in architecture. Unlike VEGAS in which the FPGA sends out time samples for 8 sub-bands to the GPU, SERENDIP VI supports up to 64 sub-bands. It is also a high resolution instrument $(\sim 1 \mathrm{~Hz})$. The Arecibo version of the spectrometer is planned for completion in the spring of 2014, and the GBT version in late 2014. 


\subsection{Population statistics of globular cluster pulsars}

Studies of the Galactic population of radio pulsars have shown that their luminosity distribution appears to be log-normal in form. In Chapter 2, we investigated some of the consequences that occur when one applies this functional form to populations of pulsars in globular clusters. We used Bayesian methods to explore constraints on the mean and standard deviation of the luminosity function, as well as the total number of pulsars, given an observed sample of pulsars down to some limiting flux density, accounting for measurements of flux densities of individual pulsars as well as diffuse emission from the direction of the cluster. We applied our analysis to Terzan 5, 47 Tucanae and M 28, and demonstrated, under reasonable assumptions, that the number of potentially observable pulsars should be within $95 \%$ credible intervals of $147_{-65}^{+112}, 83_{-35}^{+54}$ and $100_{-52}^{+91}$, respectively. Beaming considerations would increase the true population size by approximately a factor of two. Using non-informative priors, however, the constraints are not tight due to the paucity and quality of flux density measurements. Future cluster pulsar discoveries and improved flux density measurements would allow this method to be used to more accurately constrain

the luminosity function, and to compare the luminosity function between different clusters.

\subsection{The Galactic center pulsar population}

The recent discovery of a magnetar in the Galactic center region has allowed Spitler et al. to characterize the interstellar scattering in that direction. They find that the tem- 
poral broadening of the pulse profile of the magnetar is substantially less than that predicted by models of the electron density of that region. This raises the question of what the plausible limits for the number of potentially observable pulsars - i.e., the number of pulsars beaming towards the Earth - in the Galactic center are. In Chapter 3, using reasonable assumptions - namely, (i) the luminosity function of pulsars in the Galactic center region is the same as that in the field, (ii) the region has had a constant pulsar formation rate, (iii) the spin and luminosity evolution of magnetars and pulsars are similar, and (iv) the scattering in the direction of the Galactic center magnetar is representative of the entire inner parsec - we showed that the potentially observable population of pulsars in the inner parsec has a conservative upper limit of $\sim 200$, and that it is premature to conclude that the number of pulsars in this region is small. We showed that the observational results so far are consistent with this number. We predict that future deep surveys of the Galactic centre with the GBT may uncover $\leq 6$ pulsars (at the 1-sigma level).

\subsection{SETI}

It has been speculated that technological civilizations evolve along an energy consumption scale first formulated by Kardashev, ranging from human-like civilizations that consume energy at a rate of $\sim 10^{19} \mathrm{erg} \mathrm{s}^{-1}$ to hypothetical highly advanced civilizations that can consume $\sim 10^{44} \mathrm{erg} \mathrm{s}^{-1}$. Since the transmission power of a beacon a civilization can build depends on the energy it possesses, to make it bright enough to be seen across the Galaxy would require high technological advancement. 
In Chapter 6, we discussed the possibility of a civilization using naturally occurring radio transmitters - specifically, radio pulsars - to overcome the Kardashev limit of their developmental stage and transmit super-Kardashev power. This is achieved by the use of a modulator situated around a pulsar, that modulates the pulsar signal, encoding information onto its natural emission. We discussed a simple modulation model using pulse nulling and considerations for detecting such a signal. We found that a pulsar with a nulling modulator will exhibit an excess of thermal emission peaking in the ultraviolet during its null phases, revealing the existence of a modulator. 


\section{Bibliography}

Alpar, M. A., Cheng, A. F., Ruderman, M. A., Shaham, J., 1982, Nature, 300, 728

Antoniadis, J., et al., 2013, Science, 340, 448

Archibald, A. M., et al., 2009, Science, 324, 1411

Arkhypov, O. V., Rucker, H. O., 2009, A\&A, 496, 269

Armour, W., et al., 2012, in Ballester, P., Egret, D., Lorente, N. P. F., eds., ASP Conf. Ser. Vol. 461, Astronomical Data Analysis Software and Systems XXI, Astron. Soc. Pac., San Francisco, p. 33

Backer, D. C., 1970, Nature, 228, 42

Bagchi, M., Lorimer, D. R., Chennamangalam, J., 2011, MNRAS, 418, 477

Barsdell, B. R., Bailes, M., Barnes, D. G., Fluke, C. J., 2012, MNRAS, 422, 379

Bates, S. D., Lorimer, D. R., Verbiest, J. P. W., 2013, MNRAS, 431, 1352

Bates S. D., et al., 2011, MNRAS, 411, 1575

Bégin, S., 2006, Ph.D. Thesis, UBC

Bower, G. C., et al., 2014, ApJ, 780, L2

Bowyer, S., 2011, in Searching for Extraterrestrial Intelligence, The Frontiers Collection, Springer-Verlag Berlin Heidelberg, p. 99 
Boyles, J., Lorimer, D. R., Turk, P. J., Mnatsakanov, R., Lynch, R. S., Ransom, S. M., Freire, P. C., Belczynski, K., 2011, ApJ, 742, 51

Bracewell, R. N., 1999, The Fourier Transform and its Applications, McGraw-Hill

Buttu, M., et al., 2013, ATel \#5053

Camilo, F., Rasio, F. A., 2005, in Rasio, F. A., Stairs, I. H., eds, ASP Conf. Ser. Vol. 328, Binary Radio Pulsars. Astron. Soc. Pac., San Francisco, p.147

Camilo, F., Lorimer, D. R., Freire, P., Lyne, A. G., Manchester, R. N., 2000, ApJ, 535,975

Carrigan, R. A., Jr., 2009, ApJ, 698, 2075

Chennamangalam, J., Lorimer, D. R., 2014, MNRAS Letters, in press, arXiv: 1311.4846

Chennamangalam, J., Lorimer, D. R., Mandel, I., Bagchi, M., 2013, MNRAS, 431, 874

Chennamangalam, J., Siemion, A. P. V., Lorimer, D. R., Werthimer, D., 2013, arXiv:1311.4608

Cohen, R. J., Downs, G., Emerson, R., Grimm, M., Gulkis, S., Stevens, G., Tarter, J., 1987, MNRAS, 225, 491

Colgate, S. A., Noerdlinger, P. D., 1971, ApJ, 165, 509

Corbet, R. H. D., 1997, JBIS, 50, 253 
Cordes, J. M., 1993, in Shostak, G. S., ed., ASP Conf. Ser. Vol. 47, Third Decennial US-USSR Conference on SETI, Astron. Soc. Pac., San Fracisco, p. 257

Cordes, J. M., 2002, in Stanimirovic, S., Altschuler, D., Goldsmith, P., Salter, C., ASP Conf. Ser. Vol 278, Single-Dish Radio Astronomy: Techniques and Applications, Astron. Soc. Pac., San Francisco, p. 227

Cordes J. M., Lazio T. J. W., 1997, ApJ, 475, 557

Cordes, J. M., Lazio, T. J. W., 2002, arXiv:astro-ph/0207156

Cordes, J. M., McLaughlin, M. A., 2003, ApJ, 596, 1142

Cordes, J. M., Sullivan, W. T., III, 1995, in Shostak, G. S., ed., ASP Conf. Ser. Vol. 74, Progress in the Search for Extraterrestrial Life, Astron. Soc. Pac., San Francisco, p. 325

Cunningham, M. R., et al., 2007, MNRAS, 376, 1201

Demorest, P. B., Pennucci, T., Ransom, S. M., Roberts, M. S. E., Hessels, J. W. T., 2010, Nature, 467, 1081

Deneva, I. S., 2010, Ph.D. thesis, Cornell University

Deneva, J. S., Cordes, J. M., Lazio, T. J. W., 2009, ApJ, 702, L177

Dexter, J., O’Leary, R. M., 2013, arXiv:1310.7022

Dyson, F. J., 1960, Science, 131, 1667 
Eatough, R. P., Kramer, M., Klein, B., Karuppusamy, R., Champion, D. J., Freire, P. C. C., Wex, N., Liu, K., 2013, IAUS, 291, 382

Eatough, R. P., et al., 2013, ATel \#5040

Eatough, R. P., et al., 2013, Nature, 501, 391

Ehrenfreund, P., Charnley, S. B., 2000, ARA\&A, 38, 427

Ellis, G. R. A., McCulloch, P. M., 1967, Australian J. Phys., 20, 583

Fabian, A. C., 1977, JBIS, 30, 112

Falcke, H., Rezzolla, L., 2013, A\&A, in press, arXiv:1307.1409

Faucher-Giguère, C.-A., Kaspi, V. M., 2006, ApJ, 643, 332

Figer, D. F., Rich, R. M., Kim, S. S., Morris, M., Serabyn, E., 2004, ApJ, 601, 319

Fruchter, A. S., Goss, W. M., 1990, ApJ, 365, L63

Fruchter, A. S., Goss, W. M., 2000, ApJ, 536, 865

Genzel, R., Eisenhauer, F., Gillessen, S., Rev. Modern Phys., 82, 3121

Ginzburg, V. L., Zheleznyakov, V. V., 1975, ARA\&A, 13, 511

Gold, T., 1968, Nature, 218, 731

Golden, A., Shearer, A., 1999, A\&A, 342, L5

Goldreich, P., Julian, W. H., 1969, ApJ, 157, 869 
Gregory, P. C., 2005, Bayesian Logical Data Analysis for the Physical Sciences: A Comparative Approach with Mathematica Support. Cambridge University Press, Cambridge, UK

Grindlay, J. E., Camilo, F., Heinke, C. O., Edmonds, P. D., Cohn, H., Lugger, P., 2002, ApJ, 581, 470

Hessels, J. W. T., Ransom, S. M., Stairs, I. H., Freire, P. C. C., Kaspi, V. M., Camilo, F., 2006, Science, 311, 1901

Hessels, J. W. T., Ransom, S. M., Stairs, I. H., Kaspi, V. M., Freire, P. C. C., 2007, ApJ, 670, 363

Hewish, A., Bell, S. J., Pilkington, J. D. H., Scott, P. F., Collins, R. A., 1968, Nature, 217, 709

Horowitz, P., Sagan, C., 1993, ApJ, 415, 218

Howard, A., et al., 2007, Acta Astronautica, 61, 78

Johnston, S., Kramer, M., Lorimer, D. R., Lyne, A. G., McLaughlin, M., Klein, B., Manchester, R. N., 2006, MNRAS, 373, L6

Jones, P. A., Cunningham, M. R., Godfrey P. D., Cragg, D. M., 2007, MNRAS, 374,579

Jørgensen, J. K., Favre, C., Bisschop, S. .E., Bourke, T. L., van Dishoeck, E. F., Schmalzl, M., 2012, ApJ, 757, L4 
Kardashev, N. S., 1964, SvA, 8, 217

Kennea, J. A., et al., 2013, ApJ, 770, L24

Kijak, J., Gil, J., 2003, A\&A, 397, 969

Kocsis, B., Ray, A., Portegies Zwart, S., 2012, ApJ, 752, 67

Kramer, M., Klein, B., Lorimer, D., Müller, P., Jessner, A., Wielebinski, R., 2000, ASPC, 202, 37

Kramer, M., Xilouris, K. M., Lorimer, D. R., Doroshenko, O., Jessner, A., Wielebinski, R., Wolszczan, A., Camilo, F., 1998, ApJ, 501, 270

Kuan, Y.-J., Charnley, S. B., Huang H.-C., Tseng W.-L., Kisiel, Z., 2003, ApJ, 593, 848

Kulkarni, S. R., Narayan, R., Romani, R. W., 1990, ApJ, 356, 174

Kulkarni, S. R., Goss, W. M., Wolszczan, A., Middleditch, J., 1990, ApJ, 363, L5

Lang, K. R., Bookbinder, J., Golub, L., Davis, M. M., 1983, ApJ, 272, L15

Lattimer, J. M., Prakash, M., 2001, ApJ, 550, 426

Law, C. J., Yusef-Zadeh, F., Cotton, W. D., Maddalena, R. J., 2008, ApJS, 177, 255

Learned, J. G., Kudritzki, R.-P., Pakvasa, S., Zee, A., 2008, arXiv:0809.0339

Levin, L. et al., 2010, ApJ, 721, L33 
Liu, K., Wex, N., Kramer, M., Cordes, J. M., Lazio, T. J. W., 2012, ApJ, 747, 1

Loeb, A., Shvartzvald, Y., Maoz, D., 2014, MNRAS Letters, doi:10.1093/mnrasl/slt177

Lorimer, D. R., Kramer, M., 2005, Handbook of Pulsar Astronomy, Cambridge Univ. Press, Cambridge, UK

Lorimer, D. R., Yates, J. A., Lyne, A. G., Gould, D. M., 1995, MNRAS, 273, 411

Lorimer, D. R., Bailes, M., McLaughlin, M. A., Narkevic, D. J., Crawford, F., 2007, Science, 318,777

Macquart, J.-P., Kanekar, N., Frail, D. A., Ransom, S. M., 2010, ApJ, 715, 939

Magro, A., Hickish, J., Zarb Adami, K., 2013, arXiv:astro-ph/1307.5700

Magro, A., Karastergiou, A., Salvini, S., Mort, B., Dulwich, F., Zarb Adami, K., 2011, MNRAS, 417, 2642

Manchester, R. N., Hobbs, G. B., Teoh, A., Hobbs, M., 2005, AJ, 129, 1993

Manchester R. N., et al., 2001, MNRAS, 328, 17

McConnell, D., Deshpande, A. A., Connors, T., Ables, J. G., 2004, MNRAS, 348, 1409

McLaughlin, M. A., et al., 2006, Nature, 439, 817

Meylan, G., Heggie, D. C., 1997, A\&AR, 8, 1 
Mori, K., et al., 2013, ApJ, 770, L23

Morris, D. J., et al., 2002, MNRAS, 335, 275

Narayan, R., 1992, Philos. Trans. R. Soc. Lond. A, 341, 151

Ortolani, S., Barbuy, B., Bica, E., Zoccali, M., Renzini, A., 2007, A\&A, 470, 1043

Pfahl, E., Loeb, A., 2004, ApJ, 615, 253

Popov, S. B., Postnov, K. A. 2013, arXiv:1307.4924

Purvinis, G. M., Maldonado, T. A., 2010, in Bass, M., DeCusatis, C. M., Enoch, J. M., Lakshminarayanan, V., Li, G., MacDonald, C., Mahajan, V. N., Stryland, E. V., eds., Handbook of Optics, Third Edition Vol. V, Atmospheric Optics, Modulators, Fiber Optics, X-Ray and Neutron Optics, McGraw-Hill

Ransom, S. M., Hessels, J. W. T., Stairs, I. H., Freire, P. C. C., Camilo, F., Kaspi, V. M., Kaplan, D. L., 2005, Science, 307, 892

Ransom, S. M., Demorest, P., Ford, J., McCullough, R., Ray, J., DuPlain, R., Brandt, P., 2009, AAS Meeting, 214, 605.08

Rea, N. et al., 2013, ApJ, 775, L34

Redman, S. L., Rankin, J. M., 2009, MNRAS, 395, 1529

Rees, M. J., 1977, Nature, 266, 333

Rickett, B. J., 1990, ARA\&A, 28, 561 
Ridley, J. P., Lorimer, D. R., 2010, MNRAS, 404, 1081

Ridley, J. P., Crawford, F., Lorimer, D. R., Bailey, S. R., Madden, J. H., Anella, R., Chennamangalam, J., 2013, MNRAS, 433, 138

Roshi, D. A., et al., 2011, General Assembly and Scientific Symposium, 2011 XXXth URSI, doi:10.1109/URSIGASS.2011.6051280

Servillat, M., Heinke, C. O., Ho, W. C. G., Grindlay, J. E., Hong, J., van den Berg, M., Bogdanov, S., 2012, MNRAS, 423, 1556

Shannon, R. M., Johnston, S., 2013, MNRAS, 435, L29

Siemion, A. P. V., et al., 2013, ApJ, 767, 94

Siemion, A., et al., 2013, IAUS, 291, 57

Snyder, L. E., et al., 2005, ApJ, 619, 914

Sofue, Y., 1994, ApJ, 431, L91

Spitler, L. G., et al., 2014, ApJ, 780, L3

Sullivan, W. T., III, Cordes, J. M., 1995, in Shostak, G. S., ed., Progress in the Search for Extraterrestrial Life, ASP Conf. Ser., Vol. 74, Astron. Soc. Pac., San Francisco, 337

Tauris, T. M., Manchester, R. N., 1998, MNRAS, 298, 625

Thornton, D., et al., 2013, Science, 341, 53 
Totani, T., 2013, arXiv:1307.4985

Turk, P. J., Lorimer, D. R., 2013, MNRAS, 436, 3720

Wall, J. V., Jenkins, C. R., 2003, Practical Statistics for Astronomers, Cambridge Univ. Press, Cambridge, UK

Wang, N., Manchester, R. N., Johnston, S., 2007, MNRAS, 377, 1383

Wharton, R. S., Chatterjee, S., Cordes, J. M., Deneva, J. S., Lazio, T. J. W., 2012, ApJ, 753, 108

Woodley, K. A., et al., 2012, AJ, 143, 50

Young, M. D., Manchester, R. N., Johnston, S., 1999, Nature, 400, 848

Zepf, S. E., 2003, in Engvold, O., ed, Highlights of Astronomy, Vol. 13, as presented at the XXVth General Assembly of the IAU 2003, Astron. Soc. Pac., San Francisco, p. 347

Zhang, B., Hong, B. H., Qiao, G. J., 1999, ApJ, 514, L111 